\title{
WestVirginiaUniversity
}

THE RESEARCH REPOSITORY @ WVU

Graduate Theses, Dissertations, and Problem Reports

2007

\section{Color space analysis for iris recognition}

\author{
Matthew K. Monaco \\ West Virginia University
}

Follow this and additional works at: https://researchrepository.wvu.edu/etd

\section{Recommended Citation}

Monaco, Matthew K., "Color space analysis for iris recognition" (2007). Graduate Theses, Dissertations, and Problem Reports. 1859.

https://researchrepository.wvu.edu/etd/1859

This Thesis is protected by copyright and/or related rights. It has been brought to you by the The Research Repository @ WVU with permission from the rights-holder(s). You are free to use this Thesis in any way that is permitted by the copyright and related rights legislation that applies to your use. For other uses you must obtain permission from the rights-holder(s) directly, unless additional rights are indicated by a Creative Commons license in the record and/ or on the work itself. This Thesis has been accepted for inclusion in WVU Graduate Theses, Dissertations, and Problem Reports collection by an authorized administrator of The Research Repository @ WVU. For more information, please contact researchrepository@mail.wvu.edu. 


\title{
Color Space Analysis for Iris Recognition
}

\author{
by \\ Matthew K. Monaco \\ Thesis submitted to the \\ College of Engineering and Mineral Resources \\ at West Virginia University \\ in partial fulfillment of the requirements \\ for the degree of \\ Master of Science \\ in \\ Electrical Engineering
}

Arun A. Ross, Ph.D., Chair

Lawrence Hornak, Ph.D.

Xin Li, Ph.D.

Lane Department of Computer Science and Electrical Engineering

Morgantown, West Virginia

2007

Keywords: Iris, Iris Recognition, Multispectral, Iris Anatomy, Iris Feature Extraction, Iris Fusion, Score Level Fusion, Multimodal Biometrics, Color Space Analysis, Iris Enhancement, Iris Color Analysis, Color Iris Recognition

Copyright 2007 Matthew K. Monaco 


\author{
Abstract \\ Color Space Analysis for Iris Recognition \\ by \\ Matthew K. Monaco \\ Master of Science in Electrical Engineering \\ West Virginia University
}

Arun A. Ross, Ph.D., Chair

This thesis investigates issues related to the processing of multispectral and color infrared images of the iris. When utilizing the color bands of the electromagnetic spectrum, the eye color and the components of texture (luminosity and chromaticity) must be considered. This work examines the effect of eye color on texture-based iris recognition in both the near-IR and visible bands. A novel score level fusion algorithm for multispectral iris recognition is presented in this regard. The fusion algorithm - based on evidence that matching performance of a texture-based encoding scheme is impacted by the quality of texture within the original image - ranks the spectral bands of the image based on texture quality and designs a fusion rule based on these rankings. Color space analysis, to determine an optimal representation scheme, is also examined in this thesis. Color images are transformed from the sRGB color space to the CIE Lab, YCbCr, CMYK and HSV color spaces prior to encoding and matching. Also, enhancement methods to increase the contrast of the texture within the iris, without altering the chromaticity of the image, are discussed. Finally, cross-band matching is performed to illustrate the correlation between eye color and specific bands of the color image. 
I dedicate this work to my friends and family. 


\section{Acknowledgments}

Without the help of those around me I would not of been able to accomplish so much. I would like to take a moment to thank those who have helped and inspired me throughout this journey.

I must first thank my committee chair Dr Arun Ross, without his guidance and monitoring I would not be where I am today. Without his dedication and insight much of this work would not of been possible. It has been an honor to have him as a advisor, teacher and friend through out my graduate education. I must acknowledge Dr Larry Hornak and Dr Xin Li, also members of my committee, for their contributions and advice during my time as a graduate student.

It is important that my fellow students in the lab know that their help and friendship over the last year and a half was greatly appreciated. I truly enjoyed the time I spent with all of you: Chris, Nikhil, Phani, Rajiv, Rohan, Simona, Samir, Shigefumi, Raghav and Manisha. I hope you enjoyed your time with me as much as I did with you. I hope you all the best and good luck.

Lastly and most importantly I would like to thank my friends and family for their support and motivation. Without the constant encouragement and patience of my parents, Nick, Mike and especially Abby you would not be reading this today. 


\section{Contents}

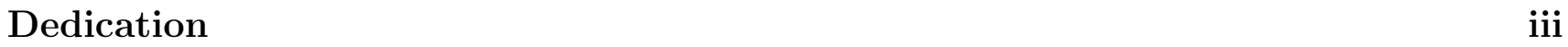

Acknowledgments $\quad$ iv

List of Figures vii

List of Tables $\quad \mathrm{x}$

1 Introduction 1

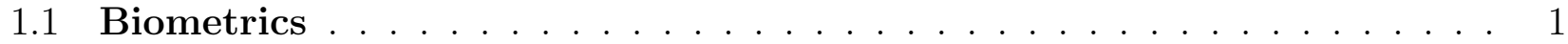

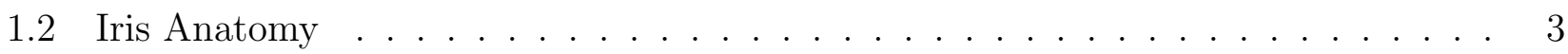

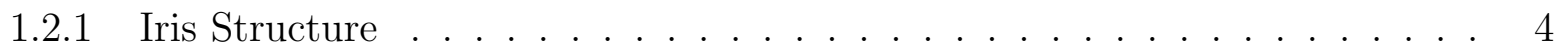

1.2 .2 Iris Pigmentation . . . . . . . . . . . . . . . . 6

1.3 Iris Recognition . . . . . . . . . . . . . . . . . . . 7

1.4 Multibiometrics . . . . . . . . . . . . . . . . . . . . . 9

1.4.1 Multibiometric Fusion . . . . . . . . . . . . . . . . . 11

1.5 Multispectral Iris Recognition . . . . . . . . . . . . . . . . . . . . 12

1.5.1 Multispectral Imaging . . . . . . . . . . . . . . . . . . . . . 12

1.5.2 Multispectral Iris Imaging . . . . . . . . . . . . . . . . . . . 13

1.5.3 Multispectral Image Data . . . . . . . . . . . . . . . . . . . 15

1.5.4 Color (RGB) Iris Database . . . . . . . . . . . . . . . . . . 16

1.6 Color Space Analysis . . . . . . . . . . . . . . . . . . . . . . . . . . . . . . . 17

1.6.1 Color Model . . . . . . . . . . . . . . . . . . . . . . . . . . . . 18

1.6 .2 Color Space . . . . . . . . . . . . . . . . . . . . . . . . . . 22

1.7 Literature Review . . . . . . . . . . . . . . . . . . . . . . . . . . 23

1.7.1 Multispectral Iris Recognition Analysis . . . . . . . . . . . . . . . 23

1.7.2 Fake Iris Detection through Variations of Reflectance Ratios . . . . . . . . 25

1.8 Contributions of this Thesis . . . . . . . . . . . . . . . 25

2 Dynamic Score Level Fusion $\quad 27$

2.1 Motivation . . . . . . . . . . . . . . . . . . . . 27 


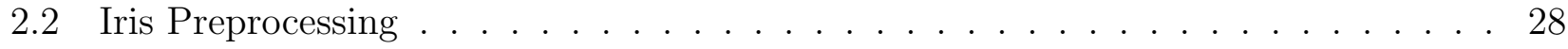

2.3 Segmentation . . . . . . . . . . . . . . . . . . 32

2.4 Encoding and Test for Statistical Independence . . . . . . . . . . . . . . 36

2.5 Score Level Fusion . . . . . . . . . . . . . . . . . . . . . . . . . 39

2.5.1 Dynamic Score Level Fusion Results for Gabor Based Texture Encoding . . 44

2.6 Local Binary Patterns _ . . . . . . . . . . . . . . . . . . . . . . . . 48

2.6.1 Dynamic Score Level Fusion Based on Local Binary Pattern Matching Scores 50

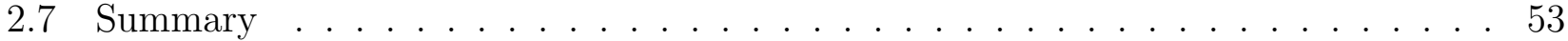

3 Iris Color and Color Space Analysis $\quad 54$

3.1 Motivation . . . . . . . . . . . . . . . . . . . . 54

3.2 Color Space Transformations . . . . . . . . . . . . . . . . . . . 55

3.3 Image Processing and Color Space Adaption . . . . . . . . . . . . . . . . . 60

3.4 Texture Enhancement _. . . . . . . . . . . . . . . . . . . . . . 60

3.4.1 Textural Quality in Enhanced Iris Templates . . . . . . . . . . . . . . 61

3.5 Iris Recognition in Alternative Color Spaces . . . . . . . . . . . . . . . . . 67

3.5.1 Cross-Band Correlation . . . . . . . . . . . . . . . . . . 75

3.5.2 sRGB Cross-Band Matching . . . . . . . . . . . . . . . . 77

3.6 Discussion and Summary . . . . . . . . . . . . . . . . . . . 77

4 Summary and Future Work 81

4.1 Summary of Work . . . . . . . . . . . . . . . . . . . . . 81

4.2 Future Work . . . . . . . . . . . . . . . . . . . . . . . . . . . 84

$\begin{array}{lr}\text { References } & 86\end{array}$

$\begin{array}{ll}\text { A Appendix } & 91\end{array}$

A.1 Multispectral Iris Data Collection Consent Form . . . . . . . . . . . . . . . . . 91 


\section{List of Figures}

1.1 Flowchart depicting the various stages of a traditional biometric system . . . . . . 3

1.2 Dissection of a human eye showing the structures of the eye. . . . . . . . . . . 5

1.3 Major regions of the visible iris. . . . . . . . . . . . . . . . 6

1.4 An image of an eye captured in near-IR, common to most commercial iris recognition systems. In this case, the specular reflection has been confined to the pupil which ensures that no iris textural information is lost. . . . . . . . . . . 8

1.5 Graphical representation of a binary IrisCode ${ }^{\circledR}$. The hamming distance between two IrisCodes ${ }^{\circledR}$ is used to determine the matching score. . . . . . . . . . . . . . . 9

1.6 False color image and the four band channels which are extracted from it. . . . . . 13

1.7 The normalized transmittance band-pass wavelengths for each prism and sensor of the multispectral device. Portions of the graph which are filled represent the combined response of the sensors and prisms. . . . . . . . . . . . . . 15

1.8 Demonstration of the multispectral camera capturing an image of a subject's eye. 16

1.9 Visual comparison of the color RGB image (left) and the CIR image (right). . . . 17

1.10 Typical examples of color images found within the UBIRIS database. . . . . . . . 17

1.11 Individual components of a sRGB image. . . . . . . . . . . . . . . . . 18

1.12 Individual components of a CIE Lab image. . . . . . . . . . . . . . . . . . . 19

1.13 Individual components of a YCbCr image. . . . . . . . . . . . . . . . . . . . 20

1.14 Individual components of a HSV image. . . . . . . . . . . . . . . . . . . . 21

1.15 Visual representation of the hue scale showing the corresponding color value. . . 21

1.16 Individual components of a CMYK image. . . . . . . . . . . . . . . . . 22

1.17 The gamut of all representable colors in the sRGB color space. . . . . . . . . . . . 23

1.18 3D gamut of the CIR XYZ color space. The third dimension allows for the inclusion of brightness into the gamut. . . . . . . . . . . . . . . . 24

2.1 An iris image before (left) and after (right) textural enhancement using Lab histogram equalization. . . . . . . . . . . . . . . . . 28

2.2 Steps to isolate the specular reflection caused by the fiber optic ring light. . . . . . 30

2.3 The original (left) image which includes specular reflection compared to the final impainting results (right). . . . . . . . . . . . . . . . 31 
2.4 Binary iris image after pupil thresholding. . . . . . . . . . . . . . . . 33

2.5 Segmented Pupil . . . . . . . . . . . . . . . . . . . . . 33

2.6 Localization of the regions of ROI within in the iris. . . . . . . . . . . . 35

2.7 Once the image is segmented it is unwrapped and normalized into a fixed sized

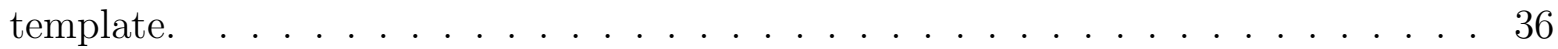

2.8 Three dimensional plots of the real (left) and imaginary (right) parts of the Gabor filter. . . . . . . . . . . . . . . . . . . . . 38

2.9 Matching performance using the Gabor based encoding scheme. The ROC curve shows the performance of each channel independently when compared against all other images from the same channel across the whole multispectral database.

2.10 Comparing the imaging qualities of the four channel images for the three classes of eye colors. Based on the eye color, levels of information are present in each channel. Starting from the top right and going clockwise the channels are red, green, blue and IR. . . . . . . . . . . . . . . . .

2.11 Four channels of a multispectral normalized blue iris image. For all measurements except uniformity as the amount/quality of texture increases in the image the score also increases. On the contrary, an image with high levels of texture will have a low uniformity score. . . . . . . . . . . . . . . . . . . . 43

2.12 Performance curves for eye color class when the dynamic fusion rule is applied to only users of each color class independently.

2.13 Matching results of the dynamic fusion algorithm versus the baseline fusion algorithm and the individual channel performance when applied to the multispectral image database. . . . . . . . . . . . . . . . . . . . . 4 46

2.14 Dynamic score level fusion performance on color images. . . . . . . . . . . . . . 47

2.15 Graphical representation of a 11x11 LBP neighborhood. The pixels which are colored blue are the only pixels within the neighborhood that are considered. . . .

2.16 The ROC curves demonstrating the single-band matching performance as well as the default fusion rule and dynamic fusion rule. Users are classified by eye color to emphasize the role color has on matching performance in the visible spectrum.

2.17 ROC curve for the entire database encoded using LBP. Performance curves are shown for each band of the image as well as the baseline and dynamic fusion

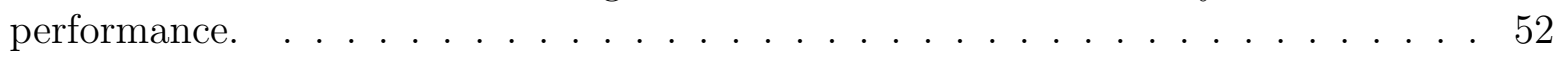

3.1 Comparison of contrast enhancement techniques on a segmented iris image. . . . . 62

3.2 Matching performance of images enhanced using HSV histogram equalization. . . 64

3.3 Matching performance of images enhanced using CIE Lab histogram equalization. 65

3.4 Matching performance of images enhanced using $\mathrm{YCbCr}$ histogram equalization. . 66

3.5 Matching performance in the sRGB color space. . . . . . . . . . . . . . . . 69

3.6 Matching performance in the HSV color space. . . . . . . . . . . . . . . . . . 70

3.7 Matching performance in the $\mathrm{YCbCr}$ color space. . . . . . . . . . . . . . . 71

3.8 Matching performance in the CIE Lab color space. . . . . . . . . . . . . . . . 72 
3.9 Matching performance in the CMYK color space. . . . . . . . . . . . . . . . 73

3.10 Matching performance of cross-band comparison of sRGB image bands. . . . . . . 78 


\section{List of Tables}

2.1 Measures of image texture used by the dynamic fusion algorithm. Let $z_{i}$ be the location of a single pixel in the Cartesian plane of an image. . . . . . . . . . . 42

2.2 The EER for dynamic fusion when using the Gabor based encoding scheme. . . . 44

2.3 The EER for Dynamic fusion when using Gabor based encoding scheme on color iris images. . . . . . . . . . . . . . . . . . . . 4 46

2.4 The EER for Dynamic fusion when using LBP based encoding scheme. . . . . . . 52

3.1 Measurement index for contrast within the iris template. For all indices except uniformity a higher score represents greater amounts of texture. . . . . . . . . 6 63

3.2 The EER for the images enhanced via HSV histogram equalization. . . . . . . . . 63

3.3 The EER for the images enhanced via CIE Lab histogram equalization. . . . . . . . 63

3.4 The EER for the images enhanced via $\mathrm{YCbCr}$ histogram equalization. . . . . . . . 67

3.5 The EER for the sRGB color space. . . . . . . . . . . . . . . . . 68

3.6 The EER for the HSV color space. . . . . . . . . . . . . . . . . . 68

3.7 The EER for the $\mathrm{YCbCr}$ color space. . . . . . . . . . . . . . . . . . . 74

3.8 The EER for the CIE Lab color space. . . . . . . . . . . . . . . . . . . . . 74

3.9 The EER for the CMYK color space. . . . . . . . . . . . . . . . . . 74

3.10 Matching score cross-band correlation in the sRGB color space. . . . . . . . . . 75

3.11 Matching score cross-band correlation in the HSV color space. . . . . . . . . . . . 75

3.12 Matching score cross-band correlation in the $\mathrm{YCbCr}$ color space. . . . . . . . . . . 75

3.13 Matching score cross-band correlation in the CIE Lab color space. . . . . . . . . . 76

3.14 Matching score cross-band correlation in the CMYK color space. . . . . . . . . . . 76

3.15 The EER for cross-band matching of sRGB image bands. . . . . . . . . . . . . 77 


\section{Chapter 1}

\section{Introduction}

\subsection{Biometrics}

Derived from the Greek words "bios" meaning life and "metron" meaning measure, biometrics is defined as the method to uniquely recognize a human based on either physical or behavioral characteristics $[1,2]$. Biometric systems provide verification ("Is this person who they claim to be?") and identification ("Who is this person?") functions by analyzing "who a person is" (biometric characteristic) rather than by "what a person has or remembers" (smartcard, password, etc.). Though any physical or behavioral characteristics of an individual can be considered a biometric, an ideal biometric must exhibit the following attributes [3]:

- Universality: How common the biometric is throughout the entire population.

- Uniqueness: How well the biometric can distinguish one individual from another.

- Permanence: Does the biometric change with time (aging)?

- Collectability: How easy it is to acquire the biometric.

- Performance: Indications of how accurate, fast and robust the biometric is.

- Acceptability: How well the biometric is accepted by the end-users. 
- Circumvention: How resistant the biometric is against fooling or "spoofing".

The most common biometrics implemented today, which have the afore mentioned properties include Iris, Fingerprint, Face and Hand Geometry [4]. It should be noted that these are examples of physical biometrics while Gait, Keystroke and Signature are all examples of behavioral biometrics.

Typically, biometric systems are designed having five modules (Figure 1.1). The first module is the sensor where the biometric trait is extracted from the user. For most biometrics (iris, fingerprint, face, etc.) the capture device is a camera. The camera can range from a near-IR imaging sensor [5], to a RGB sensor [6], to a thermal sensor [7]. Other acquisition devices include microphone (voice recognition [8]) and chemical sensors (fingerprint [9]), just to name a few. The second module is the segmentation stage. Segmentation involves the removal of information from the data captured by the sensoring device which does not pertain to the measurable trait. For an iris image, this involves the removal of the eyelashes, sclera and eyelid. The third module is the feature extraction module that converts the segmented entity into a numerical feature set. In an iris recognition system, algorithms based on Gabor filters [10-12] are used to encode the information within the segmented iris image to create a template used by the fourth segment of the biometric system-matching. The matcher compares the extracted template against other templates to give a quantitative assessment of likeness. The match score may be a "similarity" score or a "distance" score. Two templates from the same subject are likely to have a high similarity score but a low distance score. Measures of distance, such as the Hamming distance, are commonly used for iris recognition. The final stage of the biometric system is the decisionmaking module. In this module, the match score output by the previous stage is used to render the final decision about the identity of the subject. Typically, a threshold is employed in order to render this decision. The threshold can vary depending on the application. If the objective of the biometric system is high security, than the threshold should move closer to the maximum potential (similarity) score. As the score moves in this direction, the possibility of false rejects increases thereby making the system more difficult to use. If the objective of the system is to 
provide an easy, accessible system, the threshold should be moved away from the maximum possible similarity score, thus making the system more accessible, but more likely to allow false accepts.

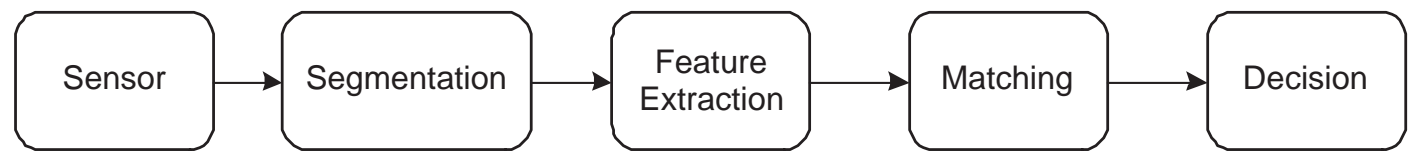

Figure 1.1: Flowchart depicting the various stages of a traditional biometric system

\subsection{Iris Anatomy}

The iris is responsible for regulating the amount of light that enters the pupil and is absorbed by the retina. The amount of light that enters the pupil is regulated through the constriction or dilation of the pupil. Since the pupil has no mechanism to regulate it's shape on its own the iris, through the contraction of muscles, provides the required movement. The process of mydriasis is the dilation of the pupil by the dilator pupillae muscle which is triggered by activity in the parasympathetic nerve in low lighting conditions. On the contrary, in the presence of intense light the pupil will contract. The contraction is caused by a process called miosis where the constrictor pupillae muscle, triggered by the parasympathetic nerve, reduces the amount of light which enters the pupil.

The iris is the only internal human organ which is visible from the outside of the body allowing it to be easily imaged. The ease of imaging makes iris an ideal biometric. The iris is formed prenatally, independent of genetic genotype. The process is considered to be random, unique, chaotic and only dependent on initial conditions in the embryonic mesoderm [13]. Due to the chaotic and random nature of the iris and the ability to easily acquire a contact-less image, the iris is considered to be a strong biometric. 


\subsubsection{Iris Structure}

Due to the vibrant color and texture of the iris it is typically the most visible and distinguishable part of the human eye. The average diameter of the iris is approximately $12 \mathrm{~mm}$ with an average thickness around .5mm [14]. The iris is thickest at the collarette and continues to thin out radially as you move away from the pupil. The truncated cone shape of the iris is due to the anterior surface of the lens pressing against the posterior iris.

Irides are composed of fibrovascular tissue known as stroma. A stroma connects the sphincter pupillae, which contracts the pupil, and the dilator pupillae, responsible for dilating the pupil. The dilator pupillae, a dilator muscle, and the sphincter pupillae, a sphincter muscle, are a group of muscles which are found in irides of all vertebrates. The back surface of the iris is covered by the iris pigment epithelium. The surface is covered by an epithelial layer which is two cells thick and results in the color of the iris. The front (outside) surface of the iris does not consist of a epithelium layer. The root of the iris is the outer edge of the iris and attaches the sclera to the anterior ciliary body of the iris. The sclera is the opaque (typically white) fibrous, protective layer of the eye which comprises majority of the iris surface. The ciliary body, together with the iris, known as the anterior uvea, is the circumferential tissue inside the eye (Figure 1.2) ${ }^{1}$. The ciliary body is mostly responsible for providing most of the nutrients to the lens and cornea as well as conducting waste management for the same areas. The region of the iris in front of the root is responsible for drainage of aqueous humour, crucial to the maintenance of the eye. Aqueous humour is a thick watery substance responsible for inflating the globe of the eye and providing nutrition for the avascular ocular tissues.

The iris is divided into two major regions (Figure 1.3): pupillary zone and ciliary zone. The pupillary zone is the inner region of the iris which ends at the boundary of the pupil. The ciliary zone extends from its origin to the ciliary body. The collarette is the region where the sphincter muscle and dilator muscle overlap. This is considered the point at which the pupillary portion and ciliary portion interchange. Iris color as well as structural features often vary between the two

\footnotetext{
${ }^{1}$ www.bartleby.com/107/225.html
} 


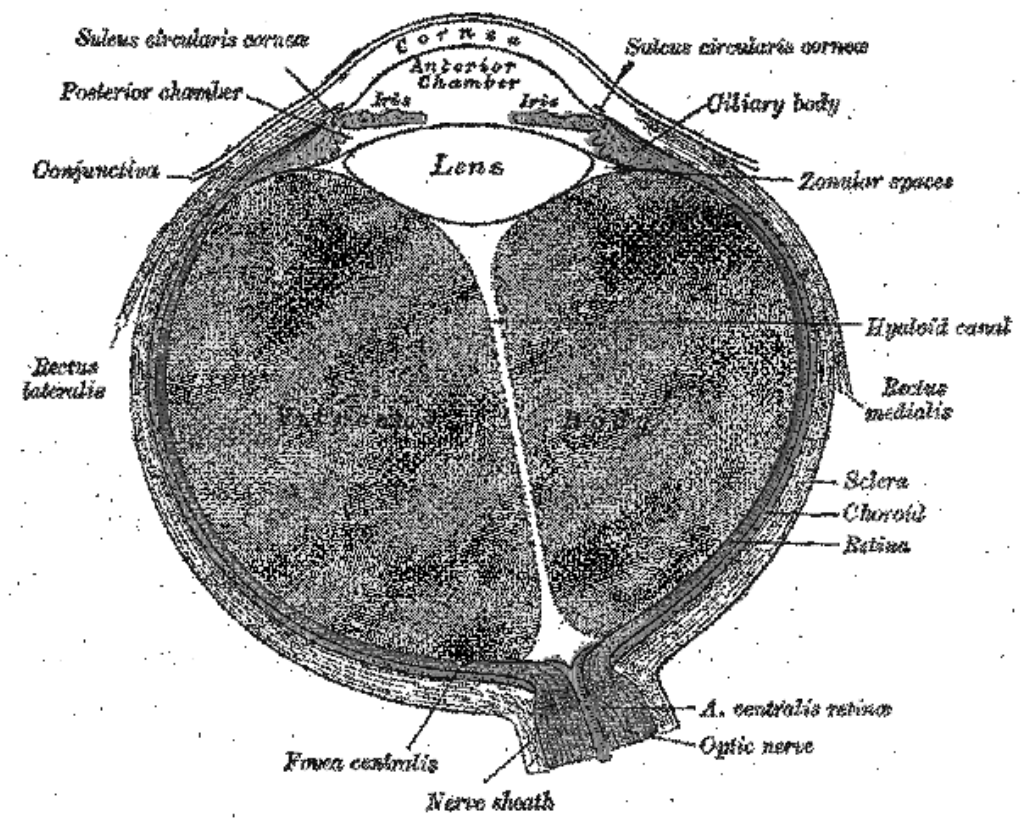

Figure 1.2: Dissection of a human eye showing the structures of the eye. 
zones. Fruch's crypts are openings which appear on either side of the collarette and allows stroma and other deep iris tissue to be submerged in aqueous humor during dilation and contraction of the pupil. Though these features are common on both portions of the outer iris they are much more common in the ciliary zone of the iris.

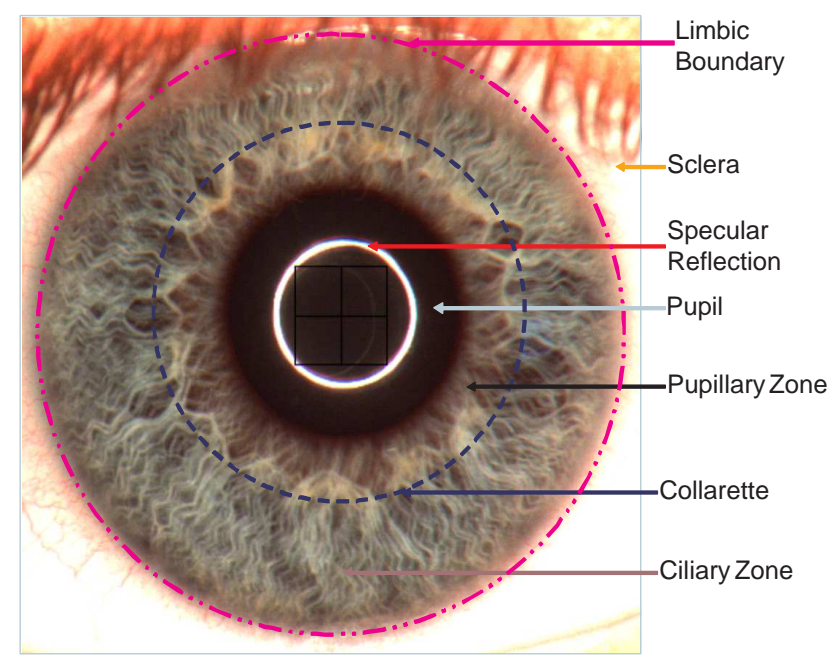

Figure 1.3: Major regions of the visible iris.

The iris is comprised of six layers starting from the anterior(front) to the posterior(back). The layers are as follows: anterior border layer, stroma of iris, iris sphincter muscle, iris dilator muscle, anterior pigment myoepithelium and the posterior pigment epithelium. The anterior portion of the iris is the focus of most recognition systems due to accessability for imaging.

\subsubsection{Iris Pigmentation}

It is common to see a wide variety of strongly pigmented color within an iris. Colors such as brown, blue, green, grey, and hazel are found in irides. In rare cases, a lack of pigmentation can lead to a pinkish-white iris color resulting from oculo-cutaneous albinism [15]. Oculo-cutaneous albinism is a result of obstruction of pigment due to blood vessels. The pigment of an iris is the result of the molecule melanin. In the stromal pigment cell melanin is a yellowish-brown to dark 
brown, and in the iris pigment epithelium it is black. Subcellular bundles called melanosomes contain varying degrees of melanin dispersion. The overall dispersion of melanin has only a minor influence on the color of the iris. Due to unbalanced dispersion of melanin it is common for a two-tone pigmentation to arise in the iris. A resulting effect is the collection of brownish stromal melanin in the thin anterior body of the iris. Similar to the pigments responsible for skin and hair color, melanin of the iris is a polymer of one or two monomer molecules [16].

The perception of iris color is a complex phenomenon combining the effects of pigmentation, texture, fibrous tissue and blood vessels within the iris stroma. Iris color besides brown and black are due to selective reflection and absorption from other stromal components. The most prevalent forms of reflection and absorption are a result of hemoglobin in the blood vessels, collagen in the vessel walls and the stroma itself. Other less common elements such as Rayleigh scattering, Tyndall scattering, and diffraction are also responsible for the color of the iris. Shorter wavelengths of light (blue light) are commonly reflected or scattered by the iris stroma, while the longer wavelengths are typically absorbed or penetrate the iris. Rarely, in aged or green eyes, a yellowish pigment known as lipofuscin may be present. It is common for Caucasian newborns to be born with grey or blue irides. From birth, exposure to light triggers the production of melanin in the iris. By age three, the melanin content is high enough that the iris takes on its natural shade of green, hazel, brown or amber. Past the age of three, changes in iris color are commonly due to exposure to sunlight. In a similar manner to the skin, as the iris is exposed to sunlight an increase of melanin occurs. It is not common for green irides to become hazel, or light brown irides to become dark brown, in the summer [17].

\subsection{Iris Recognition}

Commercial iris recognition devices image high resolution images of the eye in the near-IR domain. Imaging in the near-IR domain allows for non-intrusive illumination which reduces specular reflection from the convex cornea as well as provides illumination without causing physical 
discomfort to the user (Figure 1.4). Analysis of the imaging spectrum in commercial iris recognition systems show that two popular brands, Panasonic and Oki, use illumination which peak at $850 \mathrm{~nm}$ with a relatively narrow band pass. Systems developed by LG image in the range of 750 $\mathrm{nm}$ with multiple wavelength illumination [18]. The texture observed under near-IR illumination is the traditional biometric indicator used in commercial iris recognition systems.

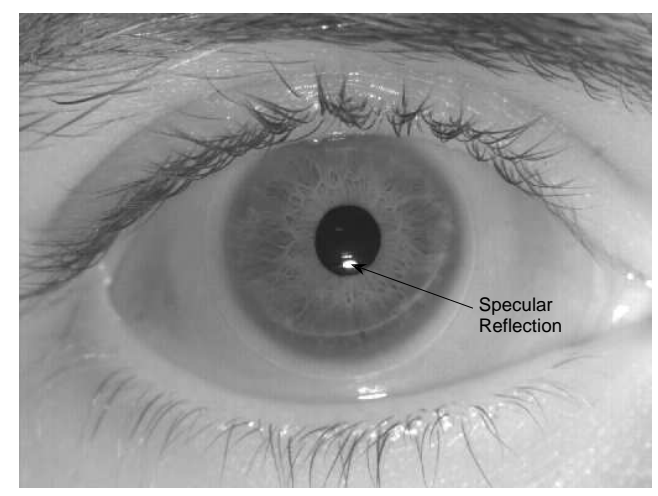

Figure 1.4: An image of an eye captured in near-IR, common to most commercial iris recognition systems. In this case, the specular reflection has been confined to the pupil which ensures that no iris textural information is lost.

Prior to recognition, the iris must first be located, isolated and segmented. The distinct shape as well as features of the iris, known as landmark features, are used to locate the iris in the image. If localization is not performed correctly this will lead to poor performance due to the inclusion of noise (e.g. eyelashes, sclera, pupil, eyelids, and specular reflection) in the image. Once the iris is isolated it is "unwrapped" from its natural circular form to a cartesian block of a fixed size. From the unwrapped iris a template is extracted by quantifying the textural data using 2D Gabor wavelet filters. Phasors, which include information on the orientation, spatial frequency and position of texture in the image, create a template from the original image. The template is known as the IrisCode ${ }^{\circledR}$, which can be visualized as a binary image (Figure 1.5). The IrisCode ${ }^{\circledR}$ consists exclusively of binary code which allows for easy matching through the 
use of the Hamming distance which lends itself to a test of statistical independence between the two IrisCodes ${ }^{\circledR}$. If the Hamming distance indicates that less than a certain number of bits are different, then the two templates fail the test of statistical significance and are deemed to be from different users [19].

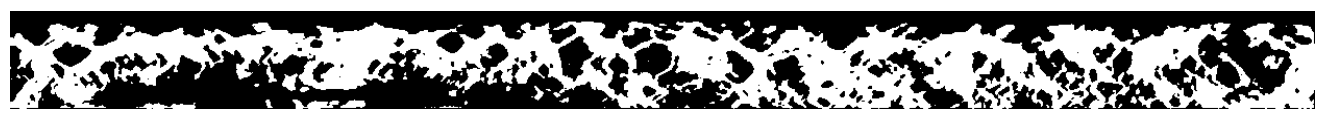

Figure 1.5: Graphical representation of a binary IrisCode ${ }^{\circledR}$. The hamming distance between two IrisCodes ${ }^{\circledR}$ is used to determine the matching score.

\subsection{Multibiometrics}

An identification system, whether biometric or not, that relies on only a single source of identification may not meet desired performance requirement. By incorporating multiple sources of information into an identification system, the limitations of a single identity management solution can be overcome. A popular trend is to integrate a biometric system with an additional identification technology [20] (smart cards, passwords, RFID, etc.) to form a dual-factor authentication system. Though this is an effective way to increase system performance, it now requires users to possess an item (smart card) or remember a password which are drawbacks that are not present in a biometric system. A multibiometric system, instead, increases the number of sources from which biometric information is collected. Multibiometric systems offer a multitude of benefits compared to traditional unimodal biometric system. Some of the benefits are described below [21]:

- Multibiometrics can potentially offer a significant increase in matching performance. The potential for improvement is dependent on the biometric modalities present as well as the fusion algorithm. 
- Multiple biometric systems may address the issue of non-universality and lack of population coverage. It is likely that if a person fails to enroll into a single biometric they will still have the ability to enroll into the second and, hence, still be capable of using the identification system.

- The addition of multiple biometric systems increases the difficulty of spoofing the system.

- By including multiple sources of information the system can more effectively address noisy data. The ability to negotiate noisy data is especially important when data is collected in adverse conditions.

- Multibiometric systems also increase the ability to continuously monitor or track individuals over a period of time. As an example, monitoring through audio/video may be implemented via face and voice recognition. If the system simply relied on face recognition, the ability to track would be lost once the face is occluded and voice recognition would fail if the individual fails to talk. By combining the two technologies it is more likely that the individual can be continuously identified either by a single trait or a combination of both traits.

- Multibiometrics increase the redundancy of the system allowing for the recognition system to continue to work even after the failure of one of the biometric sources.

Multbiometric systems can be comprised of a variety of different sources of biometric information. Multibiometric fusion can be accomplished through both the use of a single trait as well as multiple traits. Below is a list of possible scenarios in which fusion may be performed [21]:

- Multi-sensor systems: A single biometric trait is extracted multiple times using different extraction devices. Example: Collecting data of the same finger via the use of both a optical and capacitive fingerprint sensor.

- Multi-algorithm systems: A single biometric sample is processed by multiple algorithms. 
Example: A fingerprint image is processed twice, first with a texture-based algorithm and then again using a minutiae-based algorithm.

- Multi-instance systems: Multiple instances of the same biometric are used for recognition. Example: An iris system which captures both the user's right and left eye to identify the user.

- Multi-sample systems: Multiple samples of the same biometric are captured using a single device in order to account for variations of the biometric. Example: A face detection system captures multiple images of the same user to increase the probability that the subtle features of the face have been successfully captured.

- Multimodal systems: Combines multiple biometric modalities into a single biometric system. Example: A biometric system in which the user is required to interact with a fingerprint sensor as well as an iris camera.

\subsubsection{Multibiometric Fusion}

The additional biometric data which is collected in a multibiometric system must be fused from multiple sources to achieve a single result. Methods of biometric fusion can be classified into two categories: fusion prior to matching and fusion after matching [22]. Fusion prior to matching can be further classified into either sensor level or feature level fusion. Sensor level fusion is defined as the fusion of the raw data (e.g. images) from the capture device. Since multiple sources of the same biometric are required for sensor level fusion, it is only applicable to multi-sensor and multi-sample biometric systems. Feature level fusion is the combination of feature sets from multiple biometric sources. Feature sets can be both homogeneous (multiple measures from the same biometric source) or non-homogeneous(multiple measures from multiple biometric sources). The multiple biometric sources must be concatenated into a single feature vector, therefore not all feature sets are compatible. Features such as fingerprint minutiae and 
eigen-face coefficients can not be easily concatenated and, therefore, they are not candidates for feature level fusion.

Fusion techniques which integrate information after matching are separated into four categories: dynamic classifier selection, fusion at the decision level, fusion at the rank level and fusion at the match score level [21]. Dynamic classifier chooses the single biometric source which will provide the best result given a specific input pattern. Fusion at the decision level allows each biometric system to independently make a decision followed by a voting scheme. Voting schemes such as majority voting and weighted voting are among the most popular. This fusion scheme is popular among commercial biometric products since it only requires knowledge of the final matching decision which is typically easier to access compared to intermediate data. Fusion at the rank level is only available in systems where possible matches are ranked based on their likelihood of being the correct match. Fusion is based on the combined rank from multiple decisions. As an example, the final decision may be based on the mean of the ranks across multiple matches. Fusion at the match level is the combination of the match score from multiple sources. Match level fusion is commonly implemented by implementing an arithmetic or statistical operation on the multiple matching scores.

\subsection{Multispectral Iris Recognition}

\subsubsection{Multispectral Imaging}

Multispectral imaging is an imaging technique which was originally created for satellite imaging of geospatial structures. More recent applications include the processing of medical images [23] as well as face [24,25] and finger biometrics [26]. Multispectral imaging allows for the capture of information beyond the wavelength of visible light. This allows for additional information which is not visible to the human eye to be utilized. Hyperspectral imaging, a technology similar to multispectral imaging, collects large quantities of images simultaneously in adjacent bands across the electromagnetic spectrum. 
Iris imaging concentrates on both the visible (400nm-700nm) and near-IR (700nm-1000nm) bands of the electromagnetic spectrum. Due to biological variations of an individual's iris structure which has a distinctive effect on the iris color, imaging in varying ranges of the electromagnetic spectrum will elicit distinct textural information across the multiple channels. Due to biological diversity in individuals iris structure (color and texture), multiple channels may not perform equally well (quality of textural iris data in the image) across all users.

\subsubsection{Multispectral Iris Imaging}

A multispectral image is comprised of multiple images of a single object captured over various wavelengths of the electromagnetic spectrum. Typically, each image is comprised of a relatively small band (approximately 50nm) over a particular wavelength. Each channel of the image is represented in a two-dimensional matrix where each entry in the matrix represents the pixel intensity at a certain point in space. Multiple images can be concatenated in the third dimension so that multiple wavelengths can be viewed in a single color image (Figure 1.6).

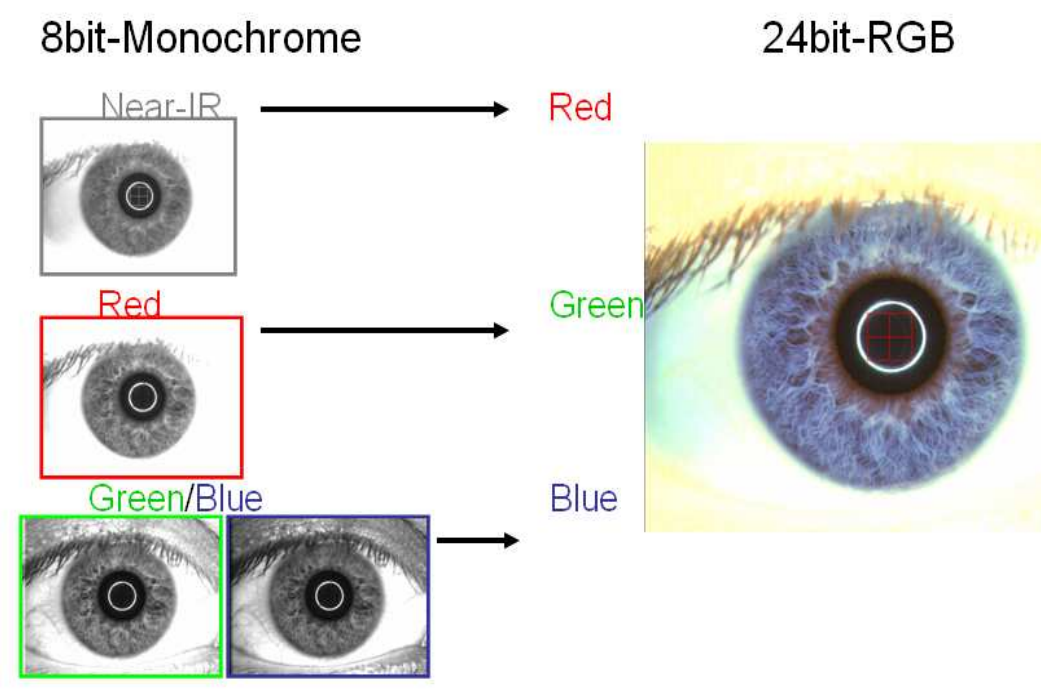

Figure 1.6: False color image and the four band channels which are extracted from it. 
To collect multispectral iris data the Redlake MS3100 multispectral camera was employed. The camera is composed of three charge coupled devices (CCD) and three band-pass prisms which reside behind a single lens. Once the light has entered the lens, four images are simultaneously captured across four different wavelength bands. Two Sony ICX205AL sensors whose spectral response ranges between $400 \mathrm{~nm}$ and $1000 \mathrm{~nm}$ with a peak of $550 \mathrm{~nm}$ capture both the IR and red components. Both the Green and Blue channels are captured on a single Sony RGBICX205 sensor. The sensor has a blue response ranging from $400 \mathrm{~nm}$ to $550 \mathrm{~nm}$ (peaking at $460 \mathrm{~nm}$ ) and a green response from $400 \mathrm{~nm}$ to $650 \mathrm{~nm}$ (peaking at $550 \mathrm{~nm}$ ) (Figure 1.7). The primary reason for using a single lens with multiple CCD sensors and prisms is that all four channels can be captured at the same time. Thus, there is no need for geometric registration since the images corresponding to multiple channels are captured simultaneously. Each channel is manually white-balanced prior to the image being created using a color checking card. Since both the green and blue channels are captured on the same CCD sensor their resolution is not as high as the IR and red channels. The sensor output from the IR and red image is 1300x1040 pixels with approximately 56,000 pixels comprising the actual iris. The blue and green channels are recorded at a resolution onefourth (260x348 pixels) of the IR and red channels. Each channel is then extracted and scaled to the size of the IR and red images using nearest neighbor interpolation. The acquisition device is designed with the following parameters: near-IR is captured with a center wavelength of $800 \mathrm{~nm}$ with a band pass of $60 \mathrm{~nm}$, red light is centered at $670 \mathrm{~nm}$ with a band pass of $40 \mathrm{~nm}$, green is centered at a wavelength of $540 \mathrm{~nm}$ and a band pass of $50 \mathrm{~nm}$, and the blue channel has a center wavelength of $475 \mathrm{~nm}$ with a band pass of $50 \mathrm{~nm}$.

An ophthalmologist's slit-lamp mount was used to provide a stable mount for the camera (Figure 1.8). To ensure that the iris is accurately imaged, the slit-lamp mount includes a chin rest to ensure the users head stays in proper position. Also the camera is placed on a mobile camera mount which can be finely adjusted in 3-dimensions to ensure the eye is accurately imaged. To provide illumination a broadband light source is employed in the form of a ring light that is placed between the imaging device and user. To ensure illumination across the whole 


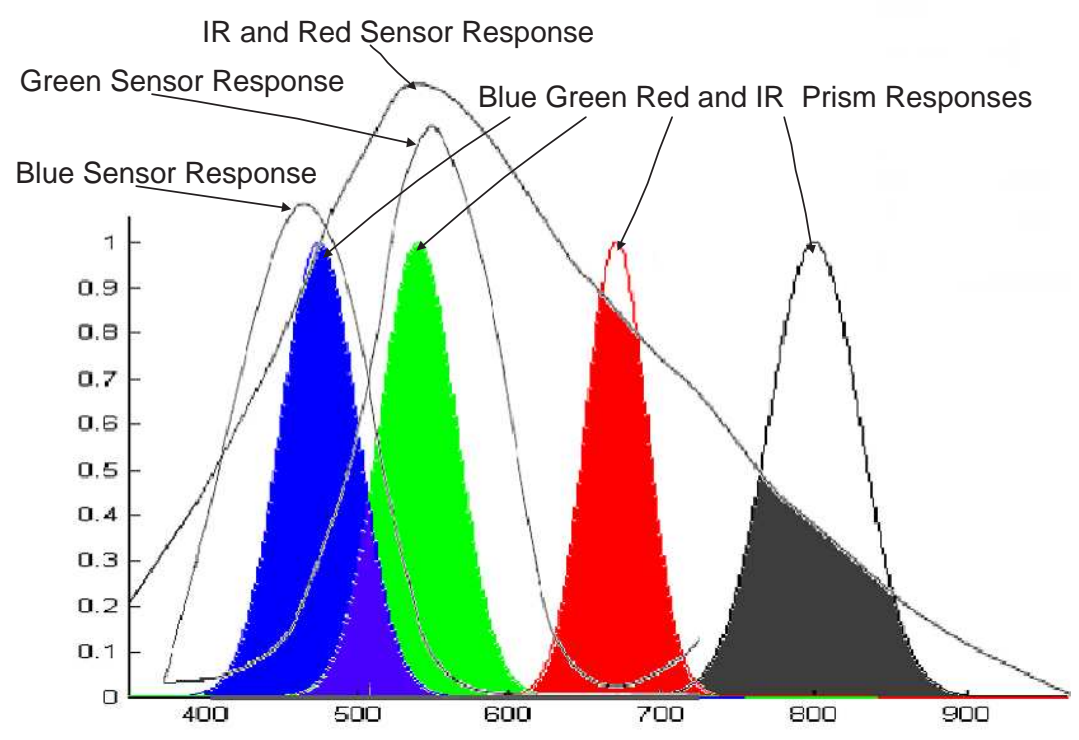

Figure 1.7: The normalized transmittance band-pass wavelengths for each prism and sensor of the multispectral device. Portions of the graph which are filled represent the combined response of the sensors and prisms.

spectrum the light source ranges from $350 \mathrm{~nm}$ to $1700 \mathrm{~nm}$. The light source is projected onto the eye through the use of a fiber optic light-guide which leads to a ring shaped light which provides a uniform circular illumination across the eye which does not obscure the view of the camera. Due to the reflective properties of the eye, a large ring of light is reflected off the cornea of the eye. This is a stark contrast to the small reflection points caused by LED based illumination which is common in most commercial iris image recognition systems. The only source of illumination is the ring light, all forms of ambient light are eliminated prior to image acquisition.

\subsubsection{Multispectral Image Data}

A database of 35 subjects, each consisting of 5 samples per eye was created using the multispectral device previously described. Since each of the user's two eyes are considered to be distinct they can be assumed to be two unique subjects, hence bringing the total number of users to 70. Each subject was classified based on color of the iris into three classes: Dark Brown, 


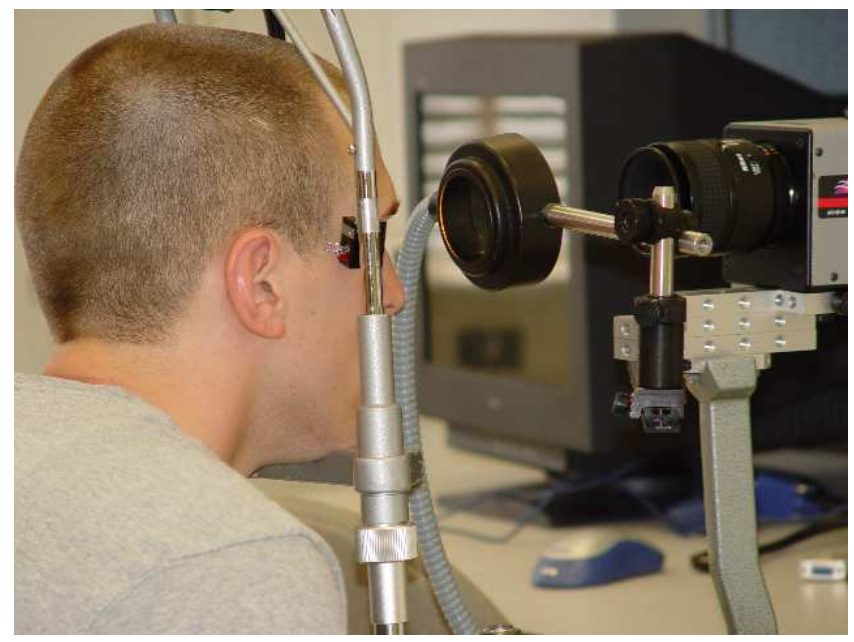

Figure 1.8: Demonstration of the multispectral camera capturing an image of a subject's eye.

Light Brown/Green, and Blue. The color of the eye was determined based on a visual examination of the image in the RGB color space. No attempts to automatically determine the iris color were made due to the difficulties arising from the rapid variations in texture chromaticity created by images of such high resolution. The images are typically viewed as either CIR (color infrared) or RGB images. A CIR image is created by mapping the IR component of the image to the first channel, then mapping the red and green components to the second and third channels, respectively. The three channels are then concatenated in a similar manner as the RGB image (Figure 1.9). The blue channel is discarded.

\subsubsection{Color (RGB) Iris Database}

A second, more robust iris database was used for analysis of color. The images are 24-bit three channel RGB (Red Green Blue) color images 1.10. The University of Beira Interior iris database (UBIRIS) is composed of 1877 images from 241 users $^{2}$. Many of the images within the

\footnotetext{
${ }^{2}$ http://iris.di.ubi.pt/
} 

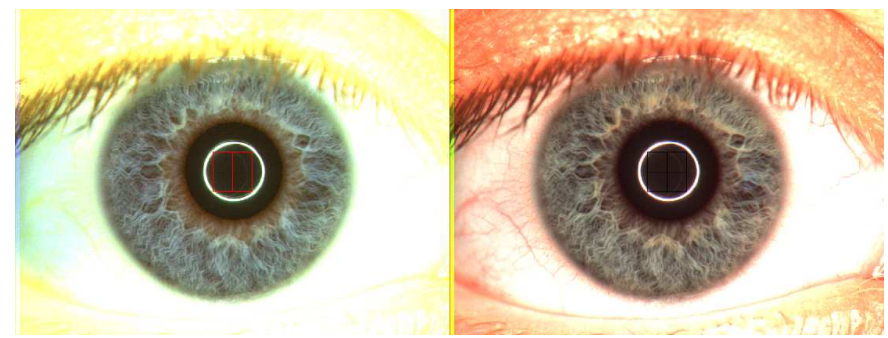

Figure 1.9: Visual comparison of the color RGB image (left) and the CIR image (right).

database were not taken under ideal conditions, leading to varying amounts of occlusion from the eyelids and eye lashes. For this research 127 users were selected from the database at random. Five images per user were taken creating a database of 635 total images. The database comprised of 63 users with brown eyes, 40 users with light brown/green eyes and 23 users with blue eyes.

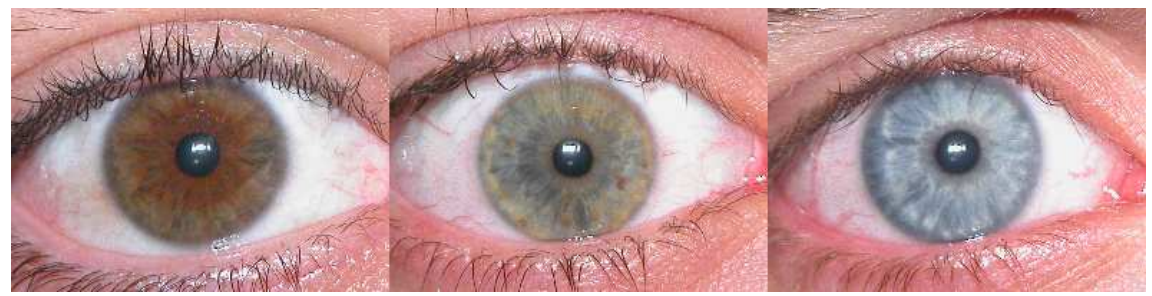

Figure 1.10: Typical examples of color images found within the UBIRIS database.

\subsection{Color Space Analysis}

With the inclusion of color in iris recognition, the representation of color must be considered. When analyzing color images for this work, the representation of chromaticity and luminance were both carefully considered. The representation of luminance, the measurement of the amount 
of light reflected at a given angle, is crucial for iris recognition [27]. Since the texture of the iris is represented most vividly via the luminance of the image, it is important that the luminance be well represented. Chromaticity, which is introduced with the notion of color, is the quality of color as determined by the hue and purity [28]. The impact of chromaticity on texture-based iris recognition is well documented throughout this work.

\subsubsection{Color Model}

To mathematically describe colors, a color model is used. A color model is an abstract mathematical model which, through the use of tuples, quantitatively represents the visualization of color [29]. Typically three (or four) values (color components) are used to represent the model.

The RGB generic color model is the most convenient and popular model for processing digital images (Figure 1.11). RGB is an additive color model which combines the three primary colors in predefined proportions to produce the full gamut of colors. The term additive color refers to the fact that colors are added together to create new colors from the initial state of black (absence of all color). The standard RGB (sRGB) color space is commonly used for computer based applications and will be the RGB color space of choice throughout this work.

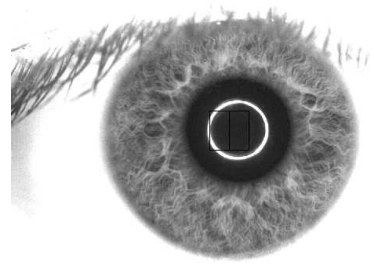

(a) Red Band

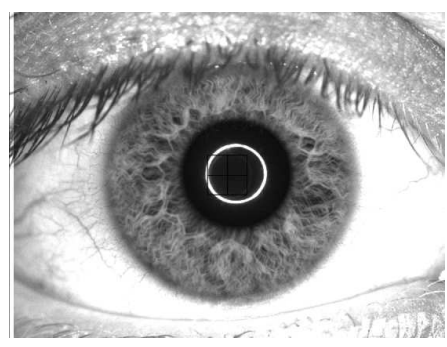

(b) Green Band

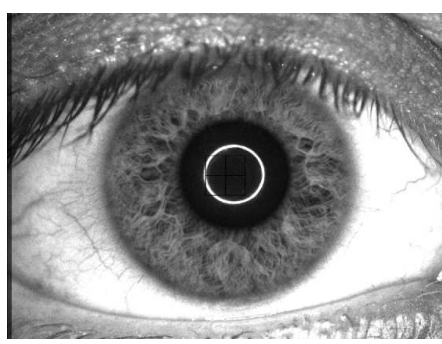

(c) Blue Band

Figure 1.11: Individual components of a sRGB image.

The International Commission on Illumination (CIE) Lab color space can be decomposed into three channels: Luminance (L) and the color components a and $\mathbf{b}$ (Figure 1.12). CIE Lab 
is derived from the CIE XYZ color space therefore it is impossible to go directly from a common RGB color space to the CIE Lab space. However, any RGB color space can be easily converted to CIE XYZ color space then subsequently converted to CIE Lab. CIE Lab is known as a color-opponent space since it mimics the way the human visual system interprets color. Cones and rods, which are responsible for interpreting color in the human eye, respond to overlapping wavelengths of light [30]. Due to this, it is more efficient for the visual system to interpret the differences between the responses of multiple cones instead of the exact response of all cones. A color-opponent space mimics this response by recording the difference in opponent channels. The black versus white variation, or light-dark variation is known as luminance [31]. The color channels $\mathbf{a}$ and $\mathbf{b}$ show the variation in red versus green and blue versus yellow, respectively. Since CIE Lab closely mimics the eye's ability to determine color, this color space allows for more perceptual uniformity compared to other color spaces. This allows for more accurate color balance corrections and adjustments of contrast. Such modifications in other color spaces are not as accurate since one cannot adjust a characteristic of an image (lightness or color) without affecting the other.

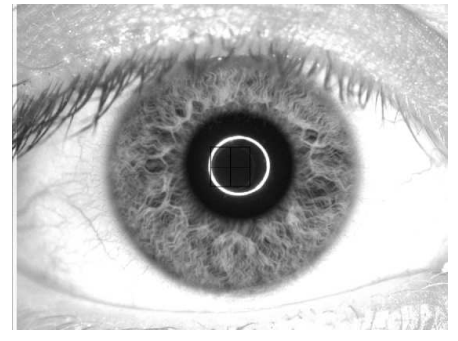

(a) Luminance

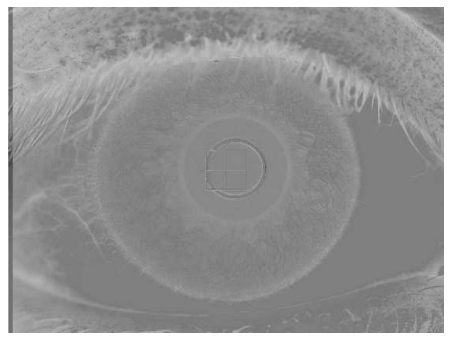

(b) Red versus green component

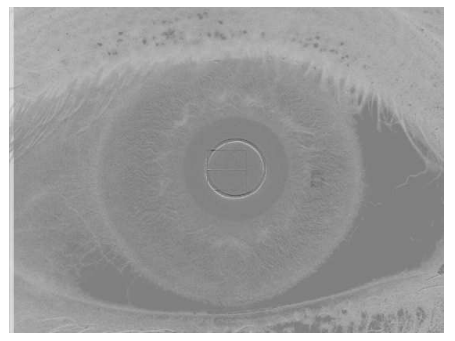

(c) Blue versus yellow component

Figure 1.12: Individual components of a CIE Lab image.

$\mathrm{YCbCr}$ is a discrete three component color space used commonly in videos (Figure 1.13). Similar to CIE Lab, YCbCr is an opponent-color system. The first channel, Y, is the luma of the image. Luma is the brightness of the image and unlike the similar term luminance, which is 
formed based on the weighted sums of the linear RGB components, luma is the weighted sum of the gamma-compressed corrected RGB image [32]. The chrominance (chroma) of the image is held on the other two components. The blue chroma component is labeled $\mathbf{C b}$, and $\mathbf{C r}$ is the red chroma component. $\mathrm{YCbCr}$ is not an absolute color space, but instead a way of encoding the RGB color model. Therefore, the actual color representation is dependent on the RGB color space in which it was derived from. Though the color representation is similar to RGB, the advantages of it being a color-opponent space justifies our use of this non-absolute space.

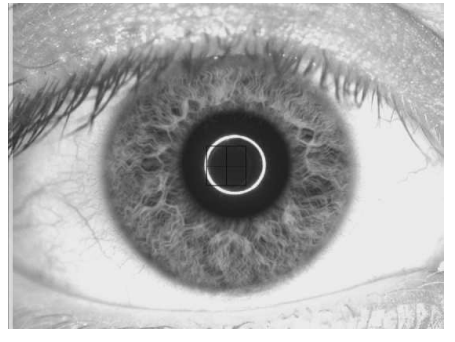

(a) Luma

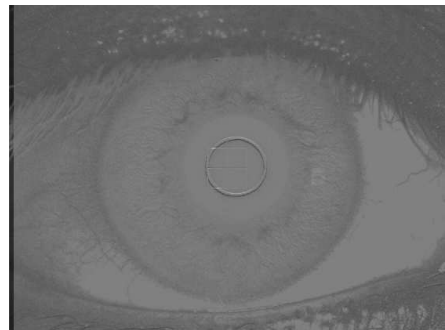

(b) Chroma Blue

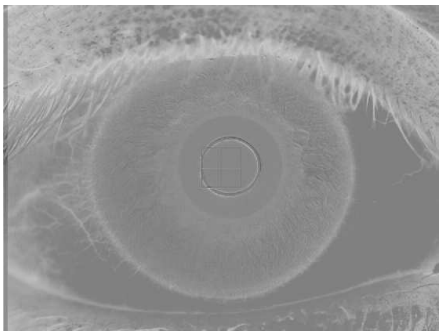

(c) Chroma Red

Figure 1.13: Individual components of a $\mathrm{YCbCr}$ image.

The Hue Saturation Value (HSV) color model, also known as Hue Saturation Brightness (HSB), is a popular color model for computer graphics applications (Figure 1.14). The hue of an image refers to the pure color. A pure color is a color derived from the three primary colors, but without the presence of white or black to lighten or darken the color. For most applications Hue ranges from $0-360$, where each value corresponds to one color (Figure 1.15). Saturation is the intensity of the color, typically ranging on a scale from $0-1$ where 0 shows no color (which appears as a shade of grey) and 1 represents a deep, intense color. The saturation of color is dependent on the combination of light intensity and it's distribution across the spectrum of multiple wavelengths. Value is the measurement of brightness of color, ranging from $0-1$. A value of 0 represents black and a value of 1 represents a value of white or a bright, saturated color. Unlike other color models, HSV is not device independent. HSV can only be defined relative to 
its respective RGB intensities. HSV is also a popular color model due to its similarities with the way humans perceive color. Unlike additive and subtractive color models which define color based on the combination of primary colors, HSV defines color in terms more similar to the way the human brain interprets it.

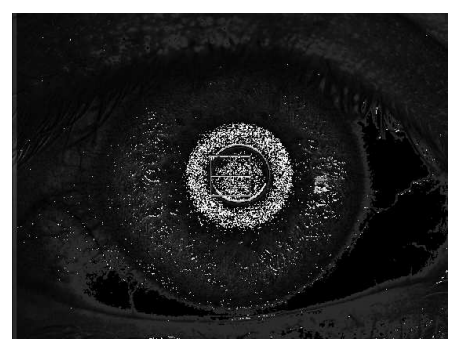

(a) Hue

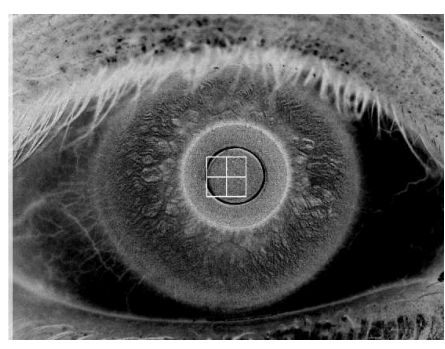

(b) Saturation

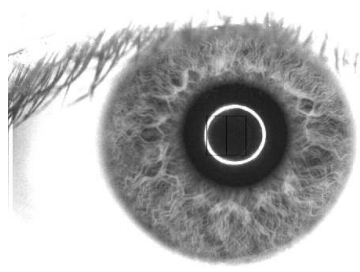

(c) Value

Figure 1.14: Individual components of a HSV image.

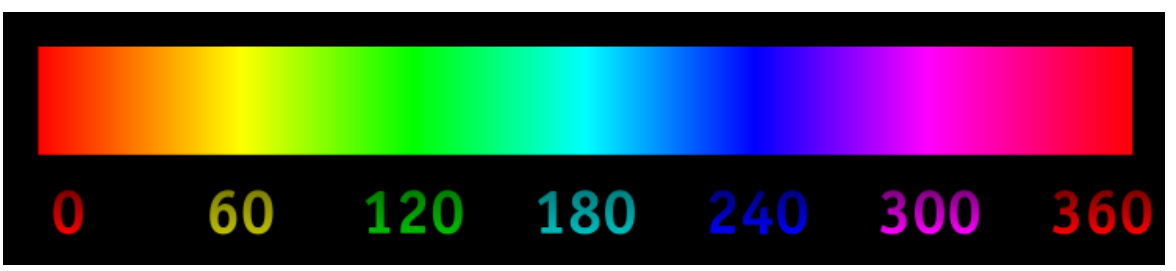

Figure 1.15: Visual representation of the hue scale showing the corresponding color value.

Cyan, Magenta, Yellow and Key (Black) (CMYK) is a subtractive color model (Figure 1.16). Opposed to an additive color model, a subtractive model begin with no color (white) and, through the addition of various portions of each color channel, creates all colors of the gamut. If the three primary channels cyan, magenta, and yellow are fully saturated at a single point, the result is the color black. Since the CMYK color model is the primary color module used for color printing, a 
fourth channel of black was introduced, which allows printers to use the less expensive black ink instead of a combination of the three more expensive colors.

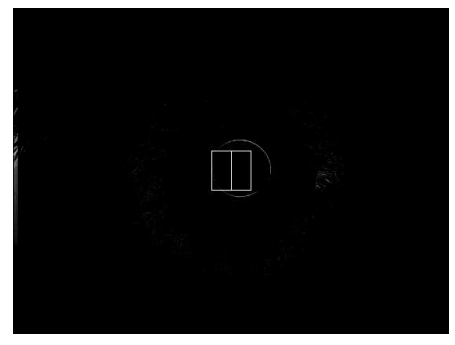

(a) Cyan Component

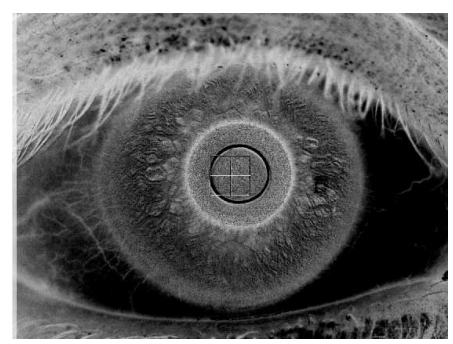

(c) Yellow Component

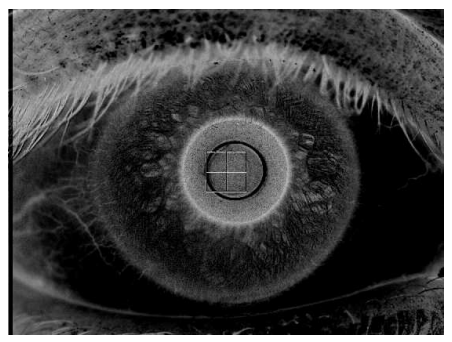

(b) Magenta Component

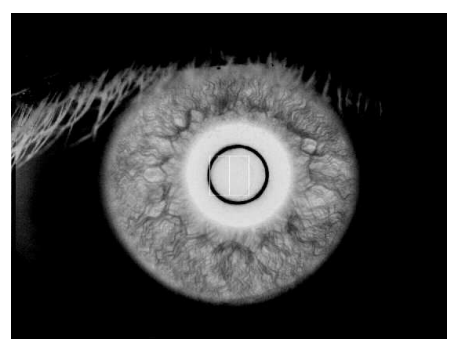

(d) Black Component

Figure 1.16: Individual components of a CMYK image.

\subsubsection{Color Space}

The precise distribution of how the multiple channels of the color model are interpreted is known as a gamut. Furthermore, the gamut is the portion of the color space which can be represented or reproduced. The gamut range is typically specified using the hue-saturation plane, since many color systems can produce colors within a wide intensity range of their respective color gamut. Though it is not possible for any single gamut to display the entire visible color space model, color gamuts provide good approximations which pass the limitations of human perception [33]. The most common visual representation of a gamut is through the CIE 1931 
chromaticity diagram (Figure $1.17^{3}$ ). The triangular shape of the gamut is due to the fact that most color models are represented with three primaries. A full gamut must be represented in 3D space since the accessible gamut also depends on brightness (Figure 1.184). The combination of the color model and gamut defines the color space.

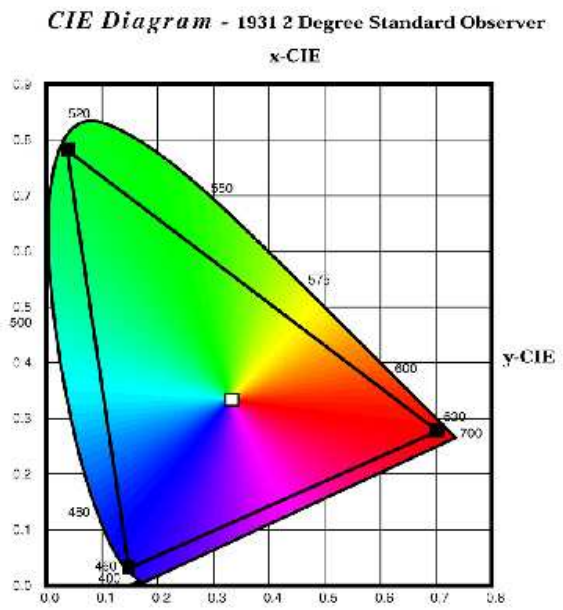

Figure 1.17: The gamut of all representable colors in the sRGB color space.

\subsection{Literature Review}

\subsubsection{Multispectral Iris Recognition Analysis}

Previous work in multispectral iris recognition has included segmentation, recognition and liveness detection. Using four narrow band of the electromagnetic spectrum to capture a four channel(near-IR, red, green, blue) multispectral image Boyce et al [18] developed a novel iris

\footnotetext{
${ }^{3}$ www.microsoft.com

${ }^{4}$ www.brucelindbloom.com
} 


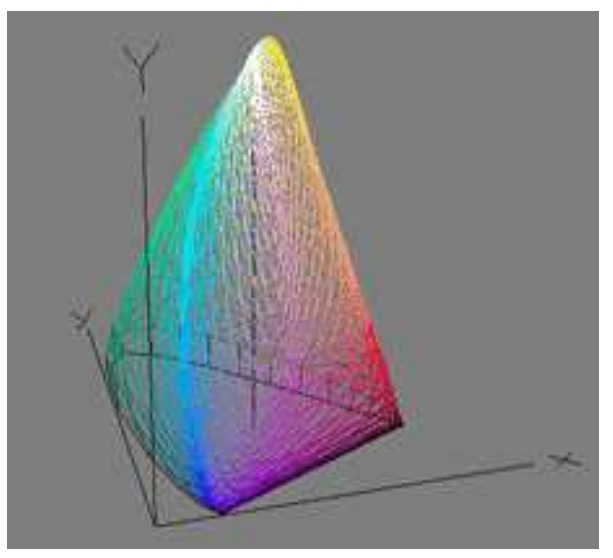

Figure 1.18: 3D gamut of the CIR XYZ color space. The third dimension allows for the inclusion of brightness into the gamut.

segmentation and recognition schemes. By eliciting information across all channels of the multispectral image, instead of just near-IR as in many commercial iris recognition systems, a more accurate iris segmentation was achieved. Segmentation is based on Bayes classifiers to isolate the non iris content of the image from the iris content. Segmentation takes place in two steps, pupil segmentation and iris segmentation. An encoding scheme utilizing the reflectance across each channel of the iris image is described. All four channels are utilized by creating feature vectors of the Euclidean distance between the two neighboring channels. The feature vectors are then used for matching. A software based spoof detection algorithm utilizing multispectral imaging is also introduced. Picture, video, printed contacts and prosthetic eyes are all used to try and spoof the system. Spoof irides are detected by measuring the radius of the pupil. A real pupil will have subtle changes in it's radius due to constriction and dilation overtime. By tracking the pupil over a short period of time it can be determined if the image is of an actual iris. To prevent video spoofing the power spectrum is analyzed to determine if periodic peaks appear. Periodic peaks in the decomposed power spectrum signal indicates the presence of pixels thus verifying the use of a monitor. 


\subsubsection{Fake Iris Detection through Variations of Reflectance Ratios}

Further work into spoof detection using multispectral imaging was done by Park et al [34]. A fake iris is detected based on the changes in reflectance ratios between the sclera and pupil regions of the eye. Since the sclera and pupil of an actual eye are two separate structures and composed of different tissue, they will have different reflectance ratios if imaged at various wavelengths of the electromagnetic spectrum. The dense, fibrous and collagenous structure of the sclera decreases in reflectance as the wavelength of the illumination increases [35]. While the reflectance from the melanin of the iris increases with the same increase in illumination wavelength [36] causing the reflectance ratios to increase as the spectral wavelengths increase. Fake irides which are printed are composed of a single material in both the iris and sclera region; therefore as the illumination wavelengths increase, the reflection ratios between the two will not. Other spoofing techniques such as fake contacts and prosthetic eyes which are comprised of two different tissues in the iris and sclera region have a reflectance ratio which is outside the expected ratio of a real iris and can be detected.

\subsection{Contributions of this Thesis}

The objective of this thesis is to consider salient issues in multispectral iris recognition, fusion, and color space analysis. These contributions are listed below:

- A novel score level fusion algorithm which combines the evidence of each channel of the multispectral image to generate a single matching score for each comparison. The fusion algorithm is based on the textural characteristics exhibited in each channel of the iris image.

- Analysis of color space transformations and their effect on matching performance. The matching performance is analyzed across the RGB, CIE Lab, HSV, CMYK and YCbCr color spaces to determine if an optimal color space exists for color and multispectral iris recognition. 
- Analysis of color image enhancement in the CIE Lab, HSV, and YCbCr color spaces as well as its effect on matching performance is studied in this work.

The organization of this work is as follows. Chapter 2 explores score level fusion of multispectral iris images. The chapter, first details the preprocessing, segmentation and encoding schemes. Both Gabor and local binary pattern (LBP) based encoding approaches are discussed. The proposed score level fusion algorithm is compared to other fusion techniques that are based on the characteristics unique to eyes of different color. The third chapter focuses on color space analysis and it's effect on evaluating the performance due to color images. Work within this chapter focuses on the high correlation eye color has on matching performance across specific bands of the visible spectrum. The final chapter of this work reiterates the contributions of this thesis as well as suggests possible future work in the area of multispectral iris recognition. 


\section{Chapter 2}

\section{Dynamic Score Level Fusion}

\subsection{Motivation}

Contrary to most commercial iris recognition systems which only image within a narrow nearIR band, multispectral sensory allows for imaging across the spectrum. The result of imaging in multiple spectral channels is the availability of additional information through multiple images of the iris each captured at a unique channel. To justify the capturing of additional images, which may result in additional sensor/processing costs for capturing devices and additional storage and illumination requirements, a benefit in matching performance over traditional iris recognition systems must be demonstrated. This work shows that through a dynamic score level fusion algorithm improved matching performance can be seen. By analyzing all four channels (IR, red, green, blue) of the multispectral image and dynamically emphasizing the channels which show a propensity for high performance, a dynamic score level fusion algorithm can be devised. Isolating a unique set of color channels for each image creates a mechanism for score level fusion which shows a greater performance than each channel's independent performance. 


\section{$2.2 \quad$ Iris Preprocessing}

Since the objective of this work is score level fusion, the iris preprocessing, specular reflection, and segmentation algorithms developed by Boyce et al. were used [18]. Prior to encoding, preprocessing is applied to the images to ensure optimal quality and performance. To enhance the structural components across the different spectral channels of the image, adaptive histogram equalization is performed in the CIE Lab color space. All intensity values of the image are contained in the $\mathbf{L}$ parameter in the Lab color space, while the colors between green and magenta are represented by the a parameter, and colors between blue and yellow by b. By performing adaptive histogram equalization on the $\mathbf{L}$ parameter exclusively, we are ensured that the original colors of the image are not modified. Therefore, the intensity values can be moderated without affecting the color components, allowing for greater contrast and improved textural quality (Figure 2.1).
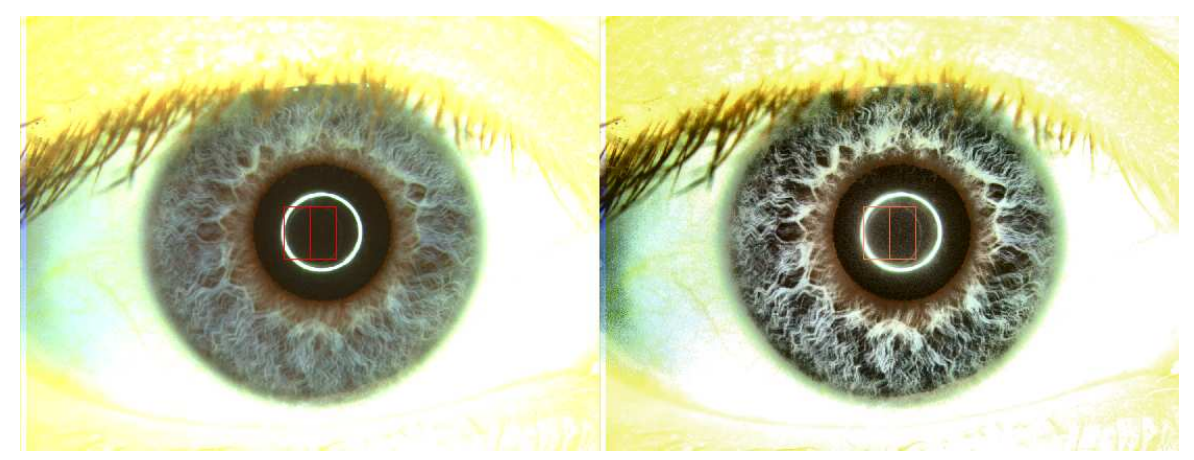

Figure 2.1: An iris image before (left) and after (right) textural enhancement using Lab histogram equalization.

Examining the initial images will show that a reflection of the illumination source can be seen across all channels. This reflection, known as specular reflection, can be seen as an ellipse approximately at the center of the iris. Specular reflection is a result of the source light (ring light) reflecting off the moist, highly reflective surface of the cornea. During image collection, 
precautions are taken in an attempt to limit the specular reflection to the pupil only, which would not have an effect on the textural content of the iris. Though this did help in reducing the reflection within the iris, it was not possible to completely limit the reflection to the pupil in all cases. Reflection due to ambient light played no role in the imaging process since all other light sources besides the source light were removed prior to imaging. Due to a variety of factors, such as unintentional movement of the eye (saccade) and head, it is not possible to completely restrict the specular reflection to the pupil; therefore, some removal of specular reflection from the iris will be required.

Prior to the removal of specular reflection, the area affected by specular reflection must first be detected. Since the result of specular reflection in these iris images is a high intensity white light, the specular reflection appears as the maximum intensity value within the image (in an 8-bit image this value is 255). Therefore, the reflection can be easily detected in each channel by thresholding using a high, predefined intensity. To ensure optimal detection, the high intensity thresholding is applied across all four channels of the multispectral image. The resulting binary images are then fused using a logical AND operation. to create a pupil mask. The pupil mask image is composed of pixels that have a high intensity value across all four binary images. This ensures that spurious pixels due to noise or other factors are removed leaving only a mask of the specular reflection. To ensure connectivity through the whole ring (the resulting shape of specular reflection) the morphological operator "close" is used to replace unseparated regions. Next, the center coordinates and radius are approximated by fitting a circle to the mask. Figure 2.2 gives a visual representation of the specular reflection isolation algorithm. A coding feature is now introduced which optimizes the efficiency of the inpainting algorithm. A rectangular region of interest (ROI) which spans just outside the mask is extracted. This allows us to sample the portion of the image which contains the specular reflection exclusively. The mask now contains the portions of the image which will undergo texture synthesis as described below.

The adverse effects of specular reflection within the iris are remedied through Markov Random Fields. Let $I$ be the initial iris image, the pixel $p \in I$ has an unknown value. In order to determine or 


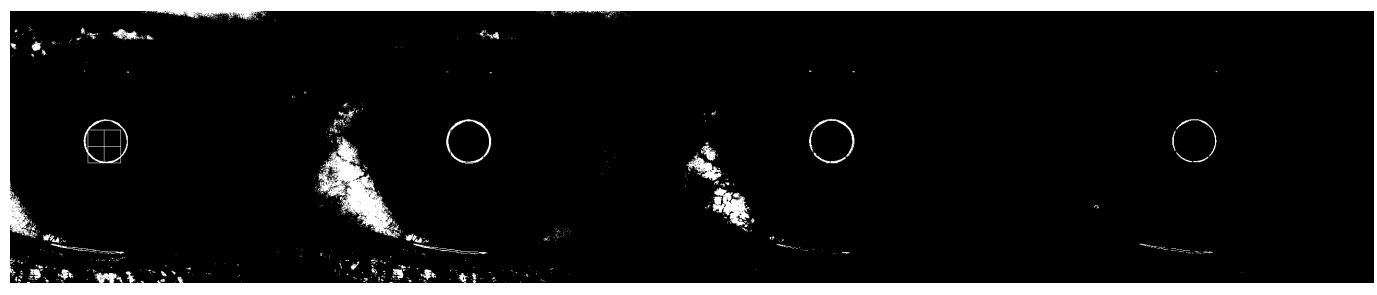

(a) Ring light across all four channels (from left to right: IR, red, green and blue).

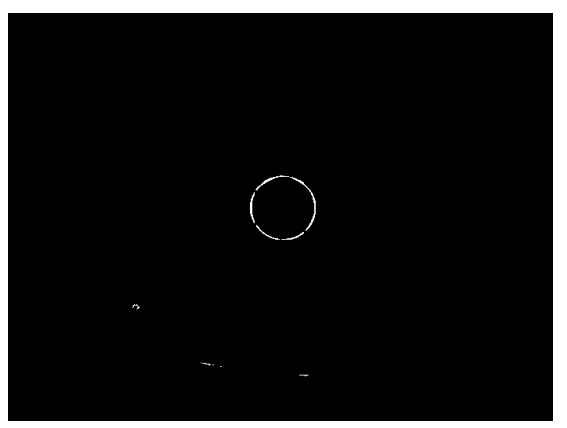

(b) Result of logical AND operation.

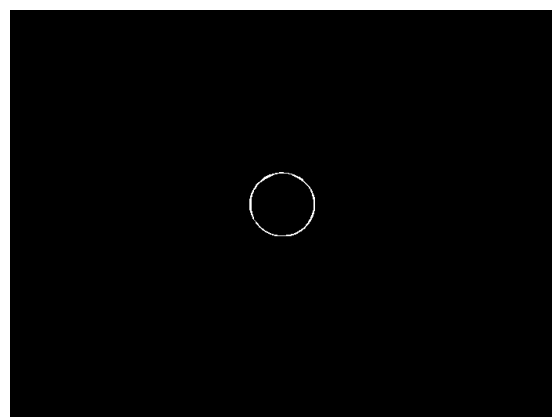

(c) Result of morphological closing and largest object selected.

Figure 2.2: Steps to isolate the specular reflection caused by the fiber optic ring light. 
synthesize the value of $p$, an approximation of the conditional probability distribution $P(p \mid \omega(p))$ must be known and sampled. Here the function $\omega(p)$ is a square mask of a fixed size centered at pixel $p$. A variation of the nearest neighbor technique as described below is implemented

$$
\omega_{o p t}=\operatorname{argmin}_{\omega}(d \omega(p), \omega) \subset I_{\text {fsamp }} .
$$

where $w_{\text {opt }}$ is the closest match to the true pixel value, $I_{f s a m p}$ is a finite sample space of the initial image and $d(\omega(p), \omega)$ is the distance between the two image patches $\omega(p)$ and $\omega$. The distance metric, $d$, is measured via the normalized sum of squared difference metric convolved with the two dimensional Gaussian kernel $d$.

$$
d=d_{S S D} * G
$$

where $d_{S S D}$ is the sum-of-squared difference metric and $G$ represents the Gaussian kernel. The ROI is inpainted on a pixel-by-pixel basis to ensure that optimal texture quality is achieved. Results of the inpainting process can be witnessed in Figure 2.3.

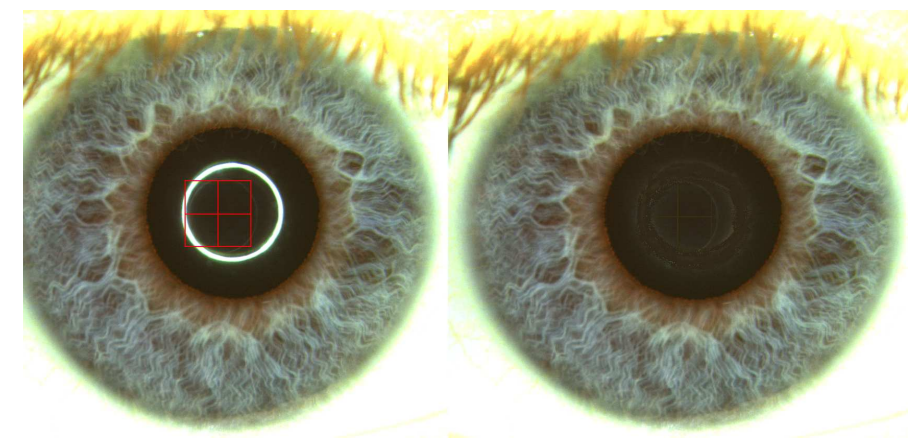

Figure 2.3: The original (left) image which includes specular reflection compared to the final impainting results (right). 


\subsection{Segmentation}

Iris segmentation consists of multiple operations: the localization of the iris, normalization and unwrapping of the iris and creation of a mask to remove non-iris components from the iris template. Irides are localized within the image using a multispectral iris segmentation algorithm [37] designed specifically to work across multiple color channels. The segmentation algorithm is divided into two stages: pupillary boundary detection (region between the pupil and iris) and limbic boundary detection (region between the iris and sclera). By eliciting the information across all channels of the multispectral image, a more robust segmentation is achieved utilizing a Bayesian classifier. To deduce the pupil masks using the Bayesian classification, first, the region which corresponds to the pupil must be determined. A dynamic thresholding technique is applied to establish the pupil mask in a single channel. Typically, the Infrared channel is used to generate the pupil mask. Since the pupil is usually the darkest part of the eye, a simple binary thresholding can be applied to elicit a large portion of the pupil (Figure 2.4). Initially, the threshold is set to zero plus the dynamic threshold $\left(D_{t h}\right)$. All pixels less than the threshold are set to 0 while all other pixels are set to 1 :

$$
I(t)= \begin{cases}1, & \min +D_{t h}(t-1)>I(t-1), \\ 0, & \min +D_{t h} \leq I(t-1) .\end{cases}
$$

where $I$ is the binary image after thresholding. The process is repeated until the sum of all pupil pixels $(I=0)$ is greater than the expected size of the pupil. With each iteration the threshold $\left(D_{t}\right)$ is incremented by 5 until the threshold for pupil size is achieved. After thresholding, spurious features may still exist in the image. Morphological operators such as close or hole fill may be performed to eliminate any noise from the mask. In most cases the eyelashes are very similar to the pupil in pixel intensity. Therefore, during thresholding, it is common for some of the eyelashes to be included in the final mask. To alleviate this, a check of connectivity is used to detect and eliminate eyelashes from the mask. Since the pupil is much larger than an eyelash in 
the $y$ direction, each column of the mask is examined to determine maximum connectivity.

$$
C_{y}=\sum_{r=1}^{N} P_{\text {con }}(r, c),
$$

where $c$ and $r$ are the rows and columns, respectively, $N$ is the total number of rows in the mask and $P_{c o n}$ is the approximate diameter of the object in which the pixels $c$ and $r$ are confined. Only the largest object is retained(Figure 2.5).

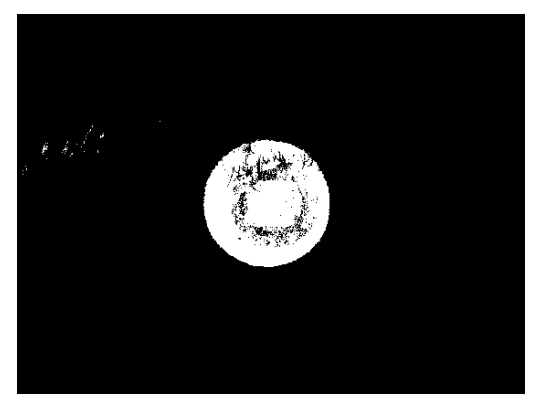

Figure 2.4: Binary iris image after pupil thresholding.
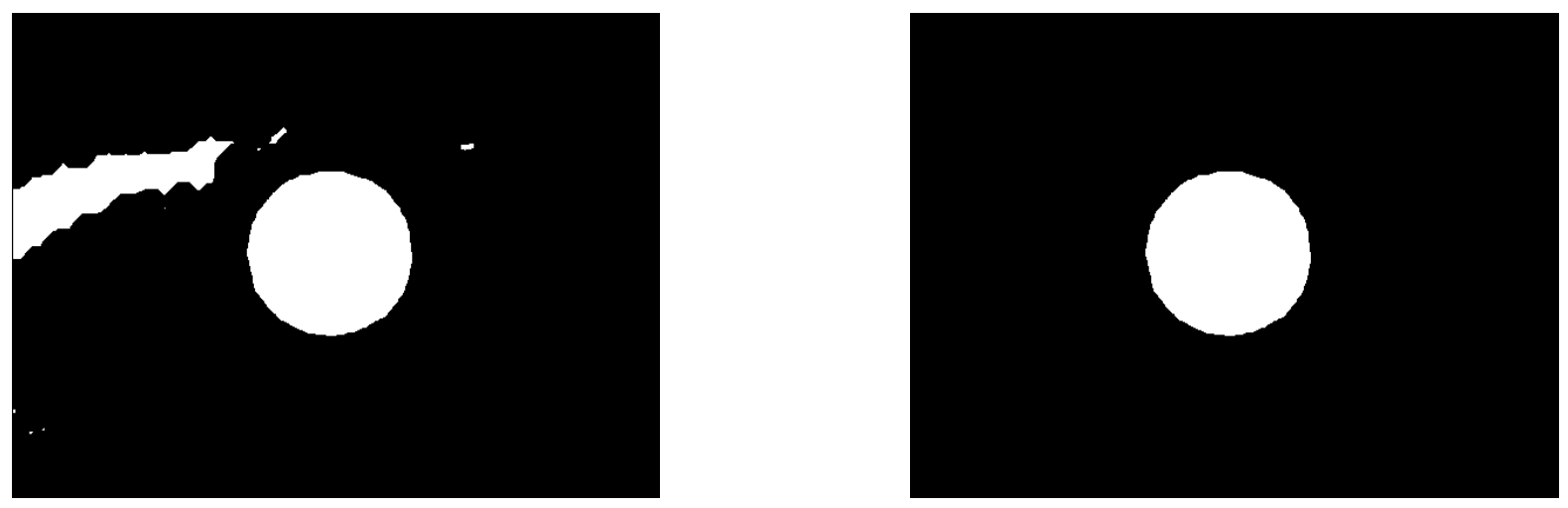

(a) Segmented image after morphological closing. (b) Pupil mask after detection of largest object.

Figure 2.5: Segmented Pupil 
To segment the limbic boundary, first the iris is first localized in the IR channel. This rough estimation of the extent of the iris removes pixels which are not likely to correspond to the iris. To achieve localization, two regions of interest (ROI) are selected at either side of the segmented pupil and extended radially into the sclera. Next the ROI are decomposed into 1D signals by calculating the radial mean across the columns. The partial derivative of each 1D signal is found using the finite differences across the means of each block.

$$
X_{f d}=X_{m n s}\left(x_{0}+\Delta x\right)-X_{m n s}\left(x_{0}\right) .
$$

Here $x_{0}$ is the current position along the $1 \mathrm{D}$ mean vectors $\left(X_{m n s}\right), \Delta x$ is the sampling position and $X_{f d}$ represents the finite difference. The maximum of the finite difference gives an approximation of the radius on each side of the pupil. This provides an estimate of the greatest changes in intensity across the image as well as assists in the approximation of the iris's radius. Since the limbic boundary is contained in the positive derivatives of the 1D signal the negative portions can be removed. The signal is then squared to emphasize peaks, and the maximum value, which represents the limbic boundary is detected. To restore the column from the downsampled signal the maximum value of the final signal should be multiplied by the rate at which it was originally downsampled. Since two regions of interest were extracted from the initial image there will be two radii, the largest of the two should be chosen for the initial limbic mask.

Samples representing each pattern class must be defined in order to obtain the mean vectors and covariance matrix. All channels of the multispectral image (IR, R, G, B) must be registered spatially and concatenated in the third dimension. Thus, every pixel is a combination of the four channels and can be simultaneously extracted from the iris and non-iris pattern masks. Next, the mean vectors and covariance matrices are computed from the newly created masks. The Bayes classification is computed using the covariance and mean vectors of the image. Since it is difficult to preclassify the components of the iris, only two masks are typically selected, one of the iris and one of the non-iris (Figure 2.6). Thus a binary image is created, which also contains structural components of the iris (such as crypts). To improve segmentation performance the following procedures must be implemented. To determine a more precise iris radius the localiza- 
tion approximation is again applied in the same manner as described above. After calculating a second radius, we can reclassify iris pixels which were misclassified as non-iris (such as crypts) if it falls within the calculated radius. Now the Bayesian classifier can be used again, but this time with three target classes: iris pattern (previously classified), non-iris pattern which was previously classified as iris pattern and non-iris pattern which was properly classified as non-iris pattern. Though the second Bayes classifier aids in the detection of the iris, it does not detect the true limbic boundary. A combination of both classifiers will provide better results. The portion of the image above the pupil is a binary image of the second class. The bottom half of the image is comprised of the binary image of the first class. Again, by applying the ROI boundary, all of the non-iris pixels outside the iris will be removed. Noise within the iris is removed with morphological operators and filtering operations. Once the iris has been localized, the pixels pertaining to the iris must be isolated, unwrapped and normalized. Our approach is based on Daugman's "Rubber Sheet Model" (Figure 2.7) where each point in the Cartesian coordinate system is mapped to its polar coordinate equivalent and then unwrapped to form a rectangular approximation of the original iris image [38].

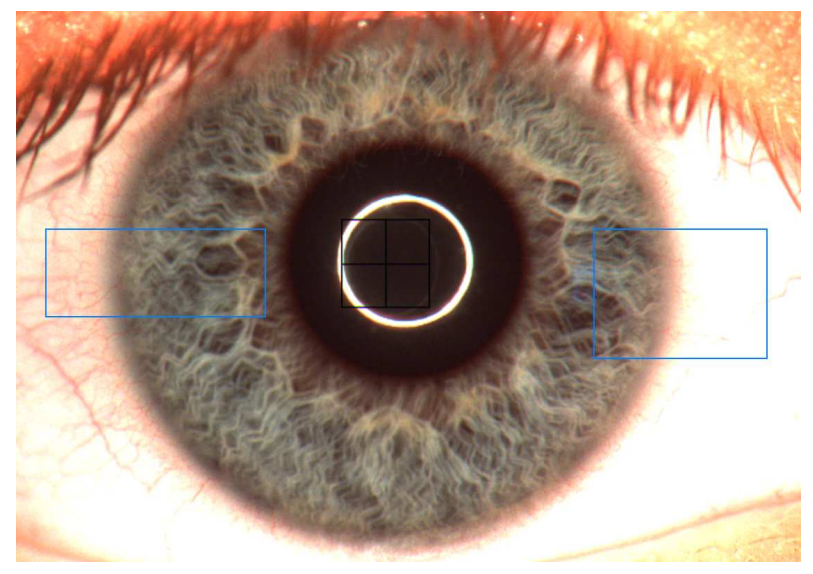

Figure 2.6: Localization of the regions of ROI within in the iris. 


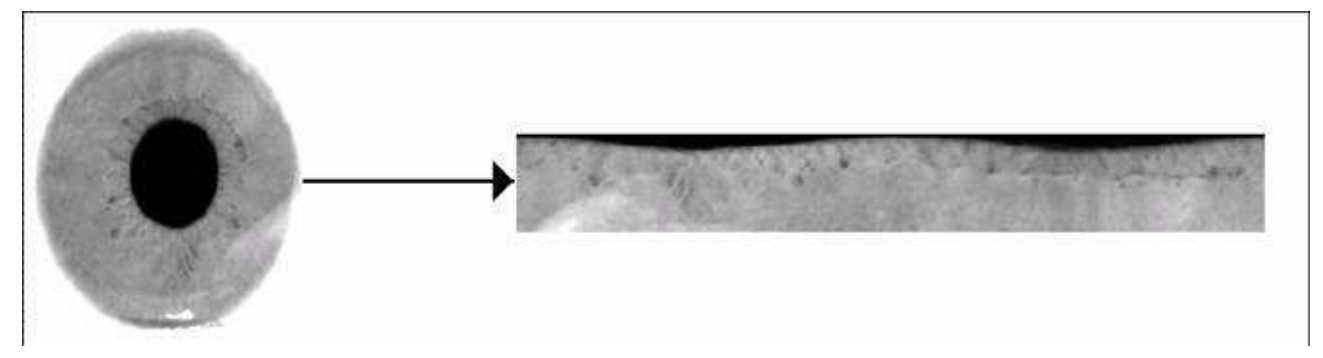

Figure 2.7: Once the image is segmented it is unwrapped and normalized into a fixed sized template.

\subsection{Encoding and Test for Statistical Independence}

A classical texture-based encoding and matching scheme has been implemented to test the efficiency of the score level fusion algorithm. Again, like segmentation, developing a novel encoding scheme is not within the scope of this work and therefore, the texture-based encoding algorithm described below makes an excellent candidate.

Once the iris has been located and isolated, the textural information must be quantified in a manner that will allow for two irides to be compared by a test of statistical independence. This process is initiated via a two dimensional wavelet demodulation. Demodulation extracts the phase information from the iris image using 2D Gabor wavelets [39,40]. The 2D Gabor filter is defined in the cartesian image plane $(\mathrm{x}, \mathrm{y})$ as

$$
G(x, y)=\exp \left\{-\pi\left[\frac{\left(x-x_{0}\right)^{2}}{\alpha^{2}}+\frac{\left(y-y_{0}\right)^{2}}{\beta^{2}}\right]\right\} \exp \left\{-2 \pi i\left[u_{0}\left(x-x_{0}\right)+v_{0}\left(y-y_{0}\right)\right]\right\} .
$$

where $\left(x_{0}, y_{0}\right)$ specify the center of the Gaussian portion of the filter, $\alpha$ is the width of the filter, $\beta$ is the height of the filter, and $\left(u_{0}, v_{0}\right)$ specify the modulation with frequency $\omega_{0}=\sqrt{u_{0}^{2}+v_{0}^{2}}$ and orientation $\theta=\arctan \left(\frac{v_{0}}{u_{0}}\right)$ [41]. Due to dilation, constriction, as well as the structural anatomy of the iris, as you move radially towards the limbic boundary of the iris the textural features change dramatically. To account for this, three Gabor filters, all with the same orientation 
$\left(0^{\circ}\right)$, but varying scales and frequencies are applied to specific ranges of the iris image. Due to variability of the amplitude information, which is heavily dependent on external factors such as image contrast, illumination and camera gain, only the complex-valued phase information is used in the matching process [39]. The phase information is quantized into four quadrants of the complex plane which results in a complex bit whose real and complex component will have a value of either 0 or 1 (Figure 2.8$)^{1}$. The resulting values, or bits, compose a binary vector which is called the $\operatorname{IrisCode}{ }^{\circledR}$.

To determine the level of statistical significance between two IrisCodes ${ }^{\circledR}$, Hamming distance is used. The Hamming distance is the measurement of the number of bits between two templates which are not the same. The resulting value is the match score between the templates. Hence the Hamming distance is a dissimilarity score and the lower the score between two templates the more likely they are from the same user. Ideally, the Hamming distance between two images of the same user would be 0 , but due to occlusion and other uncontrollable factors (intra-class variations), the genuine scores will have some dissimilar bits. The Hamming distance (HD) is defined as follows:

$$
H D=\frac{\left\|I_{1} \otimes I_{2} \bigcap M_{1} \bigcap M_{2}\right\|}{M_{1} \bigcap M_{2}} .
$$

where $I_{1}$ and $I_{2}$ are the IrisCodes ${ }^{\circledR}$ of the two iris images, and $M_{1}$ and $M_{2}$ are their respective masks. The masks, which are generated during the segmentation process, identify portions of the IrisCodes ${ }^{\circledR}$ that conforms to non-iris noise (sclera, eyelashes, eyelids, etc.). The logical operator XOR $(\otimes)$ detects disagreement between any corresponding bits in the $\operatorname{IrisCode}^{\circledR}$, while the AND $(\bigcap)$ operator ensures that the bits in question both correspond to this iris texture. The norm operator $(\|\|)$ computes the norm of the bit vector.

To observe the general performance of Gabor filtering, the performance of each channel prior to fusion is shown in Figure 2.9. A receiver operating characteristic(ROC) curve is used as a graphical representation of matching performance.

\footnotetext{
${ }^{1}$ http://getwww.uni-paderborn.de/
} 

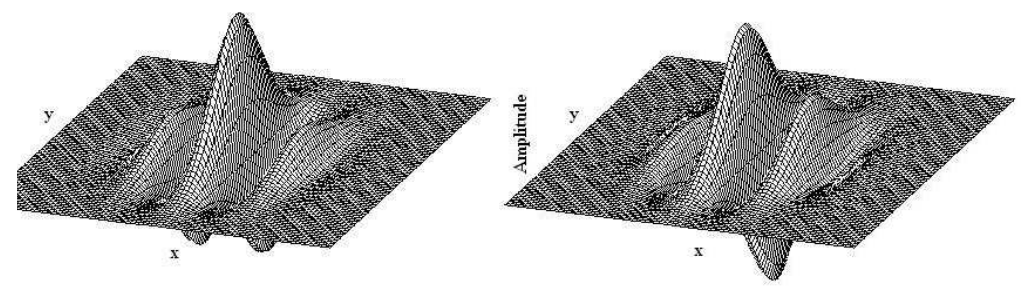

Figure 2.8: Three dimensional plots of the real (left) and imaginary (right) parts of the Gabor filter.

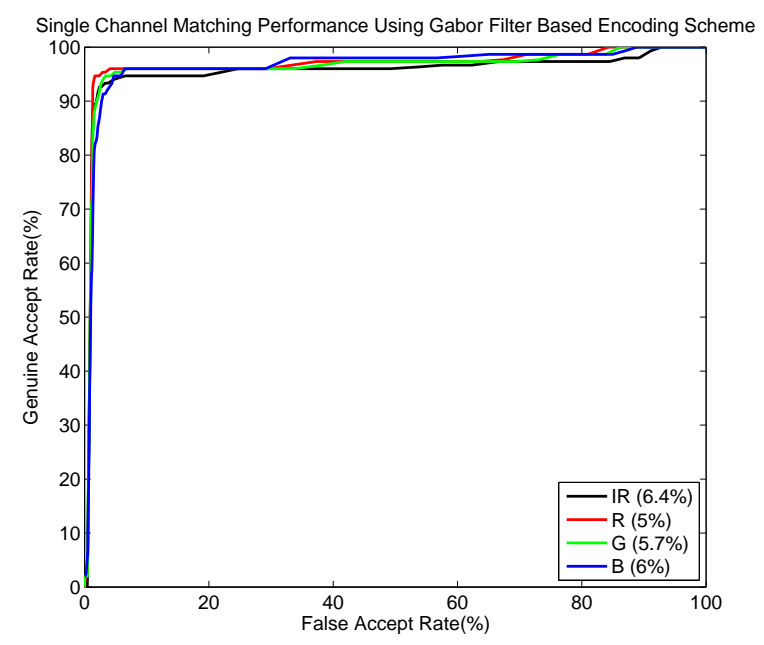

Figure 2.9: Matching performance using the Gabor based encoding scheme. The ROC curve shows the performance of each channel independently when compared against all other images from the same channel across the whole multispectral database. 


\subsection{Score Level Fusion}

To improve matching performance and fully benefit from the additional information provided by multispectral data, score level fusion is applied. A dynamic fusion rule is described which merges the matching score from only two of the four channels based on the textural quality and image characteristics of each channel.

Due to variations in eye color, the performance of each channel varies across users. Early analysis shows that dark brown, light brown and green eyes generally perform the best in the infrared and red channels, while blue eyes show the best performance in the green and blue channels (Figure 2.10). This simple manual classification of eye color and utilization of specific channels, does lead to higher matching performance compared to score level fusion across all four channels. Based on this initial analysis, users were divided into classes based on their perceived eye color. Classification was performed through visual inspection of the color image. Users were assigned into one of the three classes: dark brown, light brown/green, and blue. Though these three color classes do not encompass the complete spectra of all possible eye colors, the remaining colors (hazel, gray, amber, etc) are expected to fall within one of the three classes based on similarities in chromaticity. Two benchmark performance curves are generated by using the fusion rule based on visual inspection of the user classes. A visual test of texture quality is used where each band of a multispectral image is visually inspected to rank the amount and quality present. The test is performed for multiple images of each eye color class. The bands of each image which show the best quality were chosen for score level fusion. The benchmark for fusion using the "sum" rule is defined as

$$
\begin{aligned}
S_{b r w n} & =\frac{I R_{b r w n}+R_{b r w n}}{2}, \\
S_{l b g} & =\frac{I R_{l b g}+R_{l b g}}{2}, \\
S_{\text {blue }} & =\frac{G_{b l u e}+B_{b l u e}}{2} .
\end{aligned}
$$

where $S_{b r w n}, S_{l b g}$, and $S_{b l u e}$ are the scores for the default sum fusion rule for the dark brown, 
light brown/green, and blue eyes, respectively. Thus, the matching scores pertaining to an eye are defined by its color class and channel. Therefore, for example, $I R_{b r w n}$ and $R_{b r w n}$ represent the IR and red channels of irides of the brown color class.

Further analysis shows that simply classifying each eye based on its perceived eye color does not provide the optimal fusion scheme. Due to variations in the interpretation of eye color classification, human perception is not a precise classification scheme due to the lack of a measurable quantitative feature. Furthermore, using only three classes may be too restrictive to properly classify all possible eye colors. By forcing users into a single class when they have features which may conform to multiple classes, can lead to poor fusion performance. The constrained fusion rules which the benchmark fusion implements, also limits performance by suggesting that the two bands of the multispectral image which are chosen for each eye color class are the optimal bands for all users of that class.

To alleviate these problems a more advanced dynamic fusion rule is applied. In this experiment the notion of predefined eye classes has been removed and each image is classified based on it's textural characteristics. Each channel of the multispectral iris template is first analyzed using measures of contrast and texture. Six measures of texture are used to analyze the intensity profile of each band: mean, standard deviation, smoothness, third moment, uniformity and entropy (Table 2.1) [42]. Each textural measure provides a distinct analysis of the textural information of the image allowing for up to six unique fusion rules. After the original iris image is segmented and unwrapped, the four channels of the image are then analyzed individually. Each channel is ranked based on the quality of texture across the iris. An overall ranking of texture is defined as the average ranking across all six measurements (Figure 2.11). Let $R_{1}$ and $R_{2}$ be the rank one and rank two channels, respectively.

$$
R_{1}=\max \left(\operatorname{mean}\left(T M_{\mu}(i), T M_{\text {std }}(i), T M_{\text {smth }}(i), T M_{3 r d}(i), T M_{\text {uni }}(i), T M_{\text {ent }}(i)\right)\right) .
$$




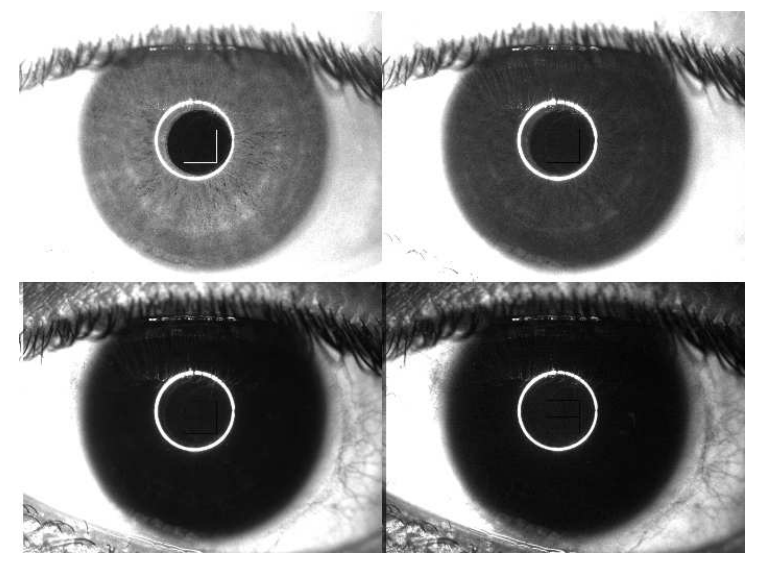

(a) Brown Eye

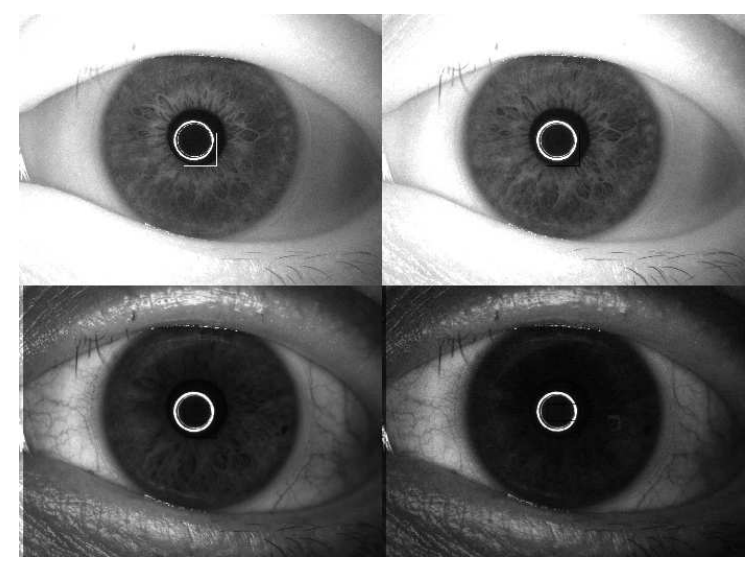

(b) Light Brown/Green

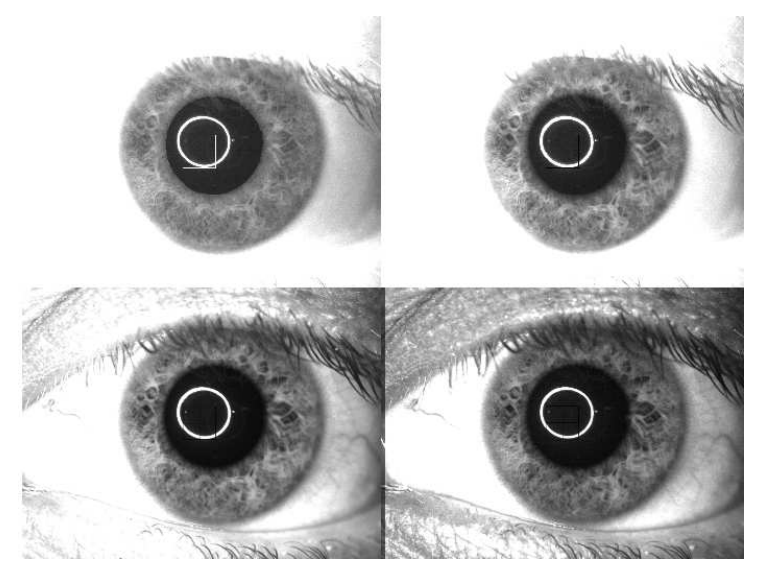

(c) Blue Eye

Figure 2.10: Comparing the imaging qualities of the four channel images for the three classes of eye colors. Based on the eye color, levels of information are present in each channel. Starting from the top right and going clockwise the channels are red, green, blue and IR. 


$$
R_{2}=\max \left(\operatorname{mean}\left(T M_{\mu}(i), T M_{s t d}(i), T M_{s m t h}(i), T M_{3 r d}(i), T M_{u n i}(i), T M_{\text {ent }}(i)\right)\right) \bigcap R_{2} \neq R_{1}
$$

Here the texture measures are denoted by $T M_{\mu}(i), T M_{s t d}(i), T M_{s m t h}(i), T M_{3 r d}(i), T M_{u n i}(i)$ and $T M_{\text {ent }}(i)$ which represent the mean, standard deviation, smoothness, third moment, uniformity and entropy, respectively. The variable $i$ defines the current band of the multispectral image. The two channels which rank first and second are then chosen for score level fusion during matching stage. The final score level fusion is performed using the sum rule:

$$
S_{d y n}=\frac{I_{1}+I_{2}}{2}
$$

where $S_{d y n}$ is the dynamic score level sum rule. The variables $I_{1}$ and $I_{2}$ represent the matching scores from the two channels of the multispectral image which rank the highest based on the measurements of texture. By determining the channels of the image which will be subjected to matching prior to encoding, the processing requirements can be essentially halved since only the two highest ranking channels, based on texture measurements, need to be further processed.

\begin{tabular}{|l|c|c|}
\hline Moment & Expression & Measure of Texture \\
\hline Mean & $m=\sum_{i=0}^{L-1} z_{i} p\left(z_{i}\right)$ & Measurement of average intensity. \\
\hline Standard Deviation & $\sigma=\sqrt{\mu_{2}(z)}$ & Measurement of average contrast. \\
\hline Smoothness & $R=1-\frac{1}{1+\sigma^{2}}$ & $\begin{array}{c}\text { Measurement of relative intensity smoothness } \\
\text { across the image. }\end{array}$ \\
\hline Third Moment & $\mu_{3}=\sum_{i=0}^{L-1}\left(z_{i}-m\right)^{3} p\left(z_{i}\right)$ & $\begin{array}{c}\text { Measurement of skewness of the image } \\
\text { histogram. }\end{array}$ \\
\hline Uniformity & $U=\sum_{i=0}^{L-1} p^{2}\left(z_{i}\right)$ & $\begin{array}{c}\text { Measurement of uniformity across the } \\
\text { image. }\end{array}$ \\
\hline Entropy & $e=-\sum_{i=0}^{L-1} p\left(z_{i}\right) \log _{2} p\left(z_{i}\right)$ & $\begin{array}{c}\text { Measurement of randomness across the } \\
\text { image. }\end{array}$ \\
\hline
\end{tabular}

Table 2.1: Measures of image texture used by the dynamic fusion algorithm. Let $z_{i}$ be the location of a single pixel in the Cartesian plane of an image. 


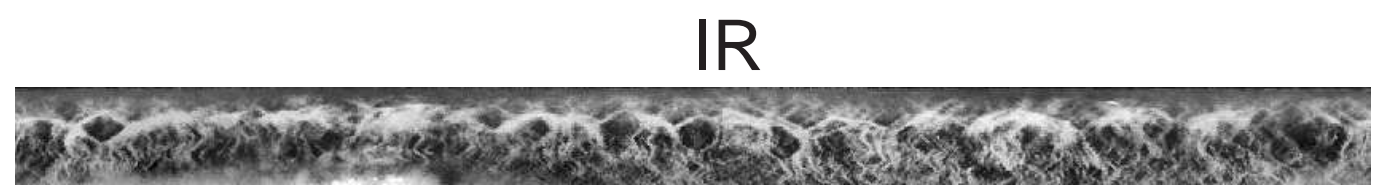

Average Intensity $=115.0706$ Average Contrast $=48.7727 \quad R=.0353$

Third Moment $=.6337$ Uniformity $=.0058$ Entropy $=7.5575$

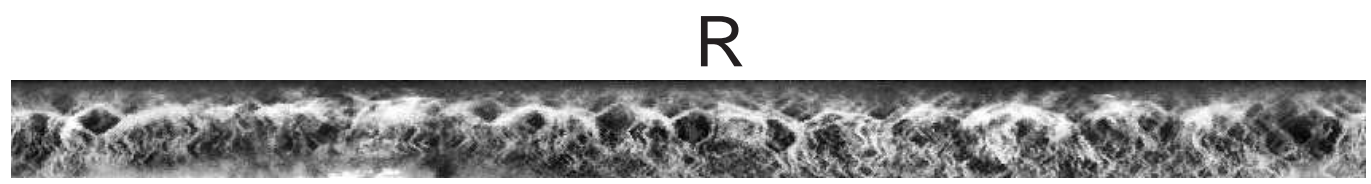

Average Intensity $=117.1390 \quad$ Average Contrast $=57.0086 \quad R=.0477$

Third Moment $=1.1909$ Uniformity $=.0050$ Entropy $=7.7633$

$\mathrm{G}$

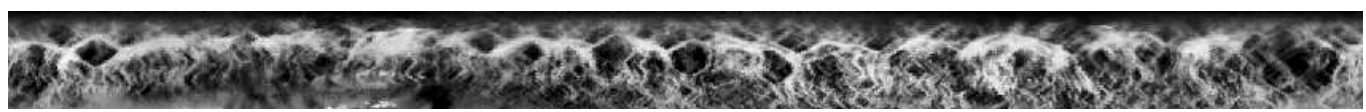

Average Intensity $=96.1115$ Average Contrast $=57.6967 \quad R=.0487$

Third Moment $=1.2462$ Uniformity $=.0052$ Entropy $=7.7223$

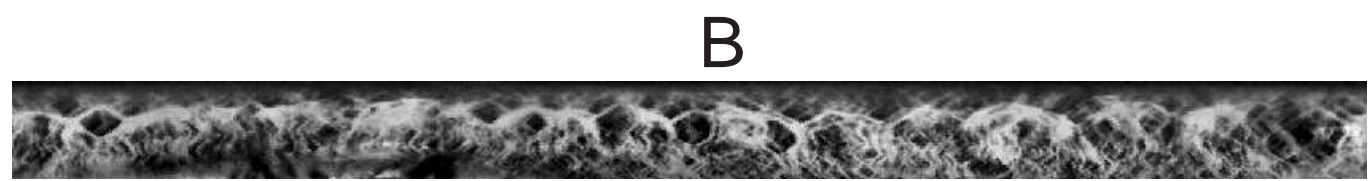

Average Intensity $=92.0740 \quad$ Average Contrast $=53.7488 \quad R=.0425$

Third Moment $=1.0226$ Uniformity $=.0055$ Entropy $=7.6325$

Figure 2.11: Four channels of a multispectral normalized blue iris image. For all measurements except uniformity as the amount/quality of texture increases in the image the score also increases. On the contrary, an image with high levels of texture will have a low uniformity score. 


\subsubsection{Dynamic Score Level Fusion Results for Gabor Based Texture Encoding}

Within the constraints of a limited database, the results of the dynamic score level fusion show a potential increase in performance when compared to the matching performance of the individual channels (Figures 2.13, 2.12 and Table 2.2). The resulting performance may be biased due to the limited data available. No other known databases of multispectral iris images captured in the wavelengths required for this work are currently available. To further examine the performance of the fusion system, the UBIRIS color iris databases were utilized. Since the UBIRIS database consist of only three channels (red, green and blue), the resulting performance is not necessarily conclusive in predicting the dynamic fusion algorithm across a four channel multispectral image. The intention of incorporating a three band image database is to determine the potential of applying the dynamic fusion algorithm to images lacking the near-IR band and determine its impact on performance.

\begin{tabular}{|l|c|c|c|c|c|c|}
\hline Color Class & IR & R & G & B & Baseline Fusion & Dynamic Fusion \\
\hline Brown & $2.7 \%$ & $1.7 \%$ & $2.2 \%$ & $4.4 \%$ & $1.5 \%$ & $2.6 \%$ \\
\hline L. Brown/Green & $5.7 \%$ & $5.7 \%$ & $5.7 \%$ & $5.7 \%$ & $5.7 \%$ & $5.7 \%$ \\
\hline Blue & $13.4 \%$ & $9.4 \%$ & $9.3 \%$ & $9.4 \%$ & $9.4 \%$ & $9.4 \%$ \\
\hline Database & $6.4 \%$ & $5 \%$ & $5.7 \%$ & $6 \%$ & $5.9 \%$ & $5 \%$ \\
\hline
\end{tabular}

Table 2.2: The EER for dynamic fusion when using the Gabor based encoding scheme.

Recognition and fusion is performed on images from UBIRIS database in the same manner as described for the multispectral images. Fusion is only slightly altered since the IR channel is not present. The fusion algorithm now, is required to choose from three channels instead of four. Performance is measured with an ROC curve and the equal error rate (EER) (Figure 2.14 and Table 2.3).

Fusion performance in color images shows the strength that the near-IR band has in providing an ideal iris template, especially for brown and light brown eyes. With the absence of the near-IR 


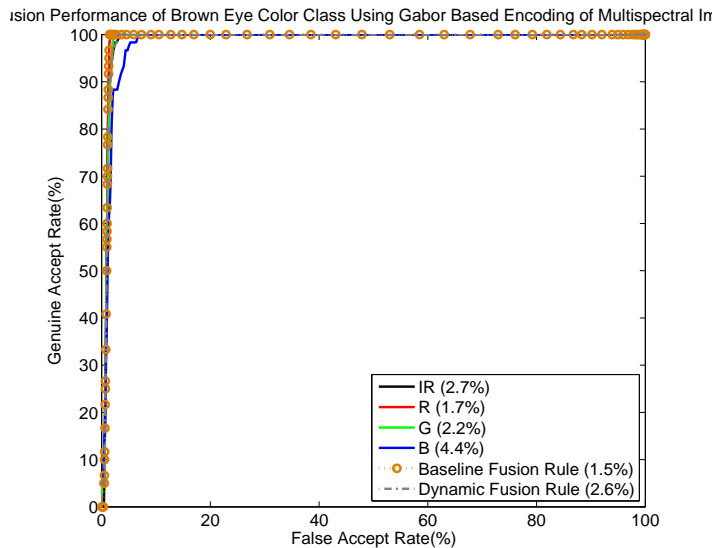

(a) Brown Eye Color Class

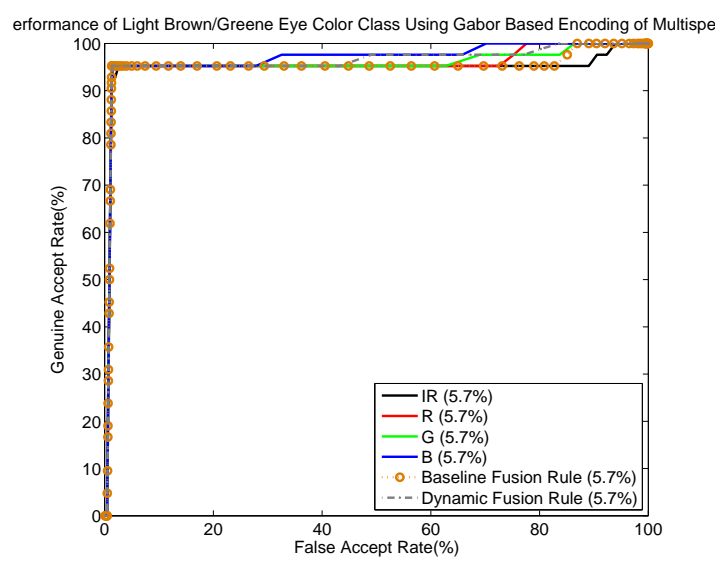

(b) Light Brown/Green Eye Color Class

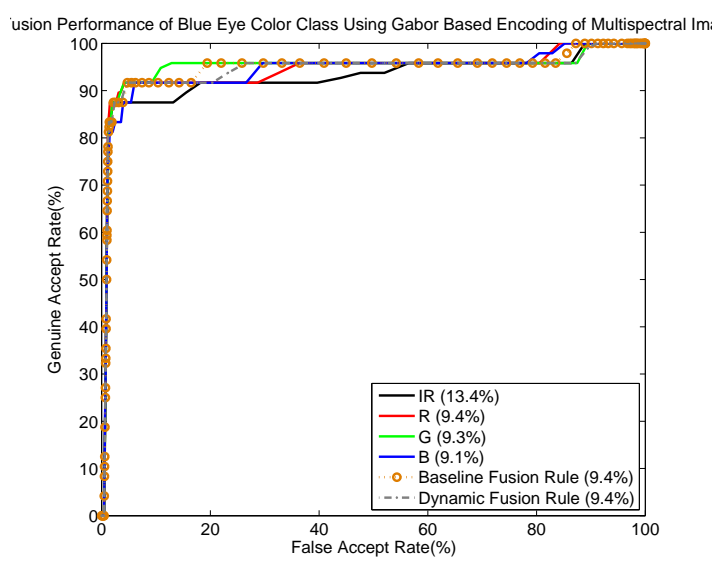

(c) Blue Eye Color Class

Figure 2.12: Performance curves for eye color class when the dynamic fusion rule is applied to only users of each color class independently. 


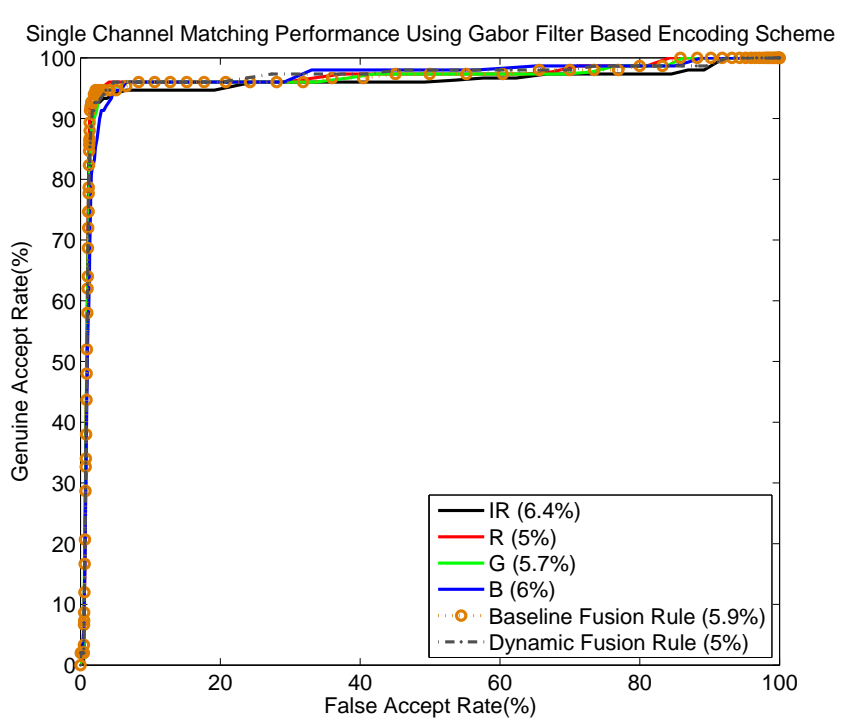

Figure 2.13: Matching results of the dynamic fusion algorithm versus the baseline fusion algorithm and the individual channel performance when applied to the multispectral image database.

\begin{tabular}{|l|c|c|c|c|}
\hline Color Class & $\mathbf{R}$ & $\mathbf{G}$ & $\mathbf{B}$ & Dynamic Fusion \\
\hline Brown & $16 \%$ & $17.8 \%$ & $17.7 \%$ & $17.1 \%$ \\
\hline L. Brown/Green & $11.6 \%$ & $11.7 \%$ & $12.2 \%$ & $14.9 \%$ \\
\hline Blue & $10.9 \%$ & $10.6 \%$ & $10.9 \%$ & $10.4 \%$ \\
\hline Database & $11.6 \%$ & $11.7 \%$ & $12.2 \%$ & $14.9 \%$ \\
\hline
\end{tabular}

Table 2.3: The EER for Dynamic fusion when using Gabor based encoding scheme on color iris images. 


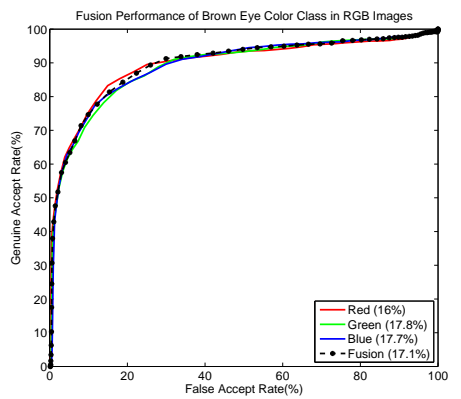

(a) Brown Eye Color Class

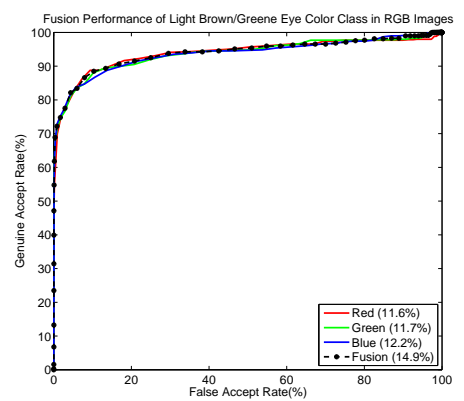

(b) Light Brown/Green Eye Color Class

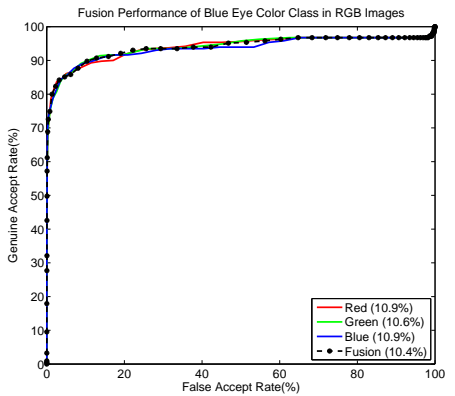

(c) Blue Eye Color Class

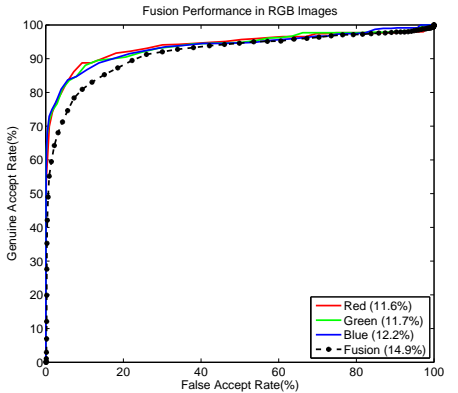

(d) Total UBIRIS Database and Fusion

Figure 2.14: Dynamic score level fusion performance on color images. 
channel the fusion performance declined across all eye color classes insinuating that the near-IR channel is essential to ensure high performance and, when compared to color images in the sRGB color space, performs better across eyes of all colors than a combination of the color bands.

\subsection{Local Binary Patterns}

Local binary patterns (LBP) is a gray-scale invariant textural analysis operator for both color and gray-scale. The LBP defines the variability in pixel intensity in a local block shaped neighborhood. In it's early implementations, the operator worked on a $3 \times 3$ pixel block, where the eight neighboring pixels around the center pixel are thresholded based on the value of the central pixel $[43,44]$. In this work, an iris encoding scheme is developed based on this early implementation of LBP texture analysis. The resulting values of the threshold operation create a unique binary code for each pixel. LBP texture analysis and the Gabor based encoding scheme are applied following image segmentation, unwrapping and normalization as described previously in this chapter. The LBP operation is defined as

$$
L B P_{c}(p)=s\left(g_{p}-g_{c}\right)
$$

where $L B P_{c}(p)$ is the textural response for the central pixel $\left(g_{c}\right)$ of the segmented iris image (Figure 2.15). The value $p$ is the current neighbor pixel which varies from 0 to $P-1$, where $P$ is the number of neighborhood pixels evaluated for each central pixel. Since a textural response is calculated for each pixel $p$, a binary string will be stored for each central pixel $c$, resulting in a 3D binary code template. $g_{p}$ is the pixel intensity of the current neighbor pixel. The neighborhood is defined to be the pixels along the parameter of the filter. If the filter size is greater than $3 \times 3$ then there will be pixels inside the neighborhood which do not fall along the edge of the filter. These pixels are ignored (Figure 2.15). Since the difference between pixels is affected by scaling, only the sign of the differences between the two pixels is considered. Thus, the function $s$ is 
defined as

$$
s(x)= \begin{cases}1, & \text { if } x \geq 0, \\ 0, & \text { if } x<0 .\end{cases}
$$

which retains only the sign of the variable $x$. The LBP analysis is applied iteratively where the LBP center pixel $g(c)$ is moved across the image one pixel at a time ensuring that a LBP value is derived for each pixel within the image. Each channel of the multispectral image is encoded independently resulting in four 3D LBP codes per image.

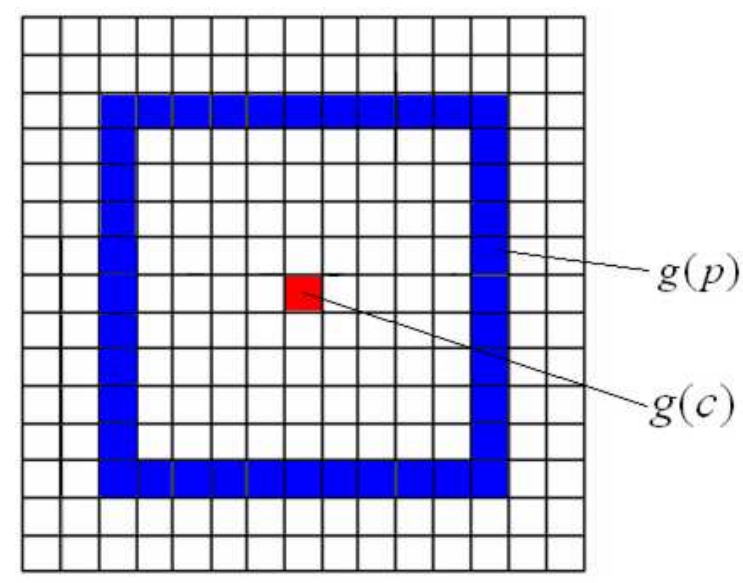

Figure 2.15: Graphical representation of a 11x11 LBP neighborhood. The pixels which are colored blue are the only pixels within the neighborhood that are considered.

Like the IrisCode ${ }^{\circledR}$, the 3D binary template created from LBP texture analysis allows for a simple and fast matching algorithm. A matching score is determined by applying a three dimensional logical XOR operator to the two templates subjected to matching. A matching score is determined by calculating the Hamming distance between the two templates. 


\subsubsection{Dynamic Score Level Fusion Based on Local Binary Pattern Matching Scores}

Overall fusion and single-band matching performance derived from LBP encoding shows poor performance. Though the performance in general shows little promise it is important to note the increase in performance when the dynamic fusion rule is applied compared to both singleband performance as well as the baseline fusion performance(Figure 2.16). The baseline fusion rule is implemented as discussed earlier (see Score Level Fusion). To determine the effect eye color has on performance, results are first broken down by eye color. The eye color is that of the known template being compared against other templates. The known user is compared against all templates in the database. For both dark brown eye and blue eyes, the default as well as dynamic fusion rule show a significant increase in performance. Since both fusion rules are designed to illicit the optimal performance in the visible spectrum they both make excellent candidates for multispectral fusion at the score level. Though recognition performance of both fusion algorithms remain constant for light brown/green eyes it is much lower than the performance witnessed from single-band matching. The high matching performance, compared to the other eye color classes, may simply be due to the small data set which was used for this experiment. Overall the data set was not uniformly distributed across the three eye color classes and was only limited to sixteen unique light brown/green eyes. Figure 2.17 shows the overall fusion performance when comparing the baseline fusion rule with the dynamic fusion rule. The six measures of texture described before were used independently and show a varying degree of performance. Each of the six fusion rules based on the textural measures have a lower performance than the predefined fusion rule. The slight decrease in performance of the default fusion rule and the dynamic fusion rules may be contributed to the small data set. Even considering the decrease in performance a dynamic fusion rule based on only a single texture measure still shows an overall increase in performance compared to individual channel matching. 


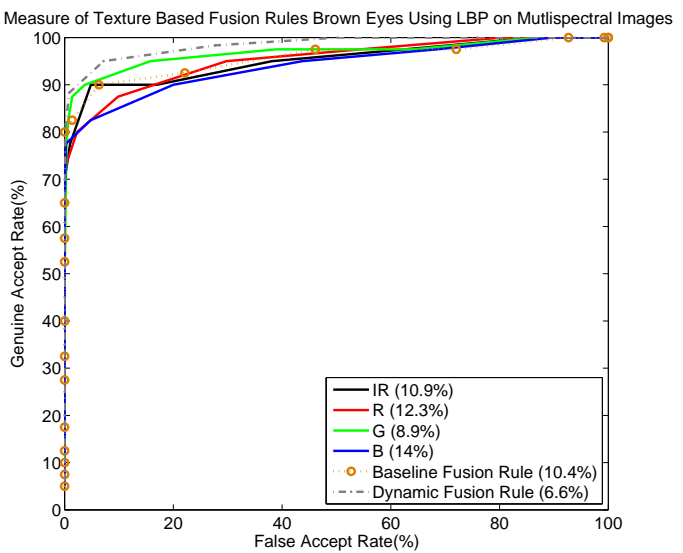

(a) Brown Eye Color Class

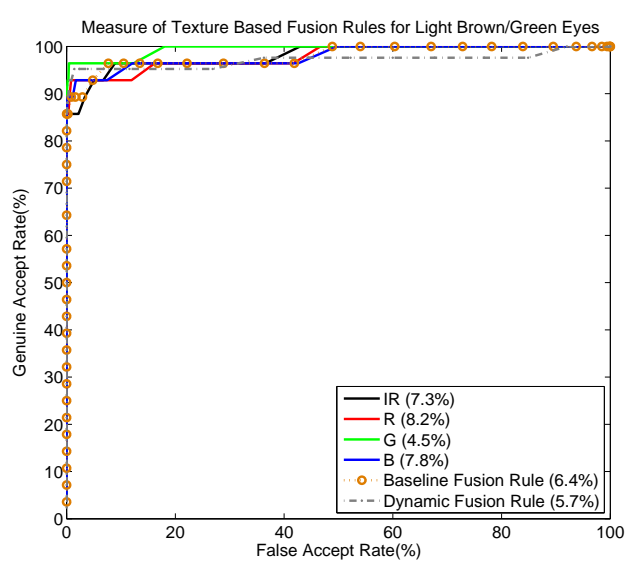

(b) Light Brown/Green Eye Color Class

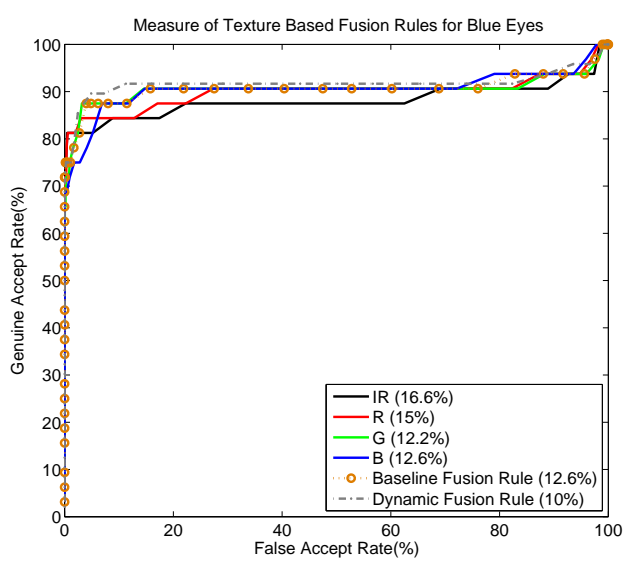

(c) Blue Eye Color Class

Figure 2.16: The ROC curves demonstrating the single-band matching performance as well as the default fusion rule and dynamic fusion rule. Users are classified by eye color to emphasize the role color has on matching performance in the visible spectrum. 


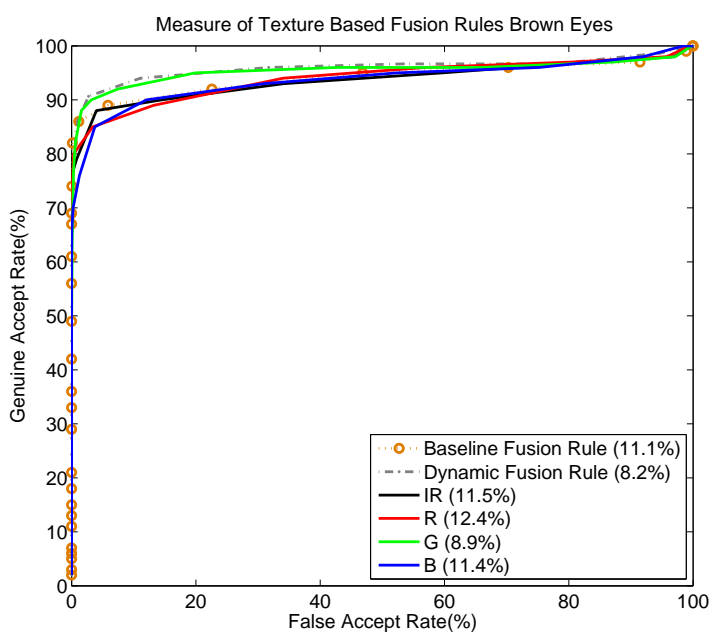

Figure 2.17: ROC curve for the entire database encoded using LBP. Performance curves are shown for each band of the image as well as the baseline and dynamic fusion performance.

\begin{tabular}{|l|c|c|c|c|c|c|}
\hline Color Class & IR & R & G & B & Baseline Fusion & Dynamic Fusion \\
\hline Brown & $10.9 \%$ & $12.3 \%$ & $8.9 \%$ & $14 \%$ & $10.4 \%$ & $6.6 \%$ \\
\hline L. Brown/Green & $7.3 \%$ & $8.2 \%$ & $4.5 \%$ & $7.8 \%$ & $6.4 \%$ & $5.7 \%$ \\
\hline Blue & $16.6 \%$ & $15 \%$ & $12.2 \%$ & $12.6 \%$ & $12.6 \%$ & $10 \%$ \\
\hline Database & $11.5 \%$ & $12.4 \%$ & $8.9 \%$ & $11.4 \%$ & $11.1 \%$ & $8.2 \%$ \\
\hline
\end{tabular}

Table 2.4: The EER for Dynamic fusion when using LBP based encoding scheme. 


\subsection{Summary}

In this chapter a score level fusion algorithm for multispectral and color iris images was introduced. The algorithm was used for scores generated with both Gabor based and LBP texture encoding. The results and performance were widely varied and telling of potential of using the visible spectrum for texture-based iris recognition. When analyzing the results of both the multispectral and color databases, when the Gabor based encoding was applied we saw an insignificant increase in performance and in some cases a decrease in performance. Under further analysis we see that the worst performance appears in the color data set where the near-IR component of the image is not present. As discussed earlier in this chapter in the visible spectrum the textural quality of certain bands can be very poor. This is most prominent in dark brown eyes. This would lead to poor encoding results within the respective bands. Since the dynamic fusion algorithm is required to fuse two channels, often scores from low quality templates were included into the final fusion score simply because they were the best available data. In future versions of the dynamic score level fusion algorithm a measure of quality must be incorporated to ensure that only score generated from templates of sufficient quality are included for fusion. Leading to cases such as what was seen with the UBIRIS data set where texture quality is so

poor in certain bands (green and blue bands of dark brown eyes) that a single score (red channel of dark brown eye) may be better.

Besides enforcing the notion that near-IR is the ideal imaging band for dark colored eyes the results of this work may be an indication that texture-based encoding is not ideal for images captured in the visible sRGB color space. Previous work by Boyce et al. [18] has shown results where recognition performance within the visible color spectrum using intensity based encoding out performs traditional texture-based encoding. To fully understand the capabilities of the fusion algorithms other, non-texture-based encoding schemes such as the one proposed by Boyce et al. must be utilized. 


\section{Chapter 3}

\section{Iris Color and Color Space Analysis}

\subsection{Motivation}

By including the information in the visible spectrum into iris recognition, the unique characteristics that are exclusive to each eye color have become relevant to recognition performance [45]. Analysis using the visible spectrum has shown that the matching performance per channel varies heavily in the traditional sRGB color space based on the color of the user's eye. The impact color has on matching performance has lead us to further analyze the representation of color through various color spaces to try to elicit additional information from the image that can be used to increase matching performance. The goal is to determine if an optimal color space for both multispectral and color iris matching exists. As in face recognition, where the YCbCr color space has shown to be a viable alternative to the sRGB [46], it is the intention of this work to determine the underlying characteristics of a color space which will lead to optimum multispectral iris matching performance. 


\subsection{Color Space Transformations}

To ensure that all aspects of both image luminosity and chromaticity were included in the analysis, a vast number of color space transformations were chosen. Each transform was analyzed based on general channel-to-channel matching performance and the correlation of performance between image channels. Below is a mathematical description of the color space transformations used in our work. In the work the following color spaces are explored: sRGB, CIE Lab, YCbCr, HSV and CMYK.

It is not possible to convert directly from the sRGB to the CIE Lab color space. An intermediate step of first transforming from sRGB to CIE XYZ must first be implemented, which can then be used to convert to the CIE Lab color space. Let $[R G B]$ represent the components of the sRGB image and $[r g b]$ be the normalized RGB components. The normalization of the RGB components is described below ${ }^{1}$ :

$$
\begin{aligned}
& r= \begin{cases}R / 12.92, & R \leq 0.04045, \\
\left(\frac{R+0.055}{1.055}\right)^{2.4}, & R>0.04045,\end{cases} \\
& g= \begin{cases}G / 12.92, & G \leq 0.04045, \\
\left(\frac{G+0.055}{1.055}\right)^{2.4}, & G>0.04045,\end{cases} \\
& b= \begin{cases}B / 12.92, & B \leq 0.04045, \\
\left(\frac{B+0.055}{1.055}\right)^{2.4}, & B>0.04045 .\end{cases}
\end{aligned}
$$

The $r, g$, and $b$ values are normalized using primaries derived from the colorimetric characteristics of the sRGB color space. Next, the CIE XYZ transformation can be applied as

$$
[X Y Z]=[r g b][M],
$$

\footnotetext{
${ }^{1}$ CIE Lab Color space transform equations are courtesy of Bruce Lindbloom and can be found at http://brucelindbloom.com/
} 
where $[X Y Z]$ are the components of the CIE XYZ image and $M$ is the transformation matrix calculated from the RGB reference primaries ${ }^{2}$. Let $\left(x_{r}, y_{r}\right),\left(x_{g}, y_{g}\right)\left(x_{b}, y_{b}\right)$ represent the chromaticity coordinates and $\left(X_{W}, Y_{W}, Z_{W}\right)$ be the coordinates of the white point of the RGB system. From this the following values are calculated:

$$
\begin{gathered}
X_{r}=x_{r} / y_{r}, \\
Y_{r}=1, \\
Z_{r}=\left(1-x_{r}-y_{r}\right) / y_{r}, \\
X_{g}=x_{g} / y_{g}, \\
Y_{g}=1, \\
Z_{g}=\left(1-x_{g}-y_{g}\right) / y_{g}, \\
X_{b}=x_{b} / y_{b}, \\
Y_{b}=1, \\
\end{gathered}
$$

\footnotetext{
${ }^{2}$ Reference primaries define the colorimetric specifications of the color space, values such as gamma, white point, and color efficiency. A detailed list of colorimetric characteristics for the RGB family of color spaces can be found at http://brucelindbloom.com/.
} 


$$
\left[S_{r} S_{g} S_{b}\right]=\left[X_{W} Y_{W} Z_{W}\right]\left[\begin{array}{ccc}
X_{r} & Y_{r} & Z_{r} \\
X_{g} & Y_{g} & Z_{g} \\
X_{b} & Y_{b} & Z_{b}
\end{array}\right]^{-1}
$$

From these intermediate values, the transformation matrix is calculated as:

$$
M=\left[\begin{array}{ccc}
S_{r} X_{r} & S_{r} Y_{r} & S_{r} Z_{r} \\
S_{g} X_{g} & S_{g} Y_{g} & S_{g} Z_{g} \\
S_{b} X_{b} & S_{b} Y_{b} & S_{b} Z_{b}
\end{array}\right] .
$$

Now that the conversion to CIE XYZ is complete, the final transformation to CIE Lab can commence. Let $[L a b]$ be the channels of the CIE Lab image, where

$$
\begin{aligned}
& L=116 f_{y}-16, \\
& a=500\left(f_{x}-f_{y}\right), \\
& b=200\left(f_{y}-f_{z}\right),
\end{aligned}
$$

and

$$
\begin{gathered}
f_{x}= \begin{cases}\sqrt[3]{x_{r}}, & x_{r}>\varepsilon, \\
\frac{\kappa x_{r}+16}{116}, & x_{r} \leq \varepsilon .\end{cases} \\
f_{y}= \begin{cases}\sqrt[3]{y_{r}}, & y_{r}>\varepsilon, \\
\frac{\kappa y_{r}+16}{116}, & y_{r} \leq \varepsilon .\end{cases} \\
f_{z}= \begin{cases}\sqrt[3]{z_{r}}, & z_{r}>\varepsilon, \\
\frac{\kappa z_{r}+16}{116}, & z_{r} \leq \varepsilon .\end{cases} \\
x_{r}=\frac{X}{X_{r}},
\end{gathered}
$$




$$
\begin{gathered}
y_{r}=\frac{Y}{Y_{r}}, \\
z_{r}=\frac{Z}{Z_{r}}, \\
\varepsilon= \begin{cases}0.008856, & \text { Actual CIE Standard, } \\
\frac{216}{24389}, & \text { Intent of the CIE Standard. }\end{cases} \\
\kappa= \begin{cases}903.3, & \text { Actual CIE Standard, } \\
\frac{24389}{27}, & \text { Intent of the CIE Standard. }\end{cases}
\end{gathered}
$$

where $X_{r}, Y_{r}$, and $Z_{r}$ are the reference to the white point in the CIE XYZ color space. In our calculations, the actual CIE standard for $\varepsilon$ and $\kappa$ were used instead of the intended standard.

The color space $\mathrm{YCbCr}$ is derived from the sRGB color space. Let the three channels of the YCbCr image be $[\mathrm{YCbCr}]$ and the channels of the sRGB be defined as $[R G B]^{3}$ :

$$
\begin{gathered}
Y^{\prime}=K_{r} R^{\prime}+\left(1-K_{r}-K_{b}\right) G^{\prime}+K_{b} * B, \\
C b=\frac{B^{\prime}-Y^{\prime}}{2\left(1-K_{b}\right)}, \\
C r=\frac{R^{\prime}-Y^{\prime}}{2\left(1-K_{r}\right)},
\end{gathered}
$$

where $K_{b}$ and $K_{r}$ are defined based on the corresponding RGB color space. For the transformations used in this thesis, the two values, $K_{b}$ and $K_{r}$, are defined based on the television color standard ITU-R BT.601, the standard for color of a standard-definition television ${ }^{4}$. Using this standard, the values of $K_{b}$ and $K_{r}$ are defined as 0.114 and 0.299 , respectively. The prime symbol $(')$ indicates that the values are gamma corrected and normalized between $0-1$.

The color space HSV is obtained directly from sRGB. Let MAX be the maximum value of the three intensity values of a sRGB triplet at a given pixel, and MIN be the minimum of $[R G B]$

\footnotetext{
${ }^{3}$ http://www.poynton.com/PDFs/ColorFAQ.pdf

${ }^{4}$ http://www.itu.int/net/home/index.aspx
} 
at the same pixel. The HSV color space is defined at each pixel location by the triplet $[H S V]$, and is calculated as follows:

$$
\begin{aligned}
& H= \begin{cases}\text { undefined, } & M A X=M I N, \\
60^{\circ} \frac{G-B}{M A X-M I N}+0^{\circ}, & M A X=R \text { and } G \geq B, \\
60^{\circ} \frac{G-B}{M A X-M I N}+360^{\circ}, & M A X=R \text { and } G<B, \\
60^{\circ} \frac{B-R}{M A X-M I N}+120^{\circ}, & M A X=G, \\
60^{\circ} \frac{R-G}{M A X-M I N}+240^{\circ}, & M A X=B .\end{cases} \\
& S= \begin{cases}0, & M A X=0 \\
1-\frac{M I N}{M A X}, & \text { otherwise }\end{cases} \\
& V=M A X .
\end{aligned}
$$

The CMYK color space is also converted directly from the sRGB color space. Unlike other color spaces, a fourth channel is included in this color space that measures the black intensity exclusively. CMYK cannot be directly transformed from the sRGB color space, but has to be first transformed to the intermediate CMY space. Let $\left[C^{\prime} M^{\prime} Y^{\prime}\right]$ be the three components of the intermediate CMYK transformation [47]:

$$
\begin{aligned}
& C^{\prime}=1-R, \\
& M^{\prime}=1-G, \\
& Y^{\prime}=1-B,
\end{aligned}
$$

First, the value $K_{v a r}$ must be determined. $K_{v a r}$ is an intermediate value used to calculate the black component of the CMYK color space. Once $K_{v a r}$ is calculated the four channels of the

\footnotetext{
${ }^{5}$ http://www.cs.rit.edu/ncs/color/t_convert.html
} 
CMYK can be calculated.

$$
\begin{gathered}
K_{\text {var }}= \begin{cases}C^{\prime}, & C^{\prime}<K_{\text {var }}, \\
M^{\prime}, & M^{\prime}<K_{\text {var }}, \\
Y^{\prime}, & Y^{\prime}<K_{\text {var }}, \\
1, & \text { otherwise. }\end{cases} \\
{[C M Y K]= \begin{cases}{[0001],} & K_{\text {var }}=1, \\
{\left[\frac{C^{\prime}-K_{\text {var }}}{1-K_{\text {var }}} \frac{M^{\prime}-K_{\text {var }}}{1-K_{\text {var }}} \frac{Y^{\prime}-K_{\text {var }}}{1-K_{\text {var }}} K_{\text {var }}\right],} & \text { otherwise. }\end{cases} }
\end{gathered}
$$

Here $[C M Y K]$ is a quadruplet at a single pixel in the CMYK color space.

\subsection{Image Processing and Color Space Adaption}

In order to convert between the original color space of sRGB and one of the color spaces used for analysis, the irides were first segmented and normalized. Using the same preprocess, segmentation and normalization processes as described in the pervious chapter, a sRGB color iris template is utilized as the baseline image on which all color space transforms are applied. Since we are only concerned with the transformation of the visible spectrum of the iris image, the near-IR component is omitted. The normalized template is then subjected to a color space transform. Once the transform is complete, encoding and matching is performed on each channel of the color image independently. Like the other components of recognition, the matching and encoding algorithms are as described in the second chapter. For all color space analysis, the Gabor based texture encoding was applied.

\subsection{Texture Enhancement}

To ensure optimum matching performance, image preprocessing calls for some type of contrast enhancement. In an iris recognition system, techniques such as histogram equalization $[48,49]$ and Perona-Malik anisotropic diffusion [50] are commonly used. Both techniques are effective 
at sharpening edges and increasing contrast in grayscale images. Applied to RGB color images, techniques such as these not only increase the contrast of the image but also alter the chromaticity. Since both the luminosity and chromaticity of the image are represented on the same component in the RGB color model, it is not possible to increase the luminosity without affecting the chromaticity. In order to ensure that the chromaticity of the image is not affected, the luminosity aspect of the image must be first isolated before enhancement. In an attempt to mimic the natural perception of the eye many color spaces separate the luminosity from the chromaticity [51]. In this work we examine the CIE Lab, YCbCR and HSV color space to determine which color space provides the best contrast enhancement while retaining the original chromaticity as closely as possible. Both the CIE Lab and YCbCr color space isolate the chromaticity completely.

The Luminance and Luma are contained completely on the $\mathbf{L}$ and $\mathbf{Y}$ channels of the CIE Lab and $\mathrm{YCbCr}$ color spaces, respectively. By applying histogram equalization to these channels exclusively, the contrast of the image can be increased without altering the chromatic characteristics. For enhancement in the HSV color space the value $(\mathbf{V})$ component is isolated and histogram equalization is performed. The $\mathbf{V}$ component of the HSV color space does not completely isolate chromaticity from luminosity, and, therefore, changes in chromaticity may be seen once enhancement is complete. For all three color space enhancements, once histogram equalization is complete the image is transformed back into the sRGB color space for further processing (Figure 3.1).

\subsubsection{Textural Quality in Enhanced Iris Templates}

As a preliminary test of enhancement, performance contrast of the iris template is measured using a six component measurement index. The components of the measurement index for textural quality are the same that are used for dynamic score level fusion (see Chapter 2). Table 3.1 compares contrast results of the original sRGB template with those of the sRGB templates after CIE Lab, YCbCr, and HSV histogram equalization.

All three enhancement algorithms demonstrate higher measures of contrast across all com- 


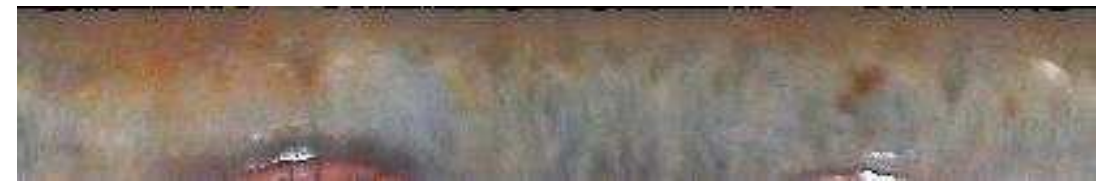

(a) Original sRGB image.

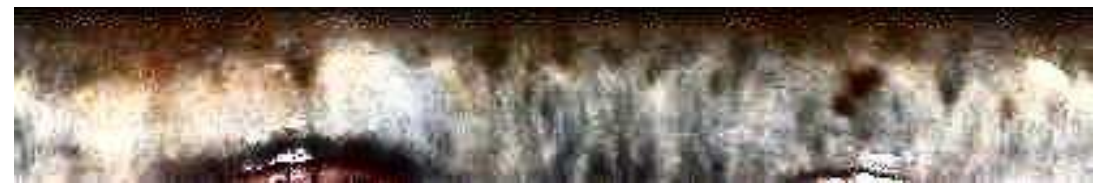

(b) CIE Lab enhanced sRGB image.

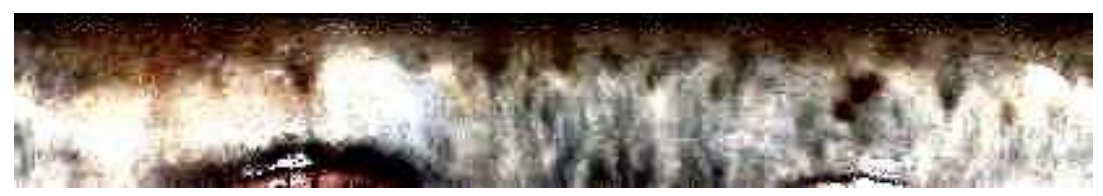

(c) YCbCr enhanced sRGB image.

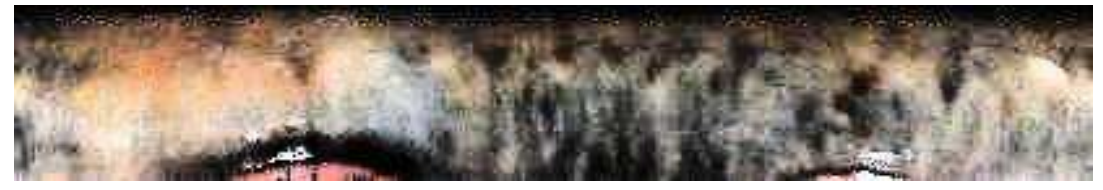

(d) HSV enhanced sRGB image.

Figure 3.1: Comparison of contrast enhancement techniques on a segmented iris image. 


\begin{tabular}{|l|c|c|c|c|c|c|}
\hline & $\begin{array}{c}\text { Average } \\
\text { Intensity }\end{array}$ & $\begin{array}{c}\text { Average } \\
\text { Contrast }\end{array}$ & Smoothness & $\begin{array}{c}\text { 3rd } \\
\text { Moment }\end{array}$ & Uniformity & Entropy \\
\hline RGB & 94.4404 & 31.3835 & 0.0165 & 0.1014 & 0.0330 & 5.1999 \\
\hline Lab Hist. Eq. & 133.8794 & 74.8890 & 0.0821 & 0.6006 & 0.0393 & 5.3187 \\
\hline YCbCr Hist. Eq. & 135.0211 & 81.9943 & 0.0965 & -0.1588 & 0.0554 & 5.0907 \\
\hline HSV Hist. Eq. & 110.5377 & 74.3239 & 0.0808 & 2.0070 & 0.0260 & 5.5605 \\
\hline
\end{tabular}

Table 3.1: Measurement index for contrast within the iris template. For all indices except uniformity a higher score represents greater amounts of texture.

ponents when compared to the original image, without preprocessing. No single enhancement method dominates all texture components of the measurement index but, instead, depending on characteristic of the single component of the measurement index, different enhancement techniques show to higher texture measurement. From this test it can not be determined which technique provides the best enhancement, but it is clear that these techniques should have a positive effect on matching performance (Figures 3.2, 3.3 and 3.4).

\begin{tabular}{|l|c|c|c|}
\hline Color Class & R & G & B \\
\hline Brown & $14.7 \%$ & $16.1 \%$ & $16.3 \%$ \\
\hline L. Brown/Green & $9.6 \%$ & $11.7 \%$ & $10.9 \%$ \\
\hline Blue & $7.8 \%$ & $8.1 \%$ & $8.3 \%$ \\
\hline Database & $9.6 \%$ & $11.8 \%$ & $10.9 \%$ \\
\hline
\end{tabular}

Table 3.2: The EER for the images enhanced via HSV histogram equalization.

\begin{tabular}{|l|c|c|c|}
\hline Color Class & R & G & B \\
\hline Brown & $16 \%$ & $17.8 \%$ & $17.7 \%$ \\
\hline L. Brown/Green & $11.2 \%$ & $11.3 \%$ & $12.2 \%$ \\
\hline Blue & $10.9 \%$ & $10.3 \%$ & $10.6 \%$ \\
\hline Database & $11.6 \%$ & $11.7 \%$ & $12.2 \%$ \\
\hline
\end{tabular}

Table 3.3: The EER for the images enhanced via CIE Lab histogram equalization. 


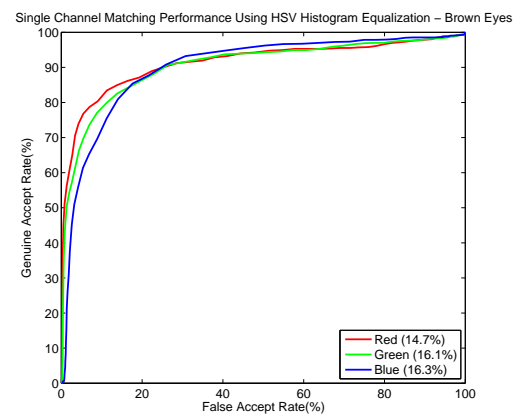

(a) Brown Eye Color Class

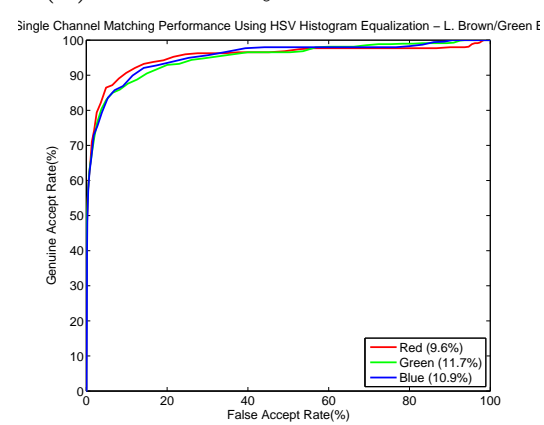

(b) Light Brown/Green Eye Color Class

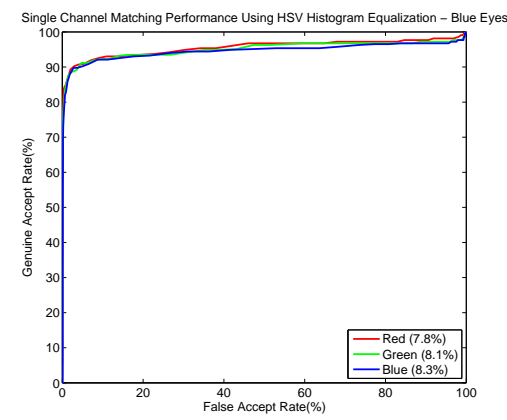

(c) Blue Eye Color Class

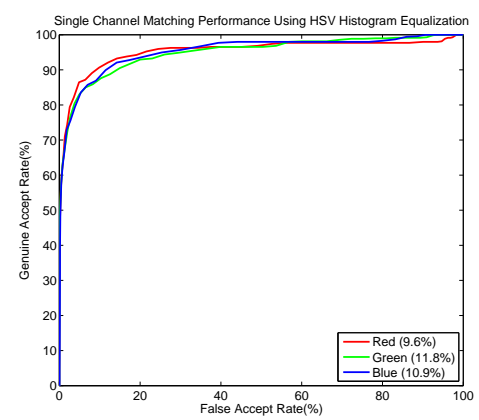

(d) Total Database and Fusion

Figure 3.2: Matching performance of images enhanced using HSV histogram equalization. 


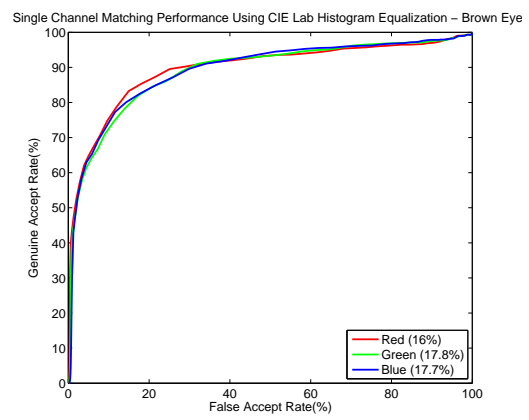

(a) Brown Eye Color Class

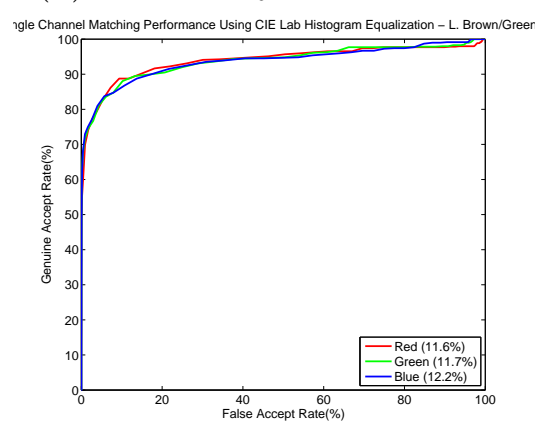

(b) Light Brown/Green Eye Color Class

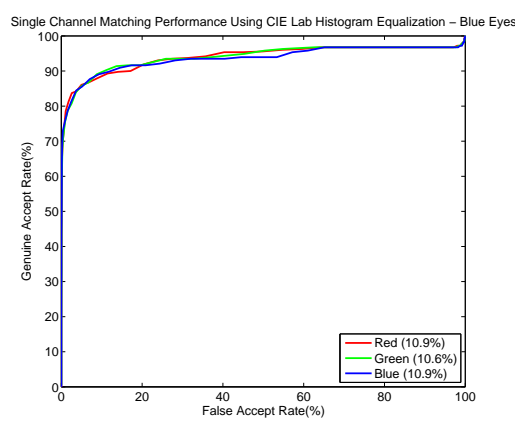

(c) Blue Eye Color Class

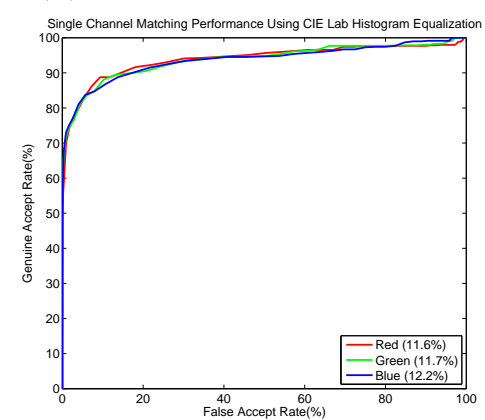

(d) Total Database and Fusion

Figure 3.3: Matching performance of images enhanced using CIE Lab histogram equalization. 


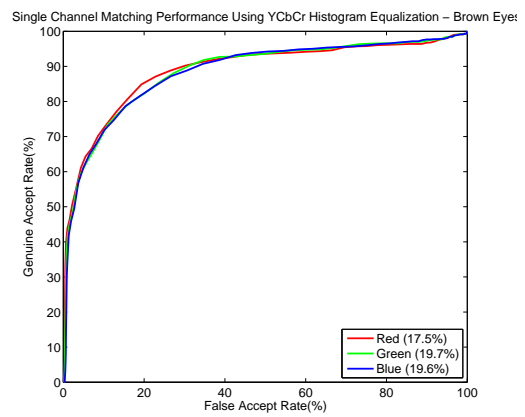

(a) Brown Eye Color Class

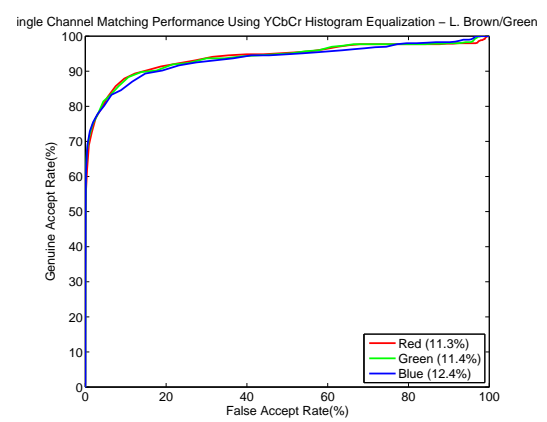

(b) Light Brown/Green Eye Color Class

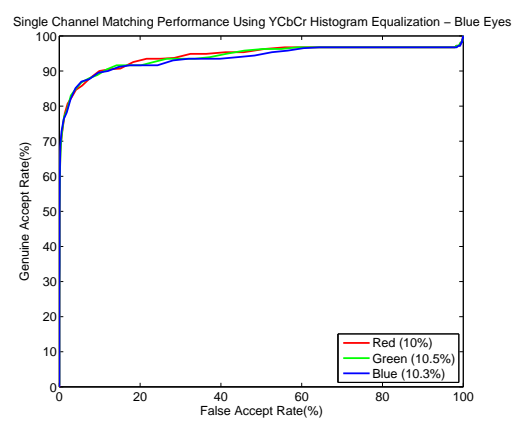

(c) Blue Eye Color Class

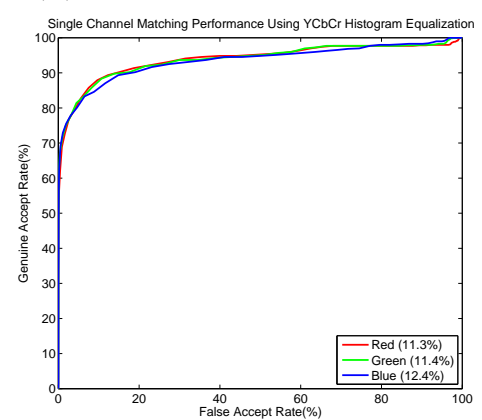

(d) Total Database and Fusion

Figure 3.4: Matching performance of images enhanced using YCbCr histogram equalization. 


\begin{tabular}{|l|c|c|c|}
\hline Color Class & R & G & B \\
\hline Brown & $17.5 \%$ & $19.7 \%$ & $19.6 \%$ \\
\hline L. Brown/Green & $11.3 \%$ & $11.4 \%$ & $12.4 \%$ \\
\hline Blue & $10 \%$ & $10.5 \%$ & $10.3 \%$ \\
\hline Database & $11.3 \%$ & $11.4 \%$ & $12.4 \%$ \\
\hline
\end{tabular}

Table 3.4: The EER for the images enhanced via YCbCr histogram equalization.

The matching performance reinforces the results shown in Table 3.1. Overall, there is an increase in performance when compared to the same database of non-enhanced sRGB images (Figure 3.5 and Table 3.5). The increase in performance varied depending on the eye color class, with eyes which typically contain more texture in the visible spectrum (blue) having the highest increase in performance.

\subsection{Iris Recognition in Alternative Color Spaces}

All iris images used in this work were captured in the sRGB color space and until now have been analyzed within this same color space. The intentions of this work is to determine if other color spaces will potentially better represent the texture of the iris and, therefore, provide better recognition performance. Four color space transforms are utilized: CIE Lab, YCbCR, HSV and CMYK. Once iris localization, unwrapping and normalization are complete the templates are transformed to one of the five previously mentioned color spaces. Within the new color space, encoding and matching are applied on a per channel basis, where images in each channel are explicitly matched against other images of the same channel (e.g. R-R, G-G, B-B). Matching performance is examined across all users as well as segregated based on eye color classes. The emphasis on performance in an individual color class, helps to demonstrate the increased role eye color has when imaging the iris in the visible spectrum.

Matching performance is judged both on the visual interpretation of the ROC curve and the EER. In all ROC curves, the EER is displayed within the legend. The overall performance 
is decreased since no image preprocessing or enhancement was utilized. This ensures that the only influence on matching performance is the color space transforms. It was concluded that preprocessing techniques would effect each color space differently and, if applied in the sRGB domain prior to the color space transform, it could potentially give an unfair advantage to matching performance in the sRGB domain since it is difficult to predict its impact once the images are transformed. Performance may also suffer due to the difficult nature of the UBIRIS database. Since the database is designed to include non-ideal images, much of the data contains iris occlusion from the eyelids and/or the image may not be in focus [52,53].

\begin{tabular}{|l|c|c|c|}
\hline Color Class & R & G & B \\
\hline Brown & $13.7 \%$ & $16.1 \%$ & $22.4 \%$ \\
\hline L. Brown/Green & $12.1 \%$ & $12.8 \%$ & $12.2 \%$ \\
\hline Blue & $12.4 \%$ & $12.6 \%$ & $11.7 \%$ \\
\hline Database & $12.2 \%$ & $12.8 \%$ & $12.3 \%$ \\
\hline
\end{tabular}

Table 3.5: The EER for the sRGB color space.

\begin{tabular}{|l|c|c|c|}
\hline Color Class & H & S & S \\
\hline Brown & $44.6 \%$ & $21.9 \%$ & $19.6 \%$ \\
\hline L. Brown/Green & $37 \%$ & $9 \%$ & $13.2 \%$ \\
\hline Blue & $29 \%$ & $9.9 \%$ & $13.1 \%$ \\
\hline Database & $36.9 \%$ & $8.4 \%$ & $16 \%$ \\
\hline
\end{tabular}

Table 3.6: The EER for the HSV color space.

When analyzing matching performance in the visible domain, it is essential that not only high performance is witnessed, but also that performance remain consistently robust across all eye color classes. In other words, if there is heavy fluctuation in performance between eye color classes, then the performance within the respective color class cannot be considered high. 


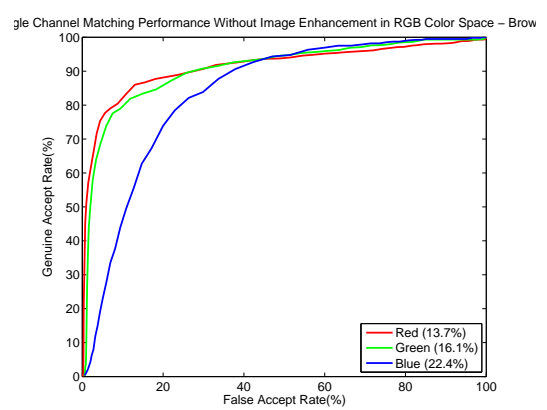

(a) Brown Eye Color Class

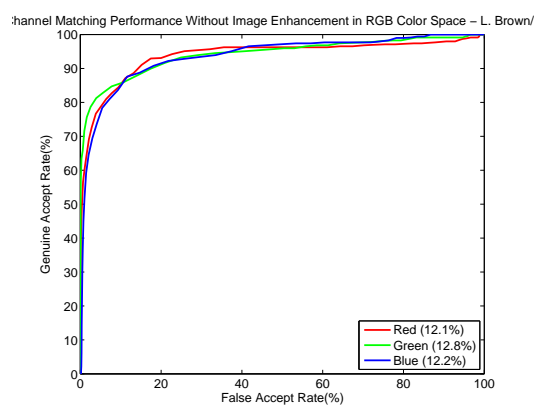

(b) Light Brown/Green Eye Color Class

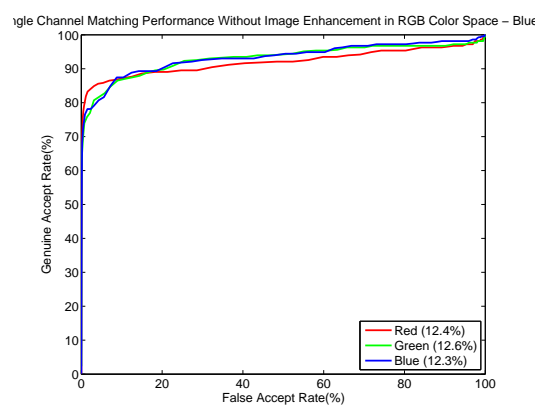

(c) Blue Eye Color Class

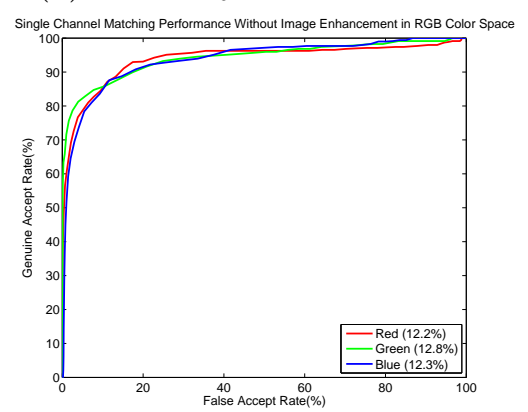

(d) Total sRGB Database and Fusion

Figure 3.5: Matching performance in the sRGB color space. 


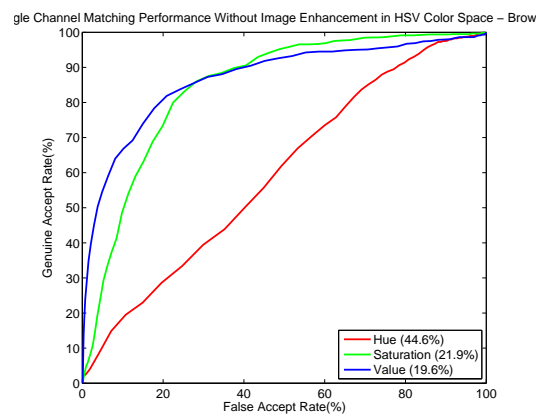

(a) Brown Eye Color Class

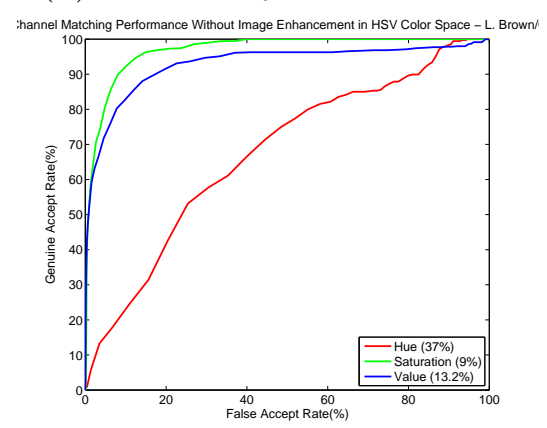

(b) Light Brown/Green Eye Color Class

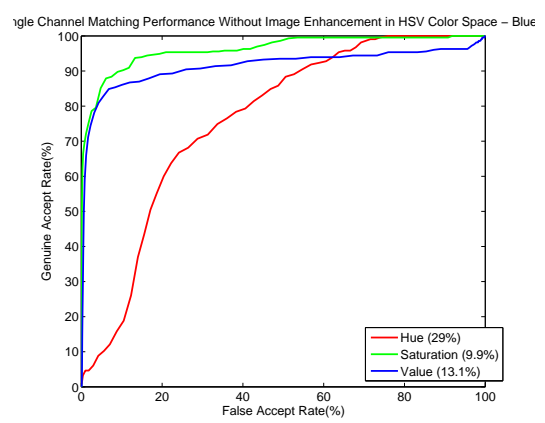

(c) Blue Eye Color Class

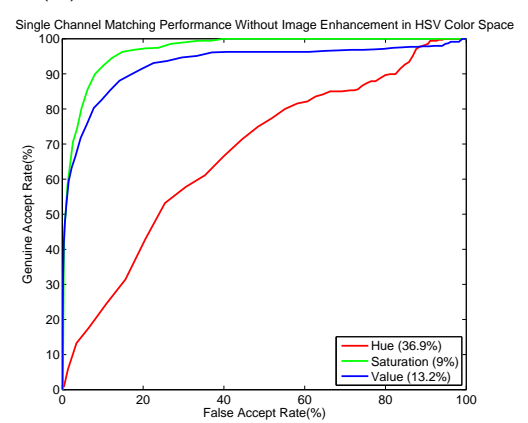

(d) Total HSV Database and Fusion

Figure 3.6: Matching performance in the HSV color space. 


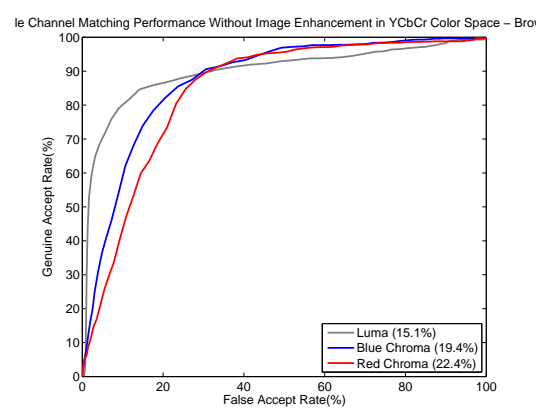

(a) Brown Eye Color Class

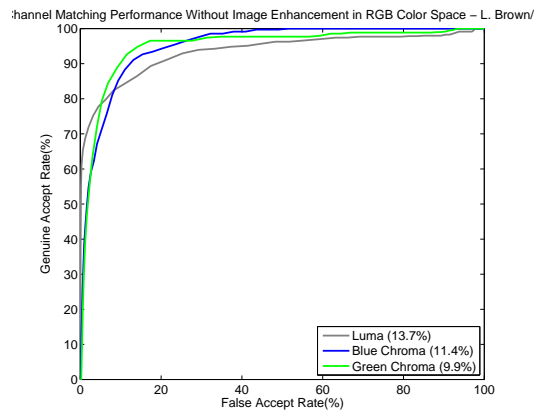

(b) Light Brown/Green Eye Color Class

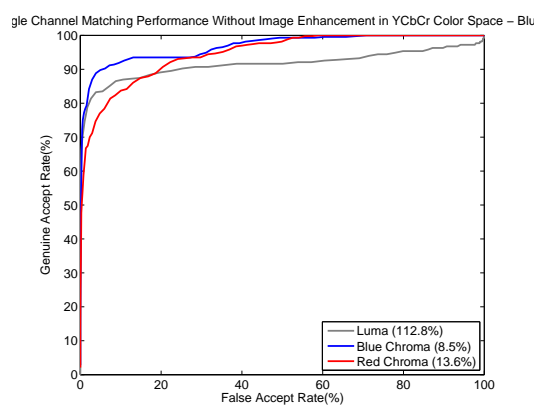

(c) Blue Eye Color Class

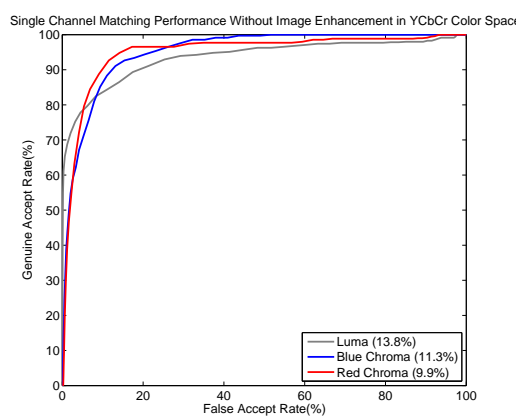

(d) Total YCbCr Database and Fusion

Figure 3.7: Matching performance in the $\mathrm{YCbCr}$ color space. 


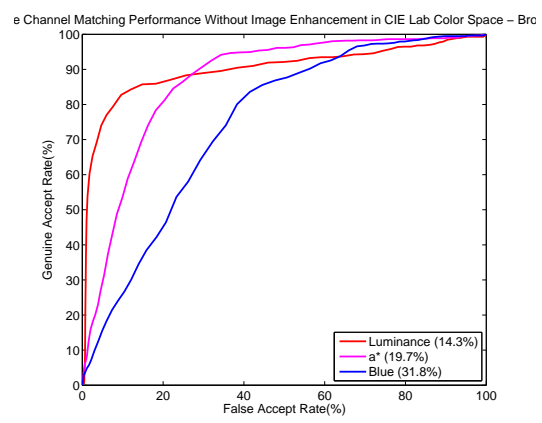

(a) Brown Eye Color Class

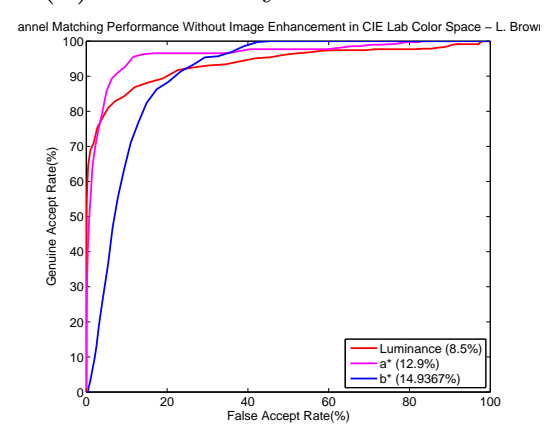

(b) Light Brown/Green Eye Color Class

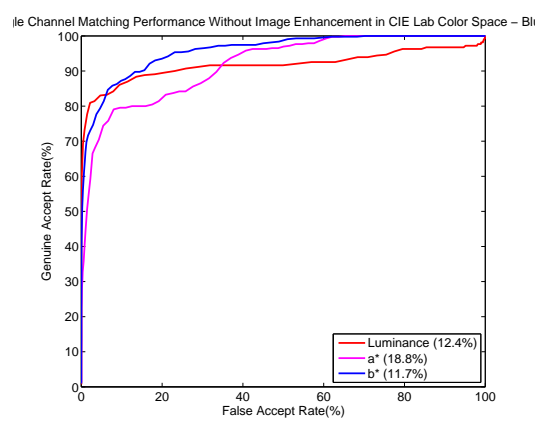

(c) Blue Eye Color Class

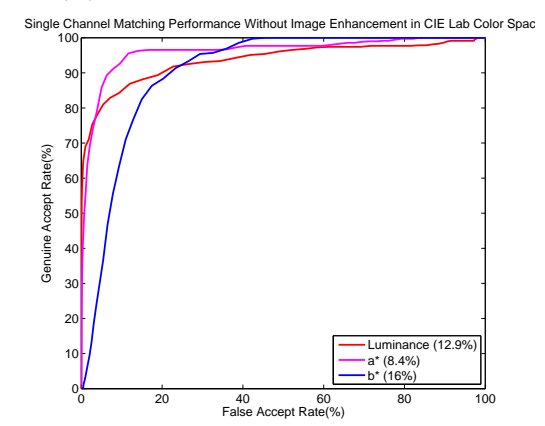

(d) Total CIE Lab Database and Fusion

Figure 3.8: Matching performance in the CIE Lab color space. 


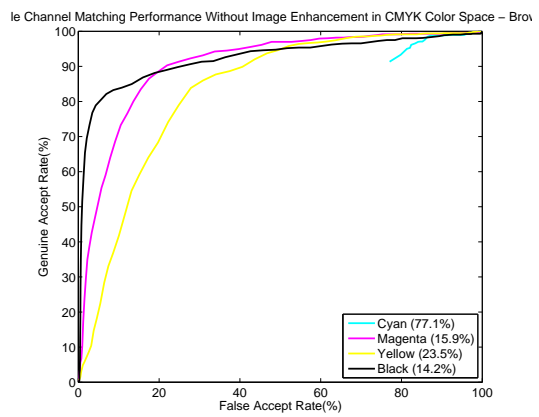

(a) Brown Eye Color Class

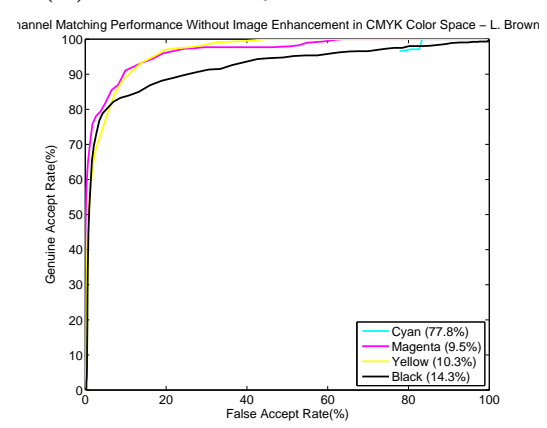

(b) Light Brown/Green Eye Color Class

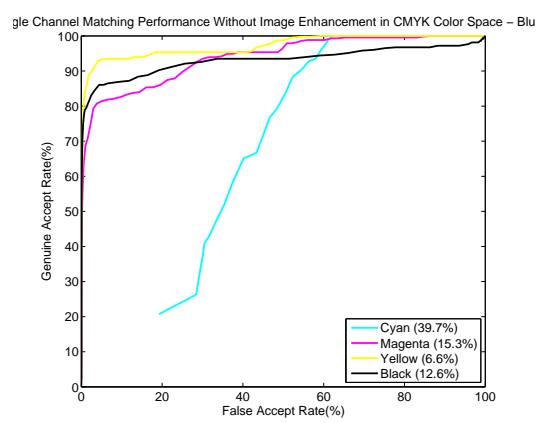

(c) Blue Eye Color Class

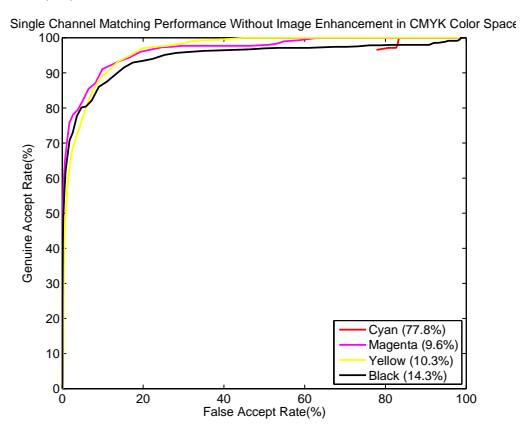

(d) Total CMYK Database and Fusion

Figure 3.9: Matching performance in the CMYK color space. 


\begin{tabular}{|l|c|c|c|}
\hline Color Class & Y & Cb & Cr \\
\hline Brown & $15.1 \%$ & $19.4 \%$ & $22.4 \%$ \\
\hline L. Brown/Green & $13.7 \%$ & $11.4 \%$ & $9.9 \%$ \\
\hline Blue & $12.8 \%$ & $8.5 \%$ & $13.6 \%$ \\
\hline Database & $13.8 \%$ & $11.3 \%$ & $9.9 \%$ \\
\hline
\end{tabular}

Table 3.7: The EER for the YCbCr color space.

\begin{tabular}{|l|c|c|c|}
\hline Color Class & L & a & b \\
\hline Brown & $14.3 \%$ & $19.7 \%$ & $31.8 \%$ \\
\hline L. Brown/Green & $8.5 \%$ & $12.9 \%$ & $16 \%$ \\
\hline Blue & $12.4 \%$ & $18.8 \%$ & $11.7 \%$ \\
\hline Database & $12.9 \%$ & $8.4 \%$ & $16 \%$ \\
\hline
\end{tabular}

Table 3.8: The EER for the CIE Lab color space.

\begin{tabular}{|l|c|c|c|c|}
\hline Color Class & $\mathbf{C}$ & $\mathbf{M}$ & $\mathbf{Y}$ & $\mathbf{K}$ \\
\hline Brown & $77.1 \%$ & $15.9 \%$ & $23.5 \%$ & $14.2 \%$ \\
\hline L. Brown/Green & $77.8 \%$ & $9.5 \%$ & $10.3 \%$ & $14.3 \%$ \\
\hline Blue & $39.7 \%$ & $15.3 \%$ & $6.6 \%$ & $12.6 \%$ \\
\hline Database & $77.8 \%$ & $9.6 \%$ & $10.3 \%$ & $14.3 \%$ \\
\hline
\end{tabular}

Table 3.9: The EER for the CMYK color space. 


\subsubsection{Cross-Band Correlation}

An analysis of cross-band correlation of matching scores is provided in Tables 3.10, 3.11, 3.12, 3.13 and 3.14. Band correlation is derived across each eye color class for both the genuine and imposter matching scores.

\begin{tabular}{|l|c|c|c|c|c|c|c|c|c|}
\hline & \multicolumn{3}{|c|}{ Brown Eye } & \multicolumn{3}{c|}{ Light Brown/Green Eye } & \multicolumn{3}{c|}{ Blue Eye } \\
\hline & R-G & R-B & G-B & R-G & R-G & R-G & R-G & R-B & G-B \\
\hline Genuine & .7558 & .2591 & .5533 & .872 & .6542 & .8561 & .9168 & .9097 & .9732 \\
\hline Imposter & .6192 & .3556 & .7569 & .6238 & .3655 & .7752 & .5664 & .4192 & .8668 \\
\hline
\end{tabular}

Table 3.10: Matching score cross-band correlation in the sRGB color space.

\begin{tabular}{|l|c|c|c|c|c|c|c|c|c|}
\hline & \multicolumn{3}{|c|}{ Brown Eye } & \multicolumn{4}{c|}{ Light Brown/Green Eye } & \multicolumn{3}{c|}{ Blue Eye } \\
\hline & H-S & H-V & S-V & H-S & H-V & S-V & H-S & H-V & S-V \\
\hline Genuine & .7171 & .3511 & .3251 & -.0501 & -.1467 & .3823 & .301 & .2761 & .7216 \\
\hline Imposter & .6071 & .2983 & .3806 & .5009 & .1282 & .2552 & .4677 & -.0092 & .2141 \\
\hline
\end{tabular}

Table 3.11: Matching score cross-band correlation in the HSV color space.

\begin{tabular}{|l|c|c|c|c|c|c|c|c|c|}
\hline & \multicolumn{3}{|c|}{ Brown Eye } & \multicolumn{3}{c|}{ Light Brown/Green Eye } & \multicolumn{3}{c|}{ Blue Eye } \\
\hline & Y-Cb & Y-Cr & Cb-Cr & Y-Cb & Y-Cr & Cb-Cr & Y-Cb & Y-Cr & Cb-Cr \\
\hline Genuine & .1883 & .2513 & .7319 & .4951 & .5374 & .5407 & .6688 & .5908 & .8226 \\
\hline Imposter & .3896 & .3208 & .7965 & .3238 & .3406 & .8368 & .3584 & .4012 & .9014 \\
\hline
\end{tabular}

Table 3.12: Matching score cross-band correlation in the $\mathrm{YCbCr}$ color space.

These results indicate there is a strong relationship between the two chromaticity components of the CIE Lab and YCbCr color spaces, respectfully. High correlation relationships in other color spaces such as the G-B bands of blue colored sRGB images, also indicate a close relationship in the nature of the textural data present. 


\begin{tabular}{|l|c|c|c|c|c|c|c|c|c|}
\hline & \multicolumn{3}{|c|}{ Brown Eye } & \multicolumn{3}{c|}{ Light Brown/Green Eye } & \multicolumn{3}{c|}{ Blue Eye } \\
\hline & L-a & L-b & a-b & L-a & L-b & a-b & L-a & L-b & a-b \\
\hline Genuine & .2437 & .0781 & .7516 & .6438 & .313 & .2818 & .5103 & .5908 & .7721 \\
\hline Imposter & .3254 & .4066 & .7749 & .3139 & .3167 & .6116 & .4036 & .3975 & .8156 \\
\hline
\end{tabular}

Table 3.13: Matching score cross-band correlation in the CIE Lab color space.

\begin{tabular}{|l|c|c|c|c|c|c|}
\hline & \multicolumn{7}{|c|}{ Brown Eye } \\
\hline & C-M & C-Y & M-Y & C-K & M-K & Y-K \\
\hline Genuine & .6336 & .6101 & .8878 & .2037 & .3304 & .2055 \\
\hline Imposter & .4927 & .6641 & .8514 & .2634 & .3834 & .4203 \\
\hline & \multicolumn{7}{|c|}{ Light Brown/Green Eye } \\
\hline & C-M & C-Y & M-Y & C-K & M-K & Y-K \\
\hline Genuine & -.0735 & -.2109 & .7244 & .0023 & .5791 & .4013 \\
\hline Imposter & .2939 & .3901 & .8349 & .1279 & .2271 & .2912 \\
\hline & \multicolumn{7}{|c|}{ Blue Eye } \\
\hline & C-M & C-Y & M-Y & C-K & M-K & Y-K \\
\hline Genuine & .187 & -.1658 & .7278 & .0877 & .6998 & .7856 \\
\hline Imposter & .4119 & .4513 & .8798 & -.0617 & .2309 & .2395 \\
\hline
\end{tabular}

Table 3.14: Matching score cross-band correlation in the CMYK color space. 


\subsection{2 sRGB Cross-Band Matching}

Analysis of the correlation between color band matching performance has shown that in the sRGB color space matching, performance is affected by eye color. To further substantiate these findings, cross-band matching was performed in the sRGB color space. Single-band matching using Gabor based texture encoding was implemented across all three bands. The matching performance of red versus green, red versus blue and green versus blue were observed (Figure 3.10 and Table 3.15).

\begin{tabular}{|l|c|c|c|c|}
\hline Color Class & Brown Eyes & L. Brown/Green Eyes & Blue Eyes & Database \\
\hline R-G & $29 \%$ & $43 \%$ & $47.4 \%$ & $36.7 \%$ \\
\hline R-B & $38.7 \%$ & $52 \%$ & $69.8 \%$ & $48.4 \%$ \\
\hline G-B & $29.2 \%$ & $31.8 \%$ & $29.6 \%$ & $29.8 \%$ \\
\hline
\end{tabular}

Table 3.15: The EER for cross-band matching of sRGB image bands.

Based on the previous finding of this work, it was expected that performance of lighter colored eyes would out perform that of the darker brown eyes due to the optical properties of the iris in the visible domain. Since a high contrast and better quality iris image is present in lighter color eyes it was expected that matching performance would be better for lighter colored eyes. The performance results show the contrary to be true, eyes of the dark brown color class performance better in cross-band matching.

\subsection{Discussion and Summary}

Multiple color image enhancement techniques were introduced in this chapter with the intent of enhancing the contrast of the image without altering the chromaticity. These algorithms were tested and compared, each showing an increase in performance versus the original non-enhanced image. No one single enhancement algorithm dominates performance across all possible combination of color classes and color bands, but instead the performance of all algorithms is comparable. 


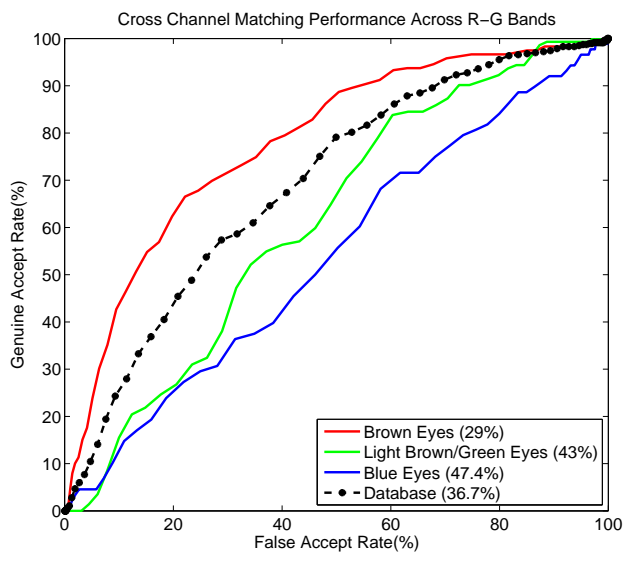

(a) Matching Performance across R-G Bands

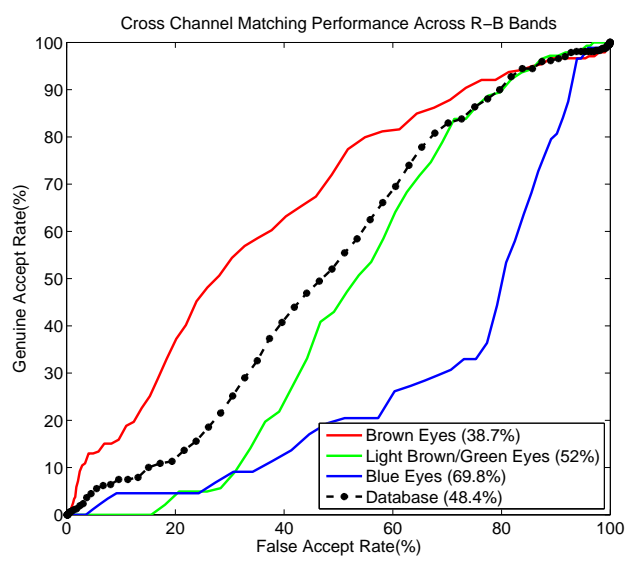

(b) Matching Performance across R-B Bands

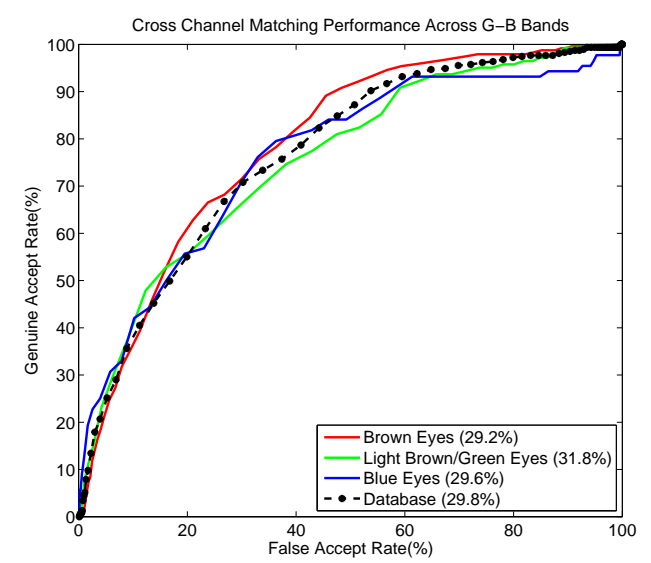

(c) Matching Performance across G-B Bands

Figure 3.10: Matching performance of cross-band comparison of sRGB image bands. 
It should be noted that the chromaticity does not remain constant after image enhancement and each algorithm perturbs the chromaticity in a certain way. Since the objective of each technique is to increase matching performance, slight disturbances in chromaticity are acceptable and are ultimately dependant on the enhancement algorithm. Therefore, the chromaticity of all images will be affected in the same manner nullifying any negative affects that may have resulted from applying the algorithm.

Iris recognition in alternative color spaces was also examined in this chapter. No single color space transformation was found to increase matching performance across all components, but instead individual components of certain color spaces showed potential as ideal candidates for iris recognition. In color spaces such as $\mathrm{CIE}$ Lab and $\mathrm{YCbCr}$, the luminosity functions showed consistently high performance across all eye color classes. When the chromaticity is completely segmented from the luminosity, such as in the CIE Lab and YCbCr color spaces, the chromaticity shows to have high correlations between its two components indicating that their performance and viability as templates are similar. Overall, the traditional color space, sRGB, showed strong performance, but the per channel performance can be heavily dependant on the eye color class. Cross-band correlation in the sRGB also varied with eye color class, again showing that matching performance in the sRGB color space varies based on the eye color class. Like in the sRGB color space, the CMYK performance was significantly dependent on the eye color class. Exceptionally poor performance is seen in the band representing cyan, especially for brown and light brown/green eyes. This is understandable considering the spectral reflectance of cyan contains zero red, which corresponds to a majority of the chromaticity in a dark brown eye. Performance in the HSV color space mirrored those of the YCbCr and CIE Lab. The value, which represents the brightness of the image, contained a majority of the texture resulting in more robust texture and better matching performance. Unlike the luminosity functions of the YCbCr and CIE Lab color spaces, the value of the image is dependant on the brightness of color in the image and more susceptible to variations in eye color. The hue and saturation components are weakened by templates which have little variation in color across the iris. Dark brown eyes, 
for example, show little to no contrast when only a single, rich pigment is present in both the anterior and posterior layers of the iris.

For optimal matching performance throughout a database which consists of users of all eye colors a combination of components across multiple color spaces may induce the best performance. Luminosity functions, such as the $\mathbf{L}$ component of CIE Lab, continued to show high performance across all eye color classes. This was also noticed in the chromaticity components of the $\mathrm{YCbCr}$ color space. Therefore, by extracting components of various color spaces and then fusing the results at the score level, optimal performance with texture-based encoding of color images may be achieved.

Finally, cross-band matching was performed across the entire sRGB database. The results were contrary to what was to be expected based on the previous work discussed in this chapter. Across all possible image band matching combinations, brown eyes out performed all other color classes. This is very surprising considering the loss of image quality of dark brown eyes as the image band wavelength decreases. Because of this loss in quality, it would be expected that the template created in the red band would not mirror that of the green band as closely as the red and green band templates of a light blue eye would. At the time of publication the explanation of why this phenomena exists is unknown, but may likely be due to the small database size and the preponderance of brown eyes in the data. 


\section{Chapter 4}

\section{Summary and Future Work}

\subsection{Summary of Work}

Research in multispectral and color iris recognition is much less mature than those involving near-IR. Due to this, the availability of robust, high quality multispectral data is limited to only a few choice databases. The only multispectral (i.e., color and near-IR images) iris database available to the author at the time of publication is the WVU Multispectral Iris database that was used throughout this work. As previously stated, this database is in it's early stages of development and does not meet the goals of size or robustness. It has been the author's intention to develop this database in both size and diversity of eye color, but due to limitations in time, availability of data and institutional restrictions, this was not possible. Due to these factors, this thesis focuses on the techniques that can be used for multispectral and color iris recognition rather, than attempting to quantify the results using larger datasets.

In this thesis, techniques to improve recognition performance using multispectral and color iris images was discussed. First, a dynamic score level fusion algorithm was implemented. The algorithm utilizes the additional bands of the multispectral image to increase performance at the score level. Fusion decisions are made based on the textural information available within each band of the image. The ideal bands for fusion tend to be dependent on the eye color of the 
user, where darker eyes favor the near-IR and red bands, while lighter eyes favor the green and blue bands. The fusion algorithm was utilized by two different texture encoding schemes: Gabor based and LBP based methods. The increase in performance due to fusion was not as significant for templates encoded by Gabor filters compared to those encoded using LBP. Since the initial recognition performance using Gabor based encoding was much greater than that of the LBP based approach, the potential for an increase in recognition performance due to fusion was not as strong. Therefore the overall increase in performance resulting from fusion was not as apparent for the Gabor based approach as it was for the LBP. Also, the dynamic score level fusion algorithm was applied to an image database composed completely of sRGB images. Without the presence of the near-IR band, the fusion performance was greatly impacted, in some cases showing to be worse then single-band matching performance. This further emphasizes the strength of the near-IR band in iris recognition. But, since the fusion algorithm still required that a sum of two scores take place, darker eye color users were required to fuse sub-par scores which led to poor fusion performance. This resulting in a decline in performance when compared to single-band matching performance, thereby leading to the final conclusion that within the visible spectrum, texture-based encoding is not robust to changes in eye color and, in some cases, irides with dark eye color and low contrast, perform particularly poor due to the inability of illumination in the visible spectrum to penetrate the iris.

The addition of color channels to iris recognition presents the opportunity to investigate various color space transforms. Performance within the CIE Lab, YCbCr, CMYK and HSV color spaces were compared with the default matching performance in the sRGB color space with the intention to determine if an optimal color space for iris recognition exists. The UBIRIS database, which is comprised solely of sRGB images, was utilized for this experiment and a detailed evaluation was completed. Results reiterate much of what was already witnessed through our work with texture-based encoding and fusion in the visible domain. Color spaces, such as YCbCr and CIE Lab, which isolate the luminosity component from chromaticity, demonstrated that if the color component of the image was removed then the relationship between eye color 
and matching performance became less apparent, thus resulting in more uniform performance across all eye colors. On the contrary if the chromaticity of the image is isolated, such as in the hue component of the HSV color space, performance once again became correlated with the eye color class: dark brown eyes fared poorly due to their uniform, low textured color while lighter irides, which are more likely to be multichromatic, show better performance. Multichromatic eyes, where the melanin content varies between the posterior and anterior layers of the eye, showed more texture and, thus, were better candidates for texture-based encoding. The impact of eye color can be further seen through cross-band correlation where strong relationships exist between the chromaticity components of each of the CIE Lab and YCbCr color spaces. In both color spaces, the correlation between luminosity and each component of chromaticity is lower, substantiating the claim that the texture present within the luminosity components of the image will remain constant across all eye colors while the texture residing within the chromaticity components does not. From this it can be concluded that no single color space provides an ideal medium for iris recognition. Instead, ideal components of the color image, such as the luminosity, so to be robust across all eye colors and may be beneficial to iris recognition.

Finally, multiple color image enhancement techniques (CIE Lab histogram equalization, YCbCr histogram equalization and HSV histogram equalization) were examined. Each algorithm tested was designed with the intention to increase the overall contrast of the iris texture with the least impact on the chromaticity. To achieve this, the images were transferred to a color space where the luminosity component could be isolated from the chromaticity. Here, the luminosity could be varied through the use of histogram equalization without altering the chromaticity of the image. The images were then transformed back to their native sRGB color space. Each of the three techniques showed an increase in bothperceived textural content and matching performance compared to the original image. An increase of approximately $2-3 \%$ in EER was witnessed with all preprocessing techniques. Ideally, the enhancement technique would have no effect on the chromaticity of the image, but this was not found to be the case. Since the change in chromaticity is a direct result of an intentional operation that is applied to all images within 
a dataset, we speculate that the variation in chromaticity will be inconsequential at the end.

\subsection{Future Work}

Due to the lack of readily available commercial multispectral and color iris imaging devices it is difficult to access large, readily available multispectral and color iris databases. To further fortify the finding of this work, larger multispectral databases should be collected. An emphasis must be placed on collecting equal amounts of data across all eye color classes. Since the current multispectral database heavily favors dark brown and light brown/green eye colored eyes, as the collection continues, a conscience effort to collect more users with blue colored eyes would be advised until the database is equally distributed. A large, robust database may help substantiate the results found in this, as well as any previous and future work in multispectral iris recognition. To ensure that the database is diverse and statistically significant, for future work the author suggests that the database contain at least one-hundred users of each eye color class.

To fully understand the potential of iris recognition, larger portions of the electromagnetic spectrum should be explored. As this research works with images captured at wavelengths below that of a traditional commercial iris system, much of the near-IR spectrum is yet to be explored. Through the use of a hyperspectral imaging with device such as Xenics XEVA XS ${ }^{1}$ the iris can potentially be imaged up to wavelengths of $1.7 \mu m$, encompassing the entire nearIR spectrum. When imaging in the upper portions of the near-IR spectrum iris segmentation becomes nontrivial. Aqueous solution secreted from the eye provides a unique problem due to high absorption of energy in the extended near-IR causing the imaging of the sclera to become difficult [54]. Prior to imaging the iris in the extended near-IR, it must first be established that the eye can be safely illuminated within the imaging range. At the time of this work no previous publications in extended near-IR imaging or eye safety was available.

When the dynamic score level fusion rule was applied to the color iris database, the perfor-

\footnotetext{
${ }^{1}$ http://www.lot-oriel.com/site/pages_de_en/home/home/home.php
} 
mance drastically declined. Since the fusion algorithm requires two channels of the image to be fused, in some cases exceptionally poor data was included in the fusion process thus causing the poor performance. A metric must be introduced to judge the contrast scores of each band of the multispectral image and a threshold must be set where fusion is avoided if multiple templates are not of satisfactory quality. In some cases, such as for dark brown eyes, performance is so poor across the green and blue bands that overall matching performance suffers from fusion. Also, due to limitations in time the author did not have the opportunity to test the score level fusion algorithm with encoding schemes that are not texture-based. As discussed above, texture-based encoding schemes applied to color images perform poorly when compared to similar techniques applied in the near-IR band. By utilizing a more robust color iris encoding scheme, overall performance would not have been as dependant on eye color thereby removing the bias on performance as a result of a preponderance of brown irides. By having more robust single-band matching performance, the potential of the dynamic score level fusion algorithm may be better revealed.

The findings of this work showed that the matching performance associated with texturebased encoding varies with eye color and the specific band of the iris template. As perviously mentioned, work by Boyce et al. has shown that encoding schemes based on image intensity instead of texture are more robust to variations in user eye color. The author speculates that encoding schemes that include a measure of the chromaticity gradient calculated radially from the pupil to limbic boundary, as well as a quantization of the overall color of the image, may potentially lead to more robust matching performance across all eye color classes 


\section{References}

[1] A. K. Jain, "Biometric recognition: how do i know who you are?" in Proceedings of the IEEE 12th Signal Processing and Communications Applications (SPCA) Conference, 2004, pp. 3-5.

[2] A. K. Jain, A. Ross, and S. Pankanti, "Biometrics: A tool for information security," IEEE Transactions on Information Forensics and Security, vol. 1, no. 2, 2006.

[3] A. Jain, R. Bolle, and S. Pankanti, "Introduction to biometrics," in Biometrics: Personal Identification in a Networked Society, A. Jain, R. Bolle, and S. Pankanti, Eds. Kluwer Academic Publishers, 1999.

[4] A. K. Jain, A. Ross, and S. Prabhakar, "An introduction to biometric recognition," IEEE Transactions on Circuits and Systems for Video Technology, vol. 14, no. 1, pp. 4-20, 2004.

[5] S. G. Kong, J. Heo, B. R. Adibi, J. Paik, and M. A. Abidi, "Recent advances in visual and infrared face recognition - a review," Computer Vision and Image Understanding, vol. 97, no. 1, 2005.

[6] L. Torres, J.Y.Reutter, and L.Lorente, "The importance of the color information in face recognition," in Procceedings of International Conference on Image Processing (ICIP), Kobe, Japan, 1999, pp. 627-631.

[7] J. Wilder, P. J. Phillips, C. Jiang, and S. Wiener, "Comparison of visible and infra-red imagery for face recognition," in Proceedings of 2nd International Conference on Automatic Face and Gesture Recognition, Killington, VT, USA, 1996, pp. 182-187.

[8] J. Campbell, "Speaker recognition," in Biometrics: Personal Identification in a Networked Society, A. Jain, R. Bolle, and S. Pankanti, Eds. Kluwer Academic Publishers, 1999.

[9] L. O'Gorman, "Fingerprint verification," in Biometrics: Personal Identification in a Networked Society, A. Jain, R. Bolle, and S. Pankanti, Eds. Kluwer Academic Publishers, 1999. 
[10] J. Daugman, "Recognizing persons by their iris patterns," in Biometrics: Personal Identification in a Networked Society, A. Jain, R. Bolle, and S. Pankanti, Eds. Kluwer Academic Publishers, 1999.

[11] Y. Zhu, T. Tan, and Y. Wang, "Biometric personal identification based on iris pattern," in Proceedings of 15th International Conference of Pattern Recognition (ICPR), 2002, pp. 2801-2804.

[12] L. Ma, Y. Wang, and T. Tan, "Iris recognition based on multichannel gabor filtering," in Proceedings of 5th Asian Conference of Computer Vision (ACCV), Melbourne, Australia, 2002, pp. 1-5.

[13] L. Flom and A. Safir, "Iris recognition system," U.S. Patent 4641 349, February 03, 1987.

[14] R. L. Davis and P. D. Becherer, "Techniques for improved soft lens fitting," Contact Lens Spectrum, no. 117, 2005.

[15] S. M. Carden, R. E. Boissy, P. J. Schoettker, and W. V. Good, "Albinism: Modern molecular diagnosis," British Journal of Opthamology, vol. 82, no. 2, pp. 189-195, 1998.

[16] A. R. Wielgus and T. Sarna, "Melanin in human irides of different color and age of donors," Pigment Cell Research, vol. 18, no. 6, pp. 454-464, 2005.

[17] J. C. Khan, H. Shahid, D. A. Thurlby, M. Bradley, D. G. Clayton, A. T. Moore, A. C. Bird, and J. R. W. Yates, "Age related macular degeneration and sun exposure, iris colour, and skin sensitivity to sunlight," British Journal of Ophthalmology, vol. 90, pp. 29-32, 2006.

[18] C. Boyce, "Multispectral iris recognition analysis: Techniques and evaluation," MSEE Dissertation, West Virginia University, 2006.

[19] National Science and Technology Council (NSTC) Subcomitee on Biometrics, "Iris recognition," August 2006. [Online]. Available: http://www.biometrics.gov/Docs/irisrec. pdf

[20] A. Noore, "Highly robust biometric smart card design," in IEEE Transactions on Consumer Electronics, vol. 46, no. 4, 2000, pp. 1059-1063.

[21] A. A. Ross, K. Nandakumar, and A. K. Jain, Handbook of Multibiometrics. Springer Science+Business Media, LLC, 2006.

[22] A. Ross, A. K. Jain, and J.-Z. Qian, "Information fusion in biometrics," Lecture Notes in Computer Science (A longer version appears in Pattern Recognition Letters, Vol. 24, Issue 13, pp. 2115-2125, September, 2003., vol. 2091, pp. 354-359, 2001. [Online]. Available: citeseer.ist.psu.edu/ross03information.html 
[23] J. Hewett, V. Nadeau, J. Ferguson, H. Moseley, S. Ibbotson, J. W. Allen, W. Sibbett, and M. Padgett, "The application of a compact multispectral imaging system with integrated excitation source to in vivo monitoring of fluorescence during topical photodynamic therapy of superficial skin cancers," Photochemistry and Photobiology, vol. 73, no. 3, pp. 278-282, 2001.

[24] O. Hellwich and C. Wiedemann, "Multisensor data fusion for automated scene interpretation." [Online]. Available: citeseer.ist.psu.edu/416796.html

[25] B. Abidi, S. Huq, and M. Abidi, "Fusion of visual, thermal, and range as a solution to illumination and pose restrictions in face recognition." in IEEE Carnahan Conf. Security Technology, Albuquerque, NM, USA, October 2004, pp. 325-330.

[26] R. Rowe, K. Nixon, and S. Corcoran, "Multispectral fingerprint biometrics," in Proceedings of the IEEE Workshop on Information Assurance and Security, West Point, NY, USA, 2005.

[27] C. Poynton, Digital Video and HDTV Algorithms and Interfaces. New York, New York, USA: Morgan Kaufmann, 2003.

[28] E. Wolf, Progress in Optics. Ann Arbor, Michigan, USA: North-Holland Publishing Company, 1961, p. 213.

[29] D. Malacara, Color Vision and Colorimetry: Theory and Applications. SPIE Publications, 2002.

[30] L. Gartner and J. Hiatt, Color Atlas of Histology. Baltimore, MD, USA: Lippincott, Williams and Wilkins, 2006, pp. 217-234.

[31] R. C. Maclaurin, Light. The Columbia University Press, 1909.

[32] C. Poynton, "YUV and luminance considered harmful: A plea for precise terminology in video," www.inforamp.net, 2001.

[33] Adobe Systems Incorporated, PDF Reference Fifth Edition: Adobe Portable Document Format Version 1.6. Adobe Press, 2004.

[34] S. Lee, K. K. Park, and J. Kim, "Robust fake iris detection based on variation of the reflectance ration between the iris and sclera," in Proceedings of Biometrics Consortium Conference, Baltimore, Maryland, USA, 2006, pp. 9.19-9.21.

[35] A. Vogel, C. Dlugos, R. Nuffer, and R. Birngruber, "Optical properties of human sclera, and their consequences for transscleral laser applications," in Lasers in Surgery and Medicine, vol. 11, no. 4, 1991, pp. 331-340. 
REFERENCES

[36] E. V. Koblova, A. N. Bashkatov, E. A. Genina, V. V.Tuchin, and V. V. Bakutkin, "Estimation of melanin content in iris of human eye," in Proceedings of Society for Photo-Optical Instrumentation Engineers (SPIE), vol. 5688, 2005, pp. 302-311.

[37] C. Boyce, A. Ross, M. Monaco, L. Hornak, and X. Li, "Multispectral iris analysis: A preliminary study," in Proceedings of IEEE Computer Society Workshop on Biometrics, June 2006.

[38] J. G. Daugman, "High confidence visual recognition of persons by a test of statistical independence," IEEE Transactions on Pattern Analysis and Machine Intelligence (PAMI), vol. 15, no. 11, pp. 1148-1161, 1993.

[39] J. Daugman, "How iris recognition," in IEEE Trans. CVSVT, 2004, pp. 21-30.

[40] J. G. Daugman, "Complete discrete 2-d gabor transforms by neural networks for image analysis and compression," IEEE Transactions on Acoustics, Speech and Signal Processing, vol. 36, no. 7, pp. 1169-1179, 1988.

[41] J. Daugman, "Biometric personal identification system based on iris analysis," U.S. Patent 5291560 , March 01, 1994.

[42] R. C. Gonzalez, R. E. Woods, and S. L. Eddins, Digital Image Processing using MATLAB ${ }^{\circledR}$. Upper Saddle River, New Jersey, USA: Pearson Prentice Hall, 2004, p. 466.

[43] T. Ojala, M. Pietikäinen, and T. Mäenpää, "Multiresolution gray-scale and rotation invariant texture classification with local binary patterns," IEEE Transactions on Pattern Analysis and Machine Intelligence, vol. 24, no. 7, pp. 971-987, 2002.

[44] M. Topi, O. Timo, P. Matti, and S. Maricor, "Robust texture classification by subsets of local binary patterns," International Conference of Pattern Recognition, vol. 03, 2000.

[45] E. Krichen, M. Chenafa, S. Garcia-Salicetti, and B. Dorizzi, "Color-based iris verification," in Advances in Biometrics, D. Zhang and A. K. Jain, Eds. Springer Berlin / Heidelberg, 2007.

[46] N. Muller, "Face recognition using eigenfaces and the crcb colour space," in Proceedings of Pattern Recognition Association of South Africe (PRASA), 2004.

[47] M. Rodriquez, "A graphic arts perspective on rgb-to-cmyk conversion," International Conference on Image Processing, vol. 2, pp. 319-322, 1995.

[48] P.-F. Zhang, D.-S. Li, and Q. Wang, "A novel iris recognition method based on feature fusion," in Proceedings of the Third Internation Conference on Machine Learning and Cybernetics, Shanghai, China, 2004, pp. 3661-3665. 
[49] H. Proença and L. A. Alexandre, "Ubiris:a noisy iris image database," in Proceedings of 13th International Conference on Image Analysis and Processing, vol. 1, 2005, pp. 970-977.

[50] A. Ross and S. Shah, "Segmenting non-ideal irises using geodesoc active contours," in Proceedings of Biometric Symposium (BSYM), Baltimore, USA, 2006.

[51] E. R. Kandel, J. H. Schwartz, and T. M. Jessell, Principles of Neural Science. New York, New York, USA: McGraw-Hill, 2000, pp. 577-580.

[52] H. Proença and L. A. Alexandre, "Toward noncooperative iris recognition: A classification approach using multiple signatures," IEEE Trans. Pattern Anal. Mach. Intell., vol. 29, no. 4, pp. 607-612, 2007.

[53] H. Proença and L. A. Alexandre, "Local intensity variation analysis for iris recognition," The Journal of the Pattern Recognition Society, vol. 37, no. 1, pp. 1287-1298, 2004.

[54] G. A. Carter and D. McCain, "Relationship of leaf spectral reflectance to chloroplast water content determined using NMR microscopy," Remote Sensing of Environment, vol. 46, no. 3, pp. 305-310, 1993. 
Appendix A

Appendix

A.1 Multispectral Iris Data Collection Consent Form 


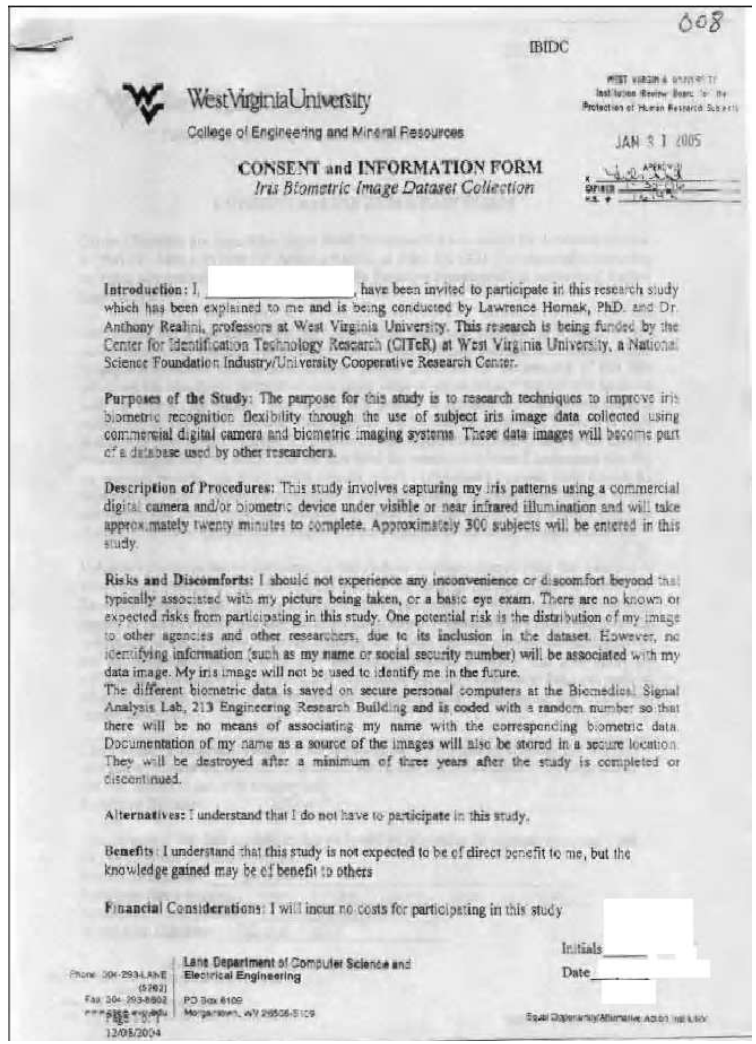




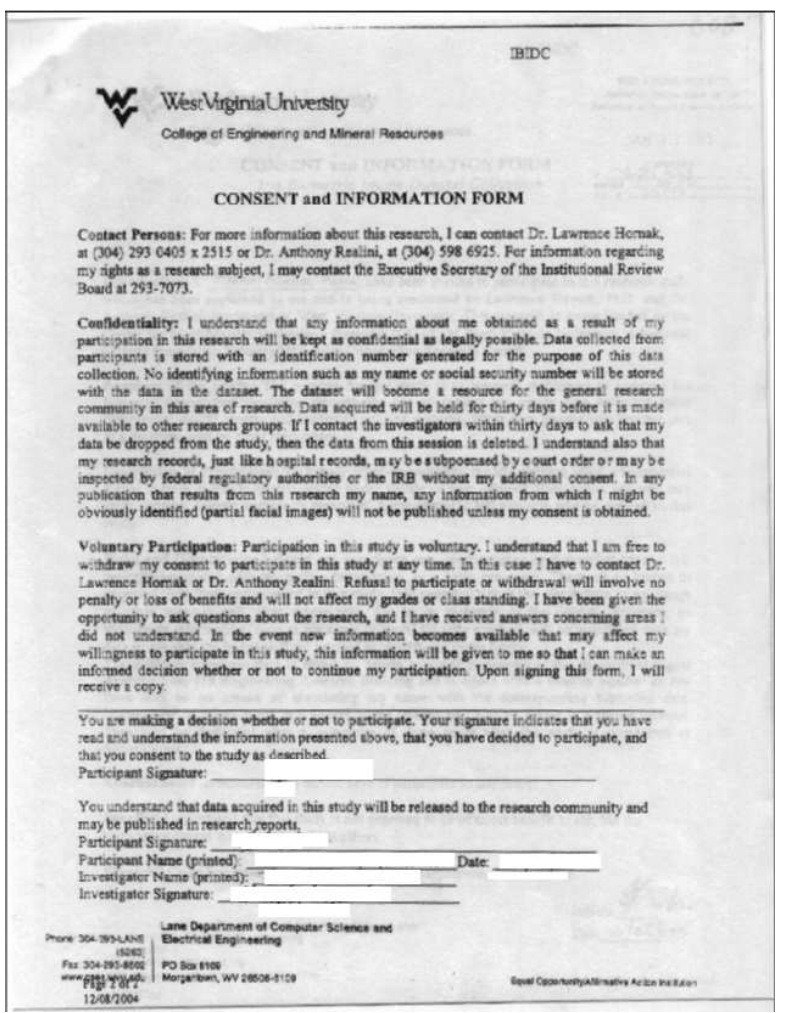

